\title{
Next-Generation Re-Entry Aerothermodynamic Modeling of Space Debris Using Machine Learning
}

Nicholas Sia

West Virginia University, nisia@mix.wvu.edu

Follow this and additional works at: https://researchrepository.wvu.edu/etd

Part of the Aerodynamics and Fluid Mechanics Commons, Astrodynamics Commons, and the Other Aerospace Engineering Commons

\section{Recommended Citation}

Sia, Nicholas, "Next-Generation Re-Entry Aerothermodynamic Modeling of Space Debris Using Machine Learning" (2021). Graduate Theses, Dissertations, and Problem Reports. 8300.

https://researchrepository.wvu.edu/etd/8300

This Thesis is protected by copyright and/or related rights. It has been brought to you by the The Research Repository @ WVU with permission from the rights-holder(s). You are free to use this Thesis in any way that is permitted by the copyright and related rights legislation that applies to your use. For other uses you must obtain permission from the rights-holder(s) directly, unless additional rights are indicated by a Creative Commons license in the record and/ or on the work itself. This Thesis has been accepted for inclusion in WVU Graduate Theses, Dissertations, and Problem Reports collection by an authorized administrator of The Research Repository @ WVU. For more information, please contact researchrepository@mail.wvu.edu. 


\title{
Next-Generation Re-Entry Aerothermodynamic Modeling of Space Debris Using Machine Learning
}

\author{
Nicholas Sia \\ Thesis submitted to the \\ Benjamin M.Statler College of Engineering and Mineral Resources \\ at West Virginia University \\ in partial fulfillment of the requirements for the degree of \\ Master of Science \\ in \\ Aerospace Engineering
}

Piyush M. Mehta, Ph.D, Chair

Jason Gross, Ph.D

$\mathrm{Yu} \mathrm{Gu,} \mathrm{Ph.D}$

Morgantown, West Virginia

2021

Keywords: Re-entry, Aerothermodynamics, Space Debris, Deep Learning Copyright 2021, Nicholas Sia 


\title{
Abstract \\ Next-Generation Re-Entry Aerothermodynamic Modeling of Space Debris Using Machine Learning
}

\author{
Nicholas Sia
}

The number of resident space objects re-entering the atmosphere is expected to rise with increased space activity over recent years and future projections. Predicting the survival and impact location of the medium to large sized re-entering objects becomes important as they can cause on ground casualties and damage to property. Uncertainties associated with the re-entry process makes necessary a probabilistic approach, which can be computationally expensive when using high-fidelity numerical methods for estimating aerothermodynamic properties. To date, object-oriented analysis is the dominant tool used for atmospheric reentry modeling and simulation, where aerothermodynamic coefficients are used to determine the risk a re-entering object poses to the ground through the use of analytical formulations. Closed form solutions are limited to convex objects in the free molecular and continuum flow regime as well as stagnation point estimates. In the transition regime $(75-150 \mathrm{~km})$, a combination of bridging and shape functions are used for the different primitive objects. In this work, the power of deep learning is used to develop next-generation models for the aerothermodynamic modeling (drag coefficient and full body heating distributions) in the transition flow regime for both convex and concave primitive shapes (sphere, cube, and cylinder). The increasing Low-Earth Orbit population puts more stress on NASA's recommended allowable ground risks and makes this a timely contribution. 


\section{Dedication}

For my recently-passed grandfather, who showed us that a little hard work and elbow grease can get you pretty far in life. 


\section{Acknowledgments}

First and foremost, this would not have been possible without the love and support of my parents, whose unending sacrifices as immigrants allowed me to not only pursue a college education, but also a graduate degree and my passion for scientific research.

I am deeply grateful for my graduate advisor and thesis chair, Dr. Piyush Mehta for the tough questions, continuous guidance, and pushing me to be a better scientist each day. This gratitude extends to my graduate committee members, Dr. Jason Gross and Dr. $\mathrm{Yu} \mathrm{Gu}$ for the insightful comments and guidance towards completing my project.

NASA's West Virginia Space Grant Consortium provided the funding for this research under NASA Agreement \#80NSSC20M0055. Furthermore, the High Performance Computing Resources Thorny Flat at West Virginia University (housed at the Pittsburgh Supercomputing Center, funded by the National Science Foundation (NSF) Major Research Instrumentation Program (MRI) Award \#1726534) was used extensively for this work.

Finally, a big debt of gratitude is owed to my cohort members and friends Logan, Aly, Freddy, Joey, Paul, Nate, Benji, Sara, and Dana for the limitless amounts of help and friendship throughout the years. 


\section{Contents}

List of Figures $\quad$ viii

List of Tables

1 Introduction $\quad 1$

1.1 Motivation . . . . . . . . . . . . . . . . . 1

1.2 Objective of Study . . . . . . . . . . . . . . . . . 3

2 Literature Review and Background $\quad 5$

2.1 Object Oriented Analysis . . . . . . . . . . . . . . . . 6

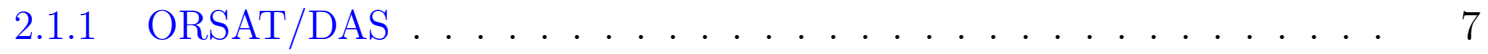

2.1 .2 DEBRISK . . . . . . . . . . . . . . . . 9

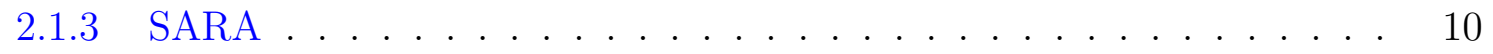

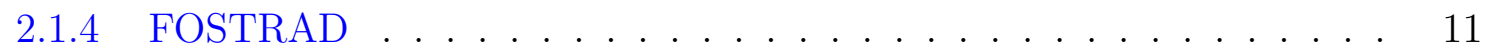

2.2 Spacecraft Oriented Analysis . . . . . . . . . . . . . . . . . 11

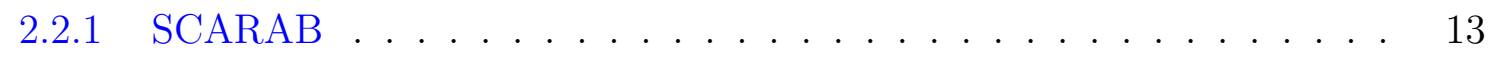

2.2 .2 FAST/MUSIC . . . . . . . . . . . . . . . 14

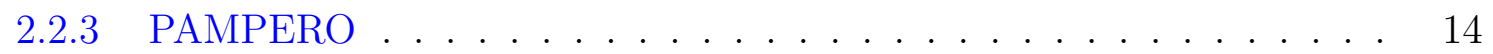

2.3 Analytical Models ... . . . . . . . . . . . . . . 15 
2.3.1 Aerodynamic Modeling . . . . . . . . . . . . . . . . . . . . . . 16

2.3 .2 Thermal Modeling . . . . . . . . . . . . . . . . . . . 17

2.4 Direct Simulation Monte Carlo . . . . . . . . . . . . . . . . . . . . . . . . 21

$\begin{array}{lll}3 & \text { Methodology } & 27\end{array}$

3.1 Geometries and 3D Meshing . . . . . . . . . . . . . . . . . . . 27

3.2 Simulation Database . . . . . . . . . . . . . . . . . . . . . . . . . 29

3.3 Data Processing . . . . . . . . . . . . . . . . . . . . . . . . 33

3.4 Machine Learning . . . . . . . . . . . . . . . . . . . . . . . . . . 34

3.4.1 Gaussian Process Regression Modeling . . . . . . . . . . . . . . . . 34

3.4.2 Artifical Neural Networks . . . . . . . . . . . . . . . . . . . . 35

4 Results $\quad 39$

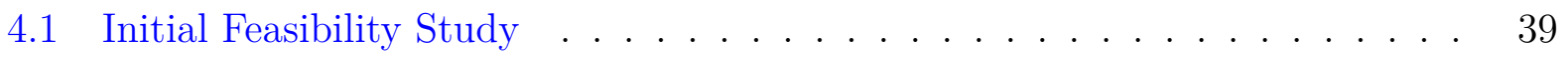

4.2 Drag Coefficient - 2-4km/s Velocity Range . . . . . . . . . . . . . . . . 41

4.3 Drag Coefficient - 6-8km/s Velocity Range . . . . . . . . . . . . . . . . 43

4.4 Heating Distributions - 2-4km/s Velocity Range . . . . . . . . . . . . . 43

4.5 Heating Distributions - 6-8km/s Velocity Range . . . . . . . . . . . . 56

4.6 Scaling Demonstration . . . . . . . . . . . . . . . . . . . 67

4.7 Tumbling Object Demonstration $\ldots \ldots \ldots$. . . . . . . . . . 68

5 Summary, Conclusions, and Suggested Future Work 72 
Bibliography 


\section{List of Figures}

2.1 Re-entry Path $[7] \ldots \ldots \ldots \ldots \ldots \ldots \ldots$

2.2 Object Oriented Simulation Process . . . . . . . . . . . . . . . . 8

2.3 Spacecraft Oriented Simulation Process . . . . . . . . . . . . . . . . 13

2.4 Flow Regimes with Corresponding Knudsen Numbers . . . . . . . . . . . . . 15

2.5 Comparison of Heating Distributions on Convex and non-Convex Shapes . . 20

2.6 Coordinate Definitions for Surface Model for Heating Distributions [12] . . 20

2.7 DSMC Simulation Process . . . . . . . . . . . . . . . . . . . . 26

3.1 Flow Directions for Shape Primitives . . . . . . . . . . . . . . . . . . 29

3.2 Feasibility Study Input Distribution . . . . . . . . . . . . . . . . 31

3.3 Neural Architecture Search Process . . . . . . . . . . . . . . . . . . . . 37

4.1 Sphere Heating Distribution . . . . . . . . . . . . . . . . . . . 40

4.2 Sphere Error . . . . . . . . . . . . . . . . . . . . . . 40

4.3 Sphere Low Heating Case . . . . . . . . . . . . . . . . . . . . . 40

4.4 Sphere High Heating Case . . . . . . . . . . . . . . . . . . . . . . 41

4.5 Drag Coefficient Results for Each Shape . . . . . . . . . . . . . . . . . 42

4.6 Drag Coefficient Results for Each Shape . . . . . . . . . . . . . . . . . 44 
4.7 Sphere Heating Distribution . . . . . . . . . . . . . . . . . . . . . . . . . 46

4.8 Sphere Error . . . . . . . . . . . . . . . . . . . . . . . . . . . . . 46

4.9 Sphere Low Heating Case . . . . . . . . . . . . . . . . . . . . . 47

4.10 Sphere Bridging Function Comparison - Low Heating Case . . . . . . . . . . 47

4.11 Sphere High Heating Case . . . . . . . . . . . . . . . . . . . . . 48

4.12 Sphere Bridging Function Comparison - High Heating Case . . . . . . . . . . 48

4.13 Cube Heating Distribution . . . . . . . . . . . . . . . . . . . . . . 49

4.14 Cube Error . . . . . . . . . . . . . . . . . . . . . . . . . 49

4.15 Cube Low Heating Case . . . . . . . . . . . . . . . . . 50

4.16 Cube High Heating Case . . . . . . . . . . . . . . . . . . . . 51

4.17 Cylinder Side Face Heating Distribution . . . . . . . . . . . . . . . 51

4.18 Cylinder Side Face Error . . . . . . . . . . . . . . . . . . . . . . 52

4.19 Cylinder Side Face Low Heating Case . . . . . . . . . . . . . . . . . . . . 52

4.20 Cylinder Side Face High Heating Case . . . . . . . . . . . . . . . . . 53

4.21 Cylinder Ram Face Heating Distribution . . . . . . . . . . . . . . . 53

4.22 Cylinder Ram Face Error _ . . . . . . . . . . . . . . . . . . . . . . 54

4.23 Cylinder Ram Face Low Heating Case . . . . . . . . . . . . . . . . . . 54

4.24 Cylinder Ram Face High Heating Case . . . . . . . . . . . . . . . . . . . . 55

4.25 Sphere Heating Distribution . . . . . . . . . . . . . . . . . . . . 57

4.26 Sphere Error . . . . . . . . . . . . . . . . . . . . . 57 
4.27 Sphere Low Heating Case . . . . . . . . . . . . . . . . . . . . 58

4.28 Sphere Bridging Function Comparison - Low Heating Case . . . . . . . . . 58

4.29 Sphere High Heating Case . . . . . . . . . . . . . . . . . . . . . . . . . 59

4.30 Sphere Bridging Function Comparison - High Heating Case . . . . . . . . . . 59

4.31 Cube Heating Distribution . . . . . . . . . . . . . . . . . . . 60

4.32 Cube Error . . . . . . . . . . . . . . . . . . . . . . 60

4.33 Cube Low Heating Case . . . . . . . . . . . . . . . . . . . . . 61

4.34 Cube Low Heating Case . . . . . . . . . . . . . . . . . . . . . . 62

4.35 Cylinder Side Face Heating Distribution . . . . . . . . . . . . . . 62

4.36 Cylinder Side Face Error . . . . . . . . . . . . . . . . . . 63

4.37 Cylinder Side Face Low Heating Case . . . . . . . . . . . . . . . . . . . 63

4.38 Cylinder Side Face High Heating Case . . . . . . . . . . . . . . . . . . 64

4.39 Cylinder Ram Face Heating Distribution . . . . . . . . . . . . . . . . 64

4.40 Cylinder Ram Face Error _. . . . . . . . . . . . . . . . . . . 65

4.41 Cylinder Ram Face Low Heating Case . . . . . . . . . . . . . . . . . . 65

4.42 Cylinder Ram Face High Heating Case . . . . . . . . . . . . . . . . . 66

4.43 Scaling Heating . . . . . . . . . . . . . . . . . . . . . 67

4.44 Scaling Heating Distribution . . . . . . . . . . . . . . . . . . . . . . 68

4.45 Cube Heating . . . . . . . . . . . . . . . . . . . . . . . . 69

4.46 Cube Attitude Error . . . . . . . . . . . . . . . . . . . . . . . . . 69 
4.47 Best Training Case . . . . . . . . . . . . . . . . . . . . 70

4.48 Worst Training Case . . . . . . . . . . . . . . . . . . . . . . . 70

4.49 Best Validation Case . . . . . . . . . . . . . . . . . . . . . . . 70

4.50 Worst Validation Case . . . . . . . . . . . . . . . . . . . 71 


\section{List of Tables}

2.1 Shapes Available in ORSAT . . . . . . . . . . . . . . . . . . 9

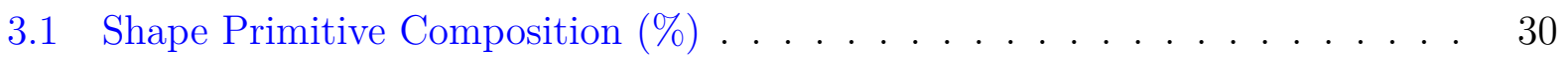

$4.1 \quad$ Drag Coefficient Errors $(\%) \quad \ldots \ldots \ldots . \ldots \ldots 4 \ldots$

4.2 Drag Coefficient Errors $(\%) \ldots \ldots \ldots \ldots$

4.3 Neural Network Training Performance - 2-4km/s Velocity Range . . . . . . . 45

4.4 Neural Network Validation Performance - 2-4km/s Velocity Range . . . . . . 45

4.5 Neural Network Training Performance - 6-8km/s Velocity Range . . . . . . . 56

4.6 Neural Network Validation Performance - 6-8km/s Velocity Range . . . . . . 56

4.7 Neural Network Performance - Attitude Variations . . . . . . . . . . . . 68 


\title{
List of Abbreviations
}

\author{
$\alpha \quad$ Yaw \\ $\alpha_{t} \quad$ Thermal Accommodation Coefficient \\ $\beta \quad$ Pitch \\ $\gamma \quad$ Roll \\ $\gamma \quad$ Specific Heat Ratio \\ $\lambda \quad$ Mean Free Path \\ $\mu \quad$ Mean \\ $\mu_{d} \quad$ Dynamic Viscosity \\ l Matern Parameter \\ $\rho \quad$ Density \\ $\sigma \quad$ Standard Deviation \\ $\sigma_{c} \quad$ Collision Area \\ $\theta \quad$ Flow Inclination Angle \\ A Area \\ $C_{\tau} \quad$ Shear Coefficient \\ $C_{D} \quad$ Drag Coefficient
}




\begin{tabular}{|c|c|}
\hline$C_{h}$ & Heating Coefficient \\
\hline$C_{P}$ & Pressure Coefficient \\
\hline$c_{r}$ & Relative Velocity \\
\hline$D$ & Drag Force \\
\hline$d$ & Kinetic Diameter \\
\hline$E$ & Energy \\
\hline$F_{N}$ & Macroparticle Weight \\
\hline$f_{\text {num }}$ & Number Density \\
\hline$h$ & Specific Enthalpy \\
\hline$k$ & Kernel Parameter \\
\hline$K_{n}$ & Knudsen Number \\
\hline$k_{p}$ & Planetary Constant \\
\hline$L$ & Characteristic Length \\
\hline$N$ & Particle Count \\
\hline$N_{A}$ & Avogadro's Number \\
\hline$P$ & Pressure \\
\hline$P$ & Probability \\
\hline$Q$ & Convective Heat Flux \\
\hline$R$ & Universal Gas Constant \\
\hline
\end{tabular}




\author{
$R_{e} \quad$ Reynold's Number \\ $r_{N} \quad$ Nose Radius \\ $s \quad$ Speed Ratio \\ $S_{t} \quad$ Stanton Number \\ T Temperature \\ $t \quad$ time \\ V Velocity \\ CFD Computational Fluid Dynamics \\ CNES Centre national d'études Spatiales \\ CNN Convolutional Neural Network \\ DEBRISK Debris Risk
}

DRAMA Debris Risk Assessment and Mitigation Analysis

DSMC Direct Simulation Monte Carlo

ESA European Space Agency

FAST Fast Aerothermodynamic Solver for Trans-atmospheric vehicle

FOSTRAD Free Open Source Tool for Re-entry of Asteroids and Debris

GNC Guidance, Navigation \& Control

LEO Low Earth Orbit

MUSIC Multi Simulator in Combination 
NAS Neural Architecture Search

NASA National Aeronautics and Space Administration

NTC No-Time Counter

ODPO Orbital Debris Program Office

ONERA Office National d'Etudes et de Recherches Aérospatiales

ORSAT Object re-entry Survival Analysis Tool

SARA Survival and Risk Analysis

SCARAB Spacecraft Atmospheric Re-Entry and Aerothermal Break-Up 


\section{Chapter 1}

\section{Introduction}

\subsection{Motivation}

Atmospheric re-entry analysis is a critical component to mission planning in order to ensure that the end of a spacecraft's mission is properly planned. Specifically, it is of upmost importance to ensure that any re-entering spacecraft or debris pose minimal threats to civilians, buildings, or populated areas on the ground. As the volume of spacecraft and other space assets in the LEO environment increases, it is expected that space will be considerably more crowded in the next decade and beyond. Per requirements and recommendations outlined by NASA's ODPO to mitigate further pollution of the space environment, satellites are recommended to re-enter Earth's atmosphere within 25 years of the end of its life. Upon re-entry, spacecraft must not pose a greater than 1:10,000 risk of striking any significant objects or causing any civilian casualties on the ground [1].

To date, the predominant analysis tools for re-entry assessment are object-oriented codes such as NASA's ORSAT [2], CNES's DEBRISK [3], and ESA's DRAMA [4]. These tools assume a trajectory in which a spacecraft will fragment into multiple shape primitives at a certain altitude, and the following risk analysis is performed on these simple objects [7]. Conversely, spacecraft-oriented tools such as ESA's SCARAB [8], CNES's PAMPERO [9], ONERA's FAST/MUSIC [10] simulate the entire spacecraft until demise to determine its risk[11]. Both types of tools determine drag and heating coefficients for risk assessment 
through the use of semi-empirical and analytical formulations. For finite nose radius objects such as a sphere, closed form solutions exist for stagnation point heating [12] [13] [14]. The opposite is true for nonconvex shapes such as cubes, cylinders, and other objects with sharp edges. For these geometries, some sort of estimation is achieved with the use of shape factors or bridging functions [7]. These models are quickly evaluated, but offer low fidelity and do not offer the ability to determine the uncertainties associated with the predictions.

High-fidelity numerical tools such as DSMC codes can provide estimates for aerodynamic coefficients and heating distributions given certain flow conditions. The main drawback to these tools is computational expense (where analytical formulations excel): the computational resources needed to numerically solve particle motion and surface interactions scales exponentially with increasing atmospheric density [15].

Re-entry flow is typically classified into one of three flow regimes: Continuum, transition, and free molecular [16]. The bounds of each flow regime are characterized by a specific Knudsen Number, which defines the kinetic distance between gas particles compared to some characteristic length [17]. In free molecular flow, the Knudsen Number is sufficiently high enough such that intermolecular collisions are improbable. In this flow regime, where heating is not much of a concern, it is possible to use high-fidelity techniques such as DSMC with full-scale spacecraft geometries with a relatively small amount computational expense; however, real-time or probabilistic applications are still not feasible with DSMC for freemolecular flow. While transition flow generally corresponds to a denser accumulation of gas particles, DSMC is still valid here, albeit requiring more computational resources; however, in the continuum flow regime, intermolecular collisions are constantly happening due to the density of particles in any flow field. For this reason, DSMC starts to become impractical due to the sheer amount of computational resources required. At altitudes where the flow can be classified in the continuum regime, heating is at its highest and the use of CFD is 
required. For intensive cases such as those relating to the flows in the lower thermosphere (Approximately $90 \mathrm{~km}$ ), simulation times with DSMC vary from a few days to a few weeks, depending on available computational resources. This makes uncertainty quantification and establishing a probabilistic casualty risk area in a practical timeframe highly unlikely.

However, a tradeoff can be reached through machine learning techniques. Given a set sample size of heat flux distributions, a deep learning architecture, such as a fully connected or convolutional neural network, can be trained to produce full-detail solutions on a given set of inputs such as atmospheric state and object attitude. This enables accurate emulation of the numerical solutions for a given set of flow conditions that can be produced almost instantly. With this robust framework, re-entry computations can be completed quickly while maintaining the same fidelity as numerical methods, thereby making possible probabilistic analyses through a large number of ensemble runs.

\subsection{Objective of Study}

The overall proposed goal of this project is to demonstrate the use of modern, open-source deep learning techniques to predict aerothermodynamic responses of shape primitives in re-entry flow. Specifically, it is proposed that these models address:

- Non-uniform aerothermal heating on the surface of non-convex shape primitives

- Elimination of exponentially scaling computational resources needed for low-transition to continuum flows

- The combination of deep learning models with open-source re-entry analysis tools to create a robust framework that can accurately predict heating based on visible area 
- The possibility of uncertainty quantification and likelihood analysis which has been largely impossible

- Creation of transition-regime functions that do not require shape factors or analytical formulations with poorly defined error quantification 


\section{Chapter 2}

\section{Literature Review and Background}

As mentioned previously, three avenues exist for aerothermodyanmic modeling: objectoriented tools, spacecraft-oriented tools, and numerical simulations. Object oriented tools perform analysis on critical parts (modeled as simple shapes) of the re-entering spacecraft that may survive the initial demise event whereas spacecraft-oriented tools analyze the entire spacecraft from the re-entry sequence to the demise and subsequent risk analysis of surviving objects. Both these tools make use of analytical models for drag and heating coeffcient which reduces computational load; however, these tools are low fidelity and only closed-form solutions exist in the free molecular and continuum flow regimes for convex objects. Otherwise, bridging functions and fudge factors are used in the transition flow regime and for other shapes [7]. Numerical codes such as DSMC remain the gold standard as they are generally considered to provide the highest-fidelity results in producing drag and heating assessments. Computational expense is currently the main barrier preventing DSMC from widespread use, thus the need for a more efficient high-fidelity model is born.

Currently, the main issue that still exists within aerothermodynamic modeling is the computational expense required. Simulation methodologies such as DSMC, which stochastically models gas particle collisions to simulate rarefied gas flows, require the use of highperformance computing clusters [15]. Specifically, in areas of the atmosphere that fall within the free molecular regime, the atmospheric density is sparse enough that the simulation grid is not fine enough to require large simulation times. Significant efforts have led to notewor- 
thy improvements in our ability to modeling heating distributions; however, a lot remains to be achieved, especially for non-convex objects such as cubes and cylinders [12]. These numerical methods can be accurate but require a significant amount of computing resources. Work by Mehta et. al provides a machine learning model for satellite drag coefficients [19]. A similar framework can be used, but will need to be expanded to be able to produce a full spatial distribution over the object rather than a singular value.

\subsection{Object Oriented Analysis}

Object oriented tools perform aerothermodynamic analysis on components of a spacecraft with shape primitives as an approximation of these parts [7]. A key component to object oriented analysis is an assumption of a certain demise altitude (usually $75 \mathrm{~km}$ ), in which the spacecraft is expected to decompose into individual objects with shape primitives. Due to this decomposition, a simplifying assumption is made where only critical elements of the spacecraft need to be analyzed as compared to the entire body reducing the computational complexity of the simulation. For each critical part, aerothermodynamic analysis is performed, and if the heat experienced by the object exceeds the critical heating, then the object will demise. If not, the object will survive the heat of atmospheric re-entry. Figure 2.1 shows a typical re-entry path, where object oriented analysis is conducted after the initial breakup.

Typically at the start of analysis, the appropriate shape primitives are chosen to represent critical parts of the spacecraft. With each shape, thermodynamic properties such as mass and material are assigned for the simulation. Then, these objects are propagated along their respective re-entry paths to determine survivability for each component. This is typically determined by comparing the thermal load experienced versus the heat of ablation. 


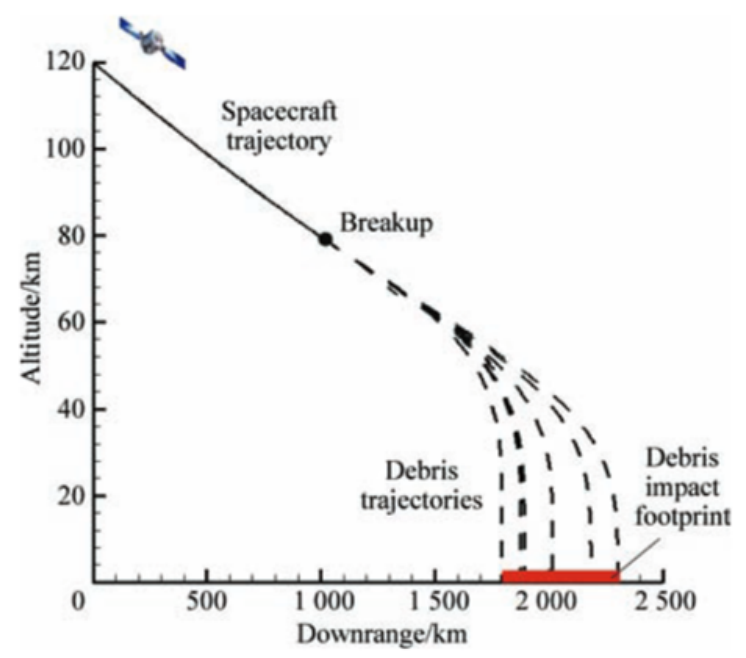

Figure 2.1: Re-entry Path [7]

If an object survives (thermal load is not greater than heat of ablation), a ground impact footprint (risk area) for that object is assessed. If not (thermal load is greater than heat of ablation), the object is considered to demise completely and not cause any threats to assets on the ground. This process is repeated for each object until the simulation is complete. Figure 2.2 summarizes this approach.

\subsubsection{ORSAT/DAS}

The most popular object oriented tool available is NASA's ORSAT, which includes four different shape primitives: sphere, cylinder, flat plate and a cube [7]. In the propagation of these objects downrange, a 3-DOF ballistic model is commonly used. Attitude dynamics are not directly solved for tumbling objects but are instead included as predefined configuration as shown in Table 2.1. For non-tumbling objects, aerothermodynamic properties are computed analytically; however, much of the actual formulations used in ORSAT are proprietary and are not available to the public. For tumbling objects, shape factors are used in conjunction with the analytical predictions for non-tumbling shapes [21]. For ablation mod- 


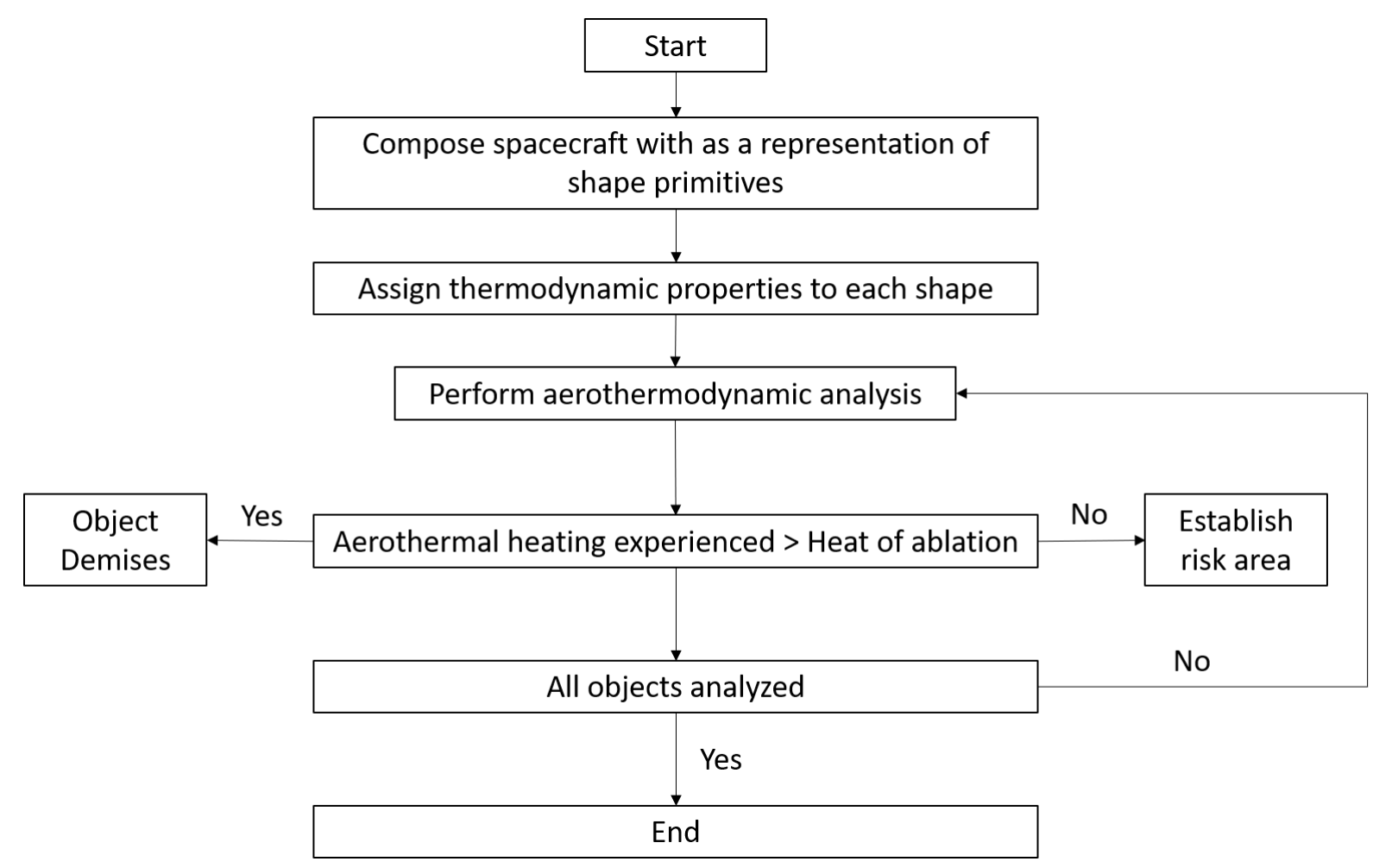

Figure 2.2: Object Oriented Simulation Process

eling, ORSAT considers two approaches: the lumped mass (zero-dimensional) approach, and the one-dimensional heat conduction approach [20]. The lumped mass approach does not consider temperature distributions or melting rates of an object. This allows the simplification of predicting ablation as it treats each object as a singular node. Conversely, the heat conduction approach solves the heat conduction equation allowing object partial melting to be modeled.

Another popular tool used by NASA is the Debris Assessment Software (DAS). Meant to be a quick-to-evaluate tool, DAS functions in a very similar way to ORSAT in its evaluation of debris risk. The main output of a full re-entry risk assessment with DAS is the risk area projected by the surviving debris. The total casualty area is calculated as: 
Table 2.1: Shapes Available in ORSAT

\begin{tabular}{cc}
\hline Shape & Configurations \\
\hline Sphere & Spinning \\
Not Spinning & \\
Cube & Head-on flow \\
Tumbling & \\
Cylinder & Broadside, Spinning \\
& Random Tumbling and Spinning \\
& Ram-Face and Spinning \\
& End-over-end Tumbling and Spinning \\
Flat Plate & Head-on flow \\
& Tumbling \\
\hline
\end{tabular}

$$
A_{c}=\sum_{i}\left(0.6+\sqrt{A_{i}}\right)^{2}
$$

Under this formulation, the casualty area as outlined by NASA safety standards should not exceed $8 m^{2}$ [1]. DAS is meant to be used as a first risk assessment; according to NASA safety standards, if the casualty area is not acceptable, more accurate methods should be used (ORSAT).

\subsubsection{DEBRISK}

Aside from ORSAT/DAS, object oriented tools developed by other countries/agencies exist. One is DEBRISK created by CNES, which was developed in response to an act from French 
Parliament dictating that satellite manufacturers must consider possible risks posed from end-of-life operations for reentering space assets [22]. DEBRISK functions much of the same way other object oriented tools do, implementing very similar engineering models akin to those used in ORSAT and other codes. One area in which DEBRISK differs is its ability to dynamically update the size of the object in its ablation modeling. Typically, a check is completed to determine if the aerothermal heating experienced exceeds the threshold for the material and object. If the heating exceeds the critical threshold, then the object demises; but, DEBRISK further models the rate of ablation to dynamically update the mass and size of each object along the propagation of its trajectory before completing the simulation.

Recent work by Annaloro et. al expands DEBRISK's capabilities into aerothermodynamic modeling for complex shapes including hollow shape primitives [3]. Rather than utilize analytical formulations, the update makes use of database of CFD simulations to interpolate the drag force and the convective heat flux based on the definition of the shape. So far, the update is only for the continuum flow regime, as work in the transition and free-molecular flow regimes are subject of ongoing work.

\subsubsection{SARA}

The final commonly used re-entry tool is SARA which was developed by ESA in response to the European Code of Conduct for Space Debris Mitigation [4]. SARA is a piece of software that independently operates as part of ESA's greater re-entry risk tool suite, DRAMA. SARA operates in a very similar fashion to DAS and is meant to be used in conjunction with the other tools available in DRAMA. 


\subsubsection{FOSTRAD}

A recently-developed tool by the University of Strathclyde is FOSTRAD which performs object oriented analysis using the equations shown in Section 2.3 [5] [6]. FOSTRAD makes use of a panel method in which heating distributions are calculated on individual mesh elements that compose a 3D Geometry. To simplify calculations, only mesh elements that are visible to the flow are considered for each point in the objects trajectory. The propagator that models the movement of the object along the re-entry corridor is similar to the 3-DOF model seen in other object oriented tools.

\subsection{Spacecraft Oriented Analysis}

Aside from object-oriented tools, spacecraft-oriented tools exist to provide what is generally considered a more accurate and complete analysis of a spacecraft from the start of the re-entry sequence to the demise of individual components. The main difference comes in what is specifically modeled and analyzed in these simulations: in object-oriented codes, aerothermodynamic properties of individual components of spacecraft are analyzed (in the

form of shape primitives). In spacecraft-oriented codes, the complete spacecraft assembly is modeled as a collection of shape primitives. While this offers a more realistic analysis of a reentering spacecraft, the computational expense is significantly higher due to the detail required to accurately capture the features of the spacecraft. These codes make use of the panel method, similar to what is used in FOSTRAD, where aerothermodynamic properties are a function of the position of individual mesh elements in the 3D model of the desired spacecraft. For example, in modeling the BeppoSAX Satellite for use in spacecraft oriented analysis, 859 primitives were used which translated to 177,708 surface panels and 72,548 volume panels. Computing drag and heating values for each panel at each timestep along 
its trajectory across the re-entry corridor becomes computationally demanding and complex [24] . Noteworthy improvements, specifically the pixelator developed by Mehta et.al [56], can lead to improvements in reducing computational expense of the geometries modeled during analysis but matching the efficiency of object oriented analysis has proved to be difficult.

Despite the increase in complexity and differences in modeling, spacecraft oriented analysis functions much of the same way that object oriented analysis does. Fragments of the spacecraft are analyzed along the re-entry trajectory to determine if the spacecraft completely demises and if not, what portions will survive; however, what makes spacecraft-oriented analysis unique is that the spacecraft is wholly modeled to be as accurate to the overall geometry as possible. Then, along the trajectory, the fragmentation analysis is performed to determine when the demise of the spacecraft will start to occur. Once the fragmentation analysis is complete, each fragment is aerothermodynamically analyzed for demise. These types of codes are much more detailed in their analysis as the entire re-entry process is modeled with minimal assumptions for greater amounts of accuracy. In object-oriented tools, a breakup altitude is assumed and each primitive is analyzed according to a 3-DOF model. In spacecraft-oriented modeling, a breakup altitude is determined by dynamically calculating the thermal and mechanical loading on the spacecraft along the re-entry trajectory. In modeling the trajectory, a 6-DOF model is used to include the attitude of the spacecraft during the simulation. Furthermore, the thermal and mechanical load calculations utilize a local approach and determine aerothermodynamic properties for each mesh element of the spacecraft model. Figure 2.3 summarizes the simulation methodology taken.

To date, three spacecraft-oriented codes are known to exist: [25]

- SCARAB

- FAST/MUSIC 
- PAMPERO

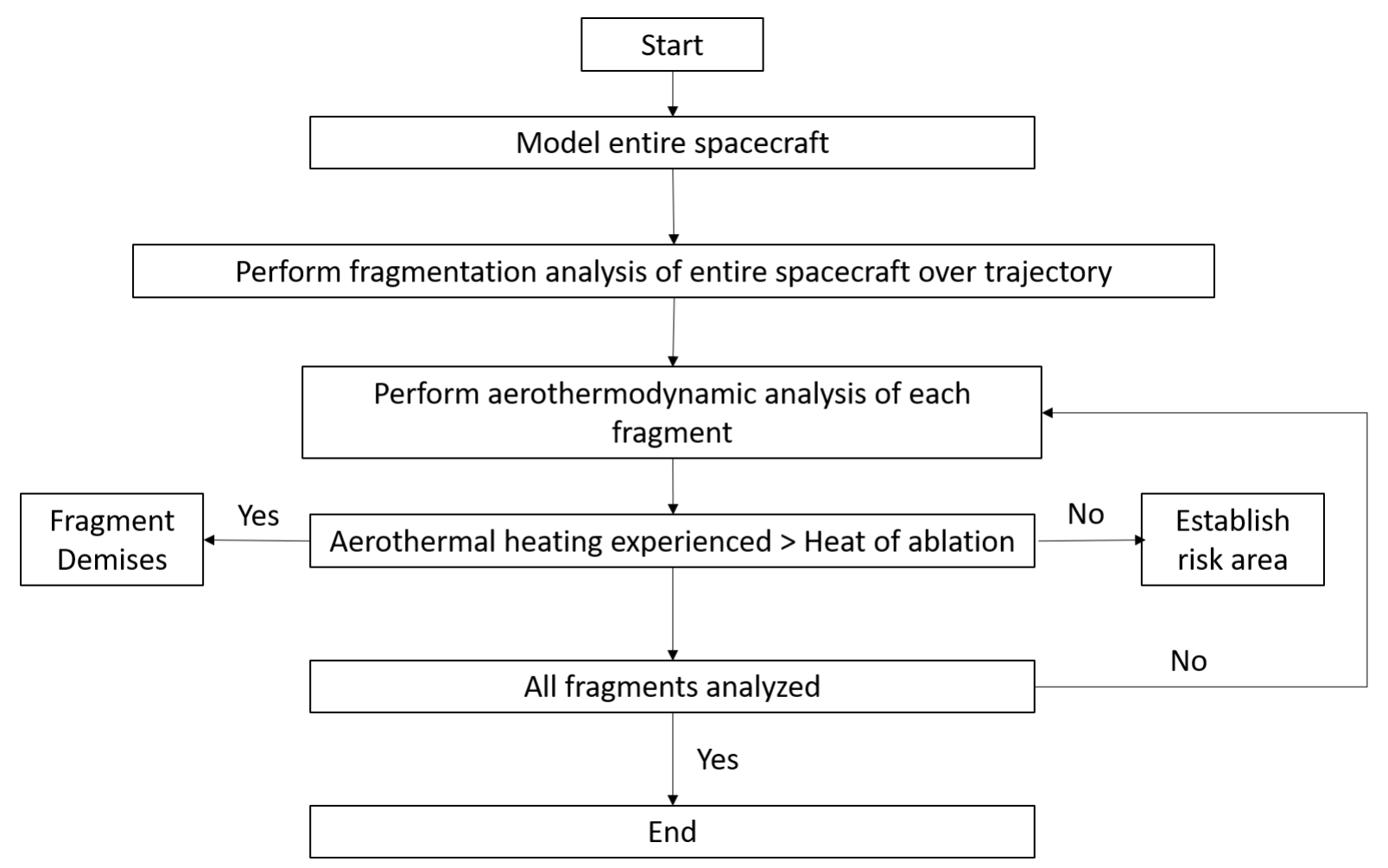

Figure 2.3: Spacecraft Oriented Simulation Process

\subsubsection{SCARAB}

SCARAB is a spacecraft-oriented software developed by ESA that allows researchers to examine the mechanical and thermal destruction of spacecraft and other objects during reentry [8]. It makes use of a 6-DOF model that performs risk assessments. SCARAB has been validated with in-flight measurements, re-entry observations and wind tunnel experiments $[25]$. 


\subsubsection{FAST/MUSIC}

ONERA has been developing 6-DOF FAST/MUSIC since 2006 to compute the atmospheric re-entry of controlled and uncontrolled objects such as space debris, asteroids, and vehicles (multi-object modeling is allowed). The GNC code MUSIC is combined with the aerothermodynamic code FAST [23]. The trajectory of fragments is calculated using a Runge-Kutta scheme or a fourth order predictor-corrector system to numerically integrate the 3 or 6 degrees of freedom equations of motion. FAST/MUSIC has been compared to experimental data, numerical (CFD/DSMC) data, as well as other spacecraft-oriented codes for a variety of geometric shapes encountered.

\subsubsection{PAMPERO}

PAMPERO is a spacecraft-oriented tool developed by CNES in 2013. The conventional Runge-Kutta method is used to integrate the object's trajectory and attitude (6-DOF) [9]. For each of the three flow regimes, local pressure and friction coefficients may be computed (i.e free molecular, transitional and continuum). Analytical formulas are used to predict the convective heat flux at the stagnation point with correlations generated from CFD/DSMC simulations. The convective heat flux computation is done all around the object using an empirical function that is based on the curvature radius and local pressure. In order to accommodate the transitional regime, bridging functions are required. The losses of radiant heat fluxes from the wall item are also calculated; however, the shock layer's radiation is not considered. The diffusion heat fluxes on the surface and inside the object are calculated using a 3D thermal module. Additionally, an ablation model has been implemented. 


\subsection{Analytical Models}

As mentioned above, analytical formulations are used in aerothermodynamic analysis within both spacecraft-oriented and object-oriented codes. Analytical re-entry models offer advantages over numerical simulations in the fact that they are extremely fast to evaluate; however, the downfall comes in the existence of closed form solutions. As mentioned above, re-entry flow is characterized in three different regimes: free-molecular, transition, and continuum flow. The bounds for each regime is dictated by a dimensionless parameter known as the Knudsen Number, calculated as:

$$
K_{n}=\frac{\lambda}{L}
$$

Where,

$$
\lambda=\frac{R T}{\sqrt{2} \pi d^{2} N_{A} P}
$$

Free molecular flow corresponds to portions of the atmosphere where $K_{n}>1$, continuum flow corresponds to $K_{n}<0.01$, and transition flow refers to the region whose Knudsen Numbers fall in between. There are no hard boundaries in defining flow regimes as these are typically assumed. Figure 2.4 shows the assumed bounds for each flow regime, highlighting the regime of focus for this work.

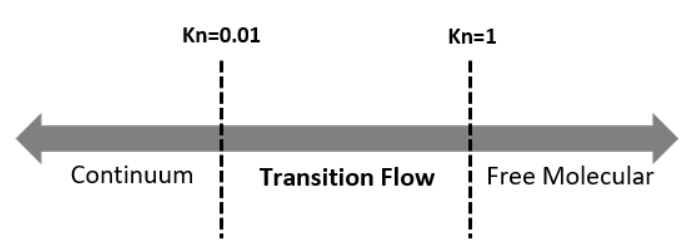

Figure 2.4: Flow Regimes with Corresponding Knudsen Numbers 


\subsubsection{Aerodynamic Modeling}

For all shapes, closed form equations for drag exist in the free molecular and continuum flow regime as a function of the shear and pressure coefficients. In free molecular flow, the aerodynamic contribution (drag coefficient) is developed from Schaaf and Chambre's analytical model [26].

$$
\begin{gathered}
C_{P}=\frac{1}{s^{2}}\left[\left(\frac{2-\sigma_{N}}{\sqrt{\pi}} s \sin \theta+\frac{\sigma_{N}}{2} \sqrt{\frac{T_{w}}{T_{\infty}}}\right) e^{-(s \sin \theta)^{2}}+\right. \\
\left.\left\{\left(2-\sigma_{N}\right)\left((s \sin \theta)^{2}+\frac{1}{2}\right)+\frac{\sigma_{N}}{2} \sqrt{\frac{\pi T_{w}}{T_{\infty}}} s \sin \theta\right\}(1+\operatorname{erf}(s \sin \theta))\right] \\
C_{\tau}=-\frac{\sigma_{T} \cos \theta}{s \sqrt{\pi}}\left[e^{-\left(s \sin \theta^{2}\right)}+\sqrt{\pi} s \sin \theta(1+\operatorname{erf}(s \sin \theta)]\right. \\
C_{D}=C_{P}+C_{\tau}
\end{gathered}
$$

For aerodynamics in the continuum regime, Modified Newtonian Theory is used to determine the pressure coefficient as a function of the local flow inclination angle [29]:

$$
C_{p}=C_{p, \max } \sin ^{2} \theta
$$

In this regime, the shear contribution is assumed to be negligible, making the drag coefficient equal to the pressure coefficient.

For drag modeling in the transition regime, a bridging function for each force coefficient developed by Wilmoth is used for both pressure and shear contributions [31]: 


$$
C_{X, \text { trans }}=C_{X, c}+\left(C_{X, F M}-C_{X, c}\right) \sin \left(\pi\left(a+b \log _{10}(K n)\right)\right)^{2}
$$

Where $a$ and $b$ correspond to the upper and lower bounds of the transition flow as dictated by Knudsen Number, as such the values of these constants are as follows:

$$
\begin{gathered}
a=1 \\
b=0.01
\end{gathered}
$$

\subsubsection{Thermal Modeling}

In the free molecular and continuum flow regimes, closed form heating solutions for heating only exist for finite-nose radius shapes, such as a sphere. For sharp-edged shapes such as flat plates, cubes, cylinders, etc., no closed form solutions exist. Instead, bridging functions and shape factors are used in attempts to approximate thermodynamic properties of these shapes as a function of existing closed form solutions. For a sphere in Free Molecular flow, the heat transfer on a sphere can be calculated as:

$$
\begin{gathered}
Q=\frac{\alpha_{t} \rho_{\infty} V_{3}}{4 s^{3} \sqrt{\pi}}\left[\left\{s^{2}+\frac{\gamma}{\gamma-1}-\frac{\gamma+1}{2 \gamma-1} \frac{T_{w}}{T_{\infty}}\right\}\right. \\
\left.\left\{e^{-s^{2} \sin ^{2} \theta}+\sqrt{\pi} s \sin \theta[1+\operatorname{erf}(s \sin \theta)]\right\}-\frac{1}{2} e^{-s^{2} \sin ^{2} \theta}\right]
\end{gathered}
$$

Where $s$ is defined as:

$$
s=\frac{V_{\infty}}{\sqrt{2 R T_{\infty}}}
$$

In continuum flow, aerothermal heating can be approximated in one of four ways. 
One is the Detra-Kemp-Riddell formulation which is used in ESA's spacecraft oriented code SCARAB [27].

$$
Q=\frac{10.6 \times 10^{7}}{\sqrt{R_{n}}} \sqrt{\frac{\rho}{\rho_{0}}}\left(\frac{V}{V_{0}}\right)^{3.15}
$$

Fay-Riddell carried out a study accounting for the dissociation effects. Under the assumptions of a laminar boundary layer, equilibrium flow, constant Prandtl number (Pr) value of 0.71 , constant Lewis number (Le) of 1 , and a catalytic wall, the relation is given as [13]:

$$
Q=0.94\left(\rho_{w} \mu_{w}\right)^{0.1}\left(\rho_{s} \mu_{s}\right)^{0.4}\left(h_{s}-h_{w}\right) \sqrt{\left(\frac{d u_{e}}{d x}\right)_{s}}
$$

Where the last term is the velocity gradient at the stagnation point of the wall:

$$
\sqrt{\left(\frac{d u_{e}}{d x}\right)_{s}}=\frac{1}{r_{N}} \sqrt{\frac{2\left(p_{s}-p\right)}{\rho_{s}}}
$$

Van Driest further simplified this equation since properties at the surface of the object are often difficult to approximate [14]:

$$
Q=0.94\left(\rho_{s} \mu_{s}\right)^{0.4}\left(h_{s}-h_{w}\right) \sqrt{\left(\frac{d u_{e}}{d x}\right)_{s}}
$$

Sutton-Graves also developed another formulation for stagnation point heating that can be used on either Mars or Earth, depending on the planetary constant, $k$ [28]:

$$
Q_{s}=k \sqrt{\left(\frac{\rho}{R_{n}}\right)} V^{3}
$$


Where, for Earth's atmosphere:

$$
k_{p}=1.7415 \times 10^{-4}
$$

While closed form solutions for finite nose radius objects exist in the continuum and free-molecular flow regime as shown above, no such solutions exist in the transition regime. Bridging functions have historically been used to approximate heating and drag coefficients in this regime. For heating, a formula developed by Legge which is a function of both free molecular and continuum stagnation heating is used [30]:

$$
Q_{s, \text { trans }}=\frac{Q_{s, \text { cont }}}{\sqrt{1+\left(\frac{Q_{s, \text { cont }}}{Q_{s}, F M}\right)^{2}}}
$$

In each flow regime, the equations presented are only to determine the stagnation point heating for finite nose radius shapes. To produce a whole distribution, a function was developed for use in FOSTRAD that maps a heating surface distribution according to the local flow inclination angle [12]:

$$
Q(\theta)=Q_{s}(0.1+0.9 \cos \theta)
$$

In terms of modeling full heating distributions, Eq. 2.9 and Eq. 2.17 offer closed form solutions as the mapping across the surface is a function of a local flow inclination angle; however, for sharp edged objects, the effects of flow separation due to hypersonic shockwaves must be considered. In curved objects with a defined nose radius, no such separation occurs. For other objects, flow separation causes significantly higher heating at corners and edges causing those portions of space debris to melt and demise first. This is illustrated in Figure 2.5 where the areas of concentrated heating can be seen at the corners and edges of the cube. 

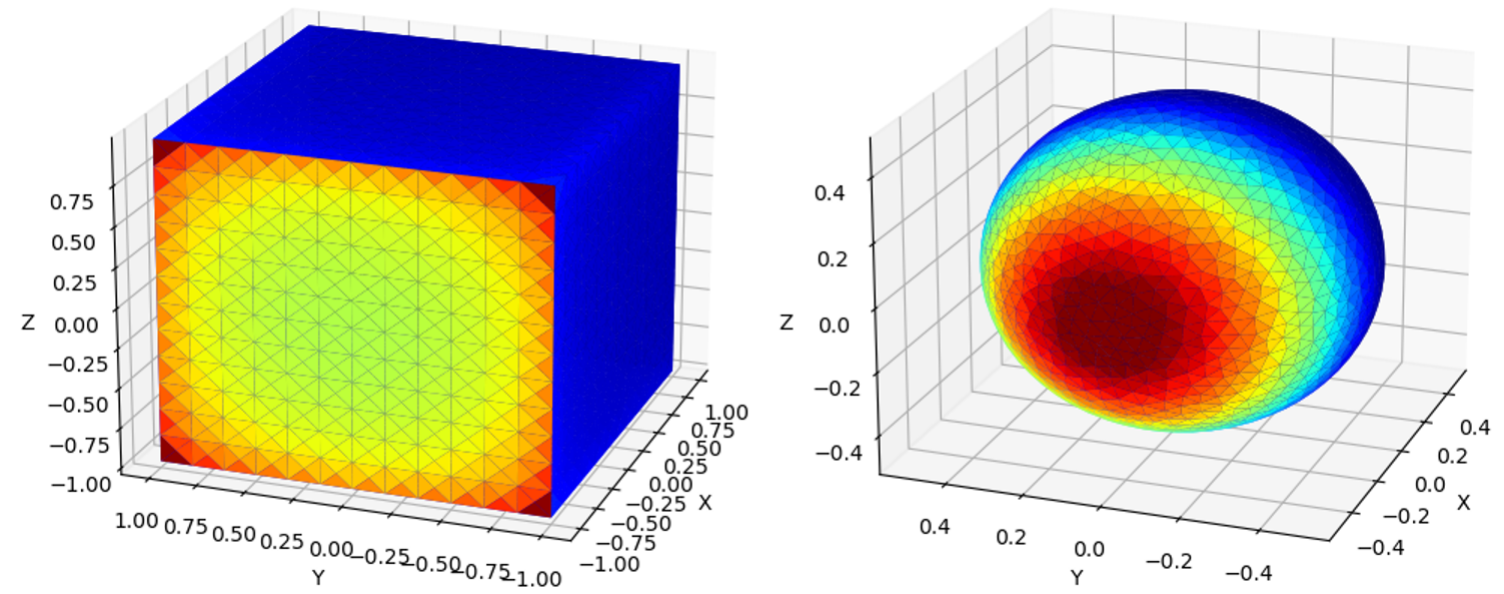

Figure 2.5: Comparison of Heating Distributions on Convex and non-Convex Shapes

Mehta et.al [12] attempted to model the surface distribution on a cube and cylinder assuming no attitude variations are present. Under this simplification along with the assumption that heating is perfectly symmetrical on the face directly impacted by the flow, a simple model is defined as a function of coordinate points on the face of the object shown in Figure 2.6.
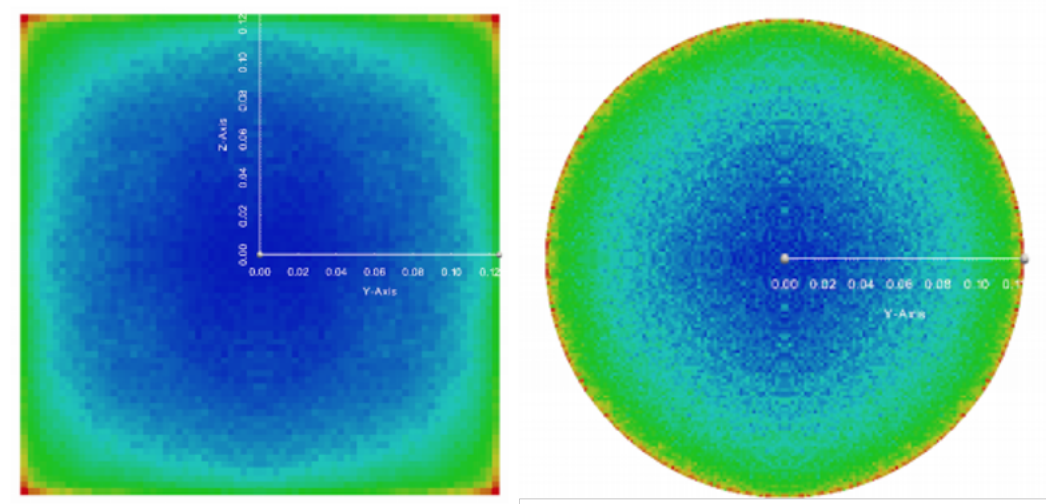

Figure 2.6: Coordinate Definitions for Surface Model for Heating Distributions [12]

The heating at any arbitrary point is then given as: 


$$
C_{h_{Y, Z}}=\sqrt{C_{h, Y}^{2}+C_{h, Z}^{2}}
$$

Where $C_{h}$ is calculated by the following equation

$$
C_{h}=\frac{2 Q}{\rho_{\infty} V_{\infty}^{3}}
$$

\subsection{Direct Simulation Monte Carlo}

DSMC is a numerical technique that is commonly used to model rarefied gas flows for finite Knudsen Numbers. Developed by G. Bird, the technique uses a stochastic simulation to probabilistically solve the Boltzmann Equation and model particle motion and interactions within a given simulation domain [32]. The particles in the simulation are representative of a group of "real" particles (most often defined as a ratio of real-to-simulated particles) and their motions with respective intermolecular, surface, and boundary reactions are modeled as a function of time. Flow properties such as temperature, density, and velocity are sampled from the particle state on a macroscopic scale over a set amount of timesteps [33].

DSMC is unique compared to most commercial CFD tools as it utilizes a Lagrangian approach and models the trajectory of each representative particle in the flow whereas CFD takes a Eularian approach and models flow within a given control volume [35]. Furthermore, as mentioned above, DSMC uses a Monte-Carlo simulation to probabilistically solve the Boltzmann equation as compared to CFD where the Navier Stokes equation is solved by some finite-discretization scheme [36].

Within DSMC, the following parameters must be defined for the simulation: 
- Boundary conditions

- Simulation domain bounds in all three directions (bounding box)

- Cells in each direction

- Flow Density and real-to-simulated particle ratio

- Chemical species present in mixture with respective mole fractions

- Flow temperature and flow velocity

- Geometry to be used in the simulation

- Surface temperature of object

- Timestep size and amount of timesteps for which the simulation spans

The boundary conditions for DSMC simulations pertaining to atmospheric re-entry flow are set to be open, allowing particles to freely exit and enter the entire domain in all directions. As for the bounds, the space containing the geometry must be sufficiently large enough so that pertinent flow phenomena such as the presence of a supersonic shockwave can be completely captured and modeled. The amount of cells in each direction is typically uniform as it is set to be at least half of the mean free path of the mixture:

$$
l<\frac{\lambda}{2}
$$

Within each cell, the amount of particles present must be enough such that flow states can be accurately sampled. It is generally best practice that the amount of particles per cell is anywhere from 5-10, and the ratio of real-to simulated particles is defined as: 


$$
f_{\text {num }}=\frac{n}{2}
$$

To ensure temporal independence, the timestep is defined such that each particle moves through at most a single cell within a given timestep. As such, it is an indirect function of the flow density and velocity:

$$
\Delta t \leq \frac{\lambda}{V_{\infty}}
$$

In turn, the duration of the simulation is set by the user such that the sampling of the flow within the simulation has completely converged without large variation in flow state which is highly dependent on the complexity of the simulation and flow density/velocity.

A summary of the DSMC simulation process can be seen in Figure 2.7. At the beginning of the simulation, the bounding box as well as the individual cells within the domain are first created and indexed. Next, the particles according to the given density are initialized in the cell network with their initial velocities and positions. Then, the particle trajectories are modeled in each timestep as they move through time. Within each timestep, the intermolecular collisions and surface interactions are probabalistically modeled. In a simulation cell, the probability of a collision for a simulated particle is [34]:

$$
P=F_{N}\left(\frac{\sigma_{T} c_{r} \Delta t}{V_{c}}\right)
$$

In highly dense flows where there are a large amount of macroparticles present, calculating this probability becomes extremely demanding and inefficient. As such, the NTC method was developed to determine the amount of macroparticle pairs to check within each 
timestep for the probability of collisions, defined as:

$$
N_{\max }=\frac{N \bar{N} F_{N}\left(\sigma_{T} c_{r}\right)_{\max } \Delta t}{2 V_{c}}
$$

For each pair, the probability of collisions is then:

$$
P=\frac{\sigma_{t} c_{r}}{\left(\sigma_{T} c_{r}\right)_{\max }}
$$

This value is then compared to a random number, $R$, and if $P>R$, a collision occurs. From these collisions, the velocity of each particle after the collision is as follows:

$$
\begin{gathered}
c_{1}^{\star}=c_{m}+\frac{m_{1}}{m_{1}+m_{2}} c_{r}^{\star} \\
c_{2}^{\star}=c_{m}-\frac{m_{1}}{m_{1}+m_{2}} c_{r}^{\star}
\end{gathered}
$$

The deflection angle of the particle can the be calculated:

$$
\cos \theta=2 R_{1}-1
$$

From these interactions, the flow properties are sampled which leads to the output of the variables of interest (in this case, drag and heating acting on the body). Within the DSMC simulation, drag forces acting on a body is calculated by summing the change in momentum of each particle interacting on the surface over each simulation particle: 


$$
D=-\sum_{n=1}^{\# \text { particles }} \frac{m \Delta V f_{\text {num }}}{\Delta t}
$$

From this, the drag coefficient can be calculated:

$$
C_{d}=\frac{2 D}{\rho A V^{2}}
$$

Heat flux acting on the object is determined as the sum of kinetic, rotational and vibrational energies due to the motion of the particle:

$$
\begin{aligned}
& E_{k}=\frac{1}{2} m\left(V_{2}^{2}-V_{1}^{1}\right) \\
& E_{r}=E_{r 2}-E_{r 1} \\
& E_{v}=E_{v 2}-E_{v 1} \\
& \dot{Q}=E_{k}+E_{v}+E_{r}
\end{aligned}
$$

This process is repeated for the set amount of timesteps before the simulation terminates, shown in Figure 2.7.

The DSMC package used in this study is the Stochastic PArallel Rarefied-gas Timeaccurate Analyzer (SPARTA), an open source DSMC code developed by Sandia National Laboratories. SPARTA benefits from being highly adaptable and efficient with options to add 


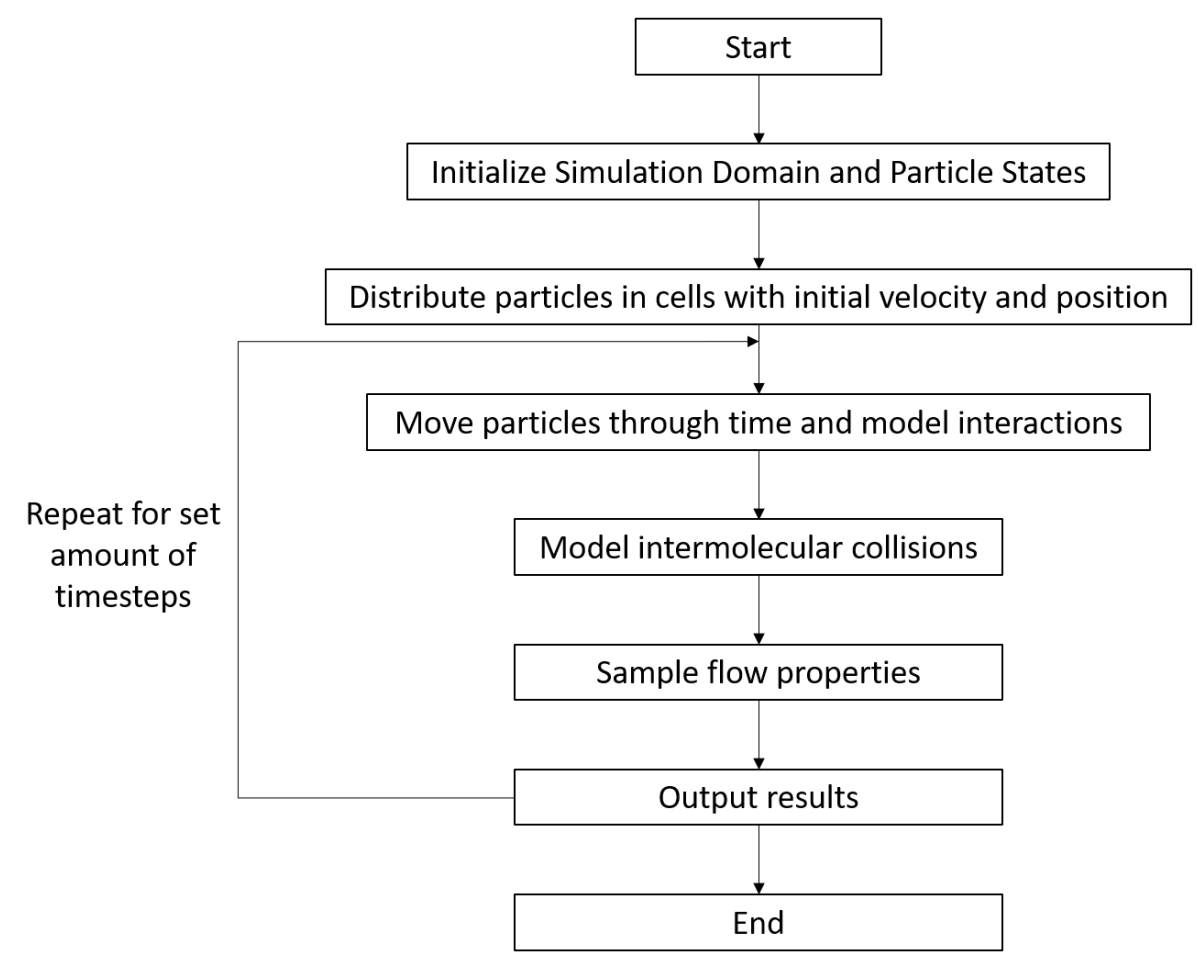

Figure 2.7: DSMC Simulation Process

on to existing features within the code with the efficiency of $\mathrm{C}++$. While it is designed to be able to be used in either traditional desktop computers or parallel computing environments, the handling of parallel communication is generally more efficient. The modular design of the package as well as ease of use makes SPARTA [15] a popular open-source choice that is akin to NASA's internal DSMC software, DAC [37]; however, even with the high-efficiency of SPARTA, the biggest drawback with DSMC remains the computational demand needed to produce high fidelity results, as completing a large amount of simulations needed for probablistic re-entry characterizations in a practical timeframe near impossible [15]. 


\section{Chapter 3}

\section{Methodology}

For this project, different sets of neural networks are created, each for the following case studies:

- Determining the ability of a neural network to learn and reproduce a closed form heating distribution on a sphere.

- Implementing DSMC data into a neural network to reproduce heating distributions on shape primitives in flow with no attitude variations in the $2-4 \mathrm{~km} / \mathrm{s}$ velocity range.

- Repeating the above study for the $6-8 \mathrm{~km} / \mathrm{s}$ velocity range.

- Demonstrate the feasibility of modeling heating distributions on shapes of different sizes.

- Demonstrate the feasibility of modeling heating distributions on a cube with varying orientations (subject of future work).

\subsection{Geometries and 3D Meshing}

The studies for head-on flow focsues on three shape primitives: a sphere, a cube, and a cylinder. The cylinder has two different flow cases: one with the flow facing the circular face and the other with the flow facing the rounded face, shown in Figure 3.1. Since the Knudsen 
Number is a ratio of the distance between molecules in the flow and the characteristic length of the object, the characteristic length of each shape except the cube is one for the sake of simplicity. For this study, the cube has a characteristic length of two in order to validate the scaling model for a side length of one. The Knudsen Number for the cube was then scaled appropriately to make direct comparison to each shape appropriate.

Part of ensuring that accurate flow phenomena is captured for each geometry in DSMC is ensuring that the 3D object created for the simulation is properly meshed. For all of computational fluid dynamics, proper object meshing is imperative to capture important features of interest during the simulation. As such, unstructured meshing, where mesh elements are not indexed and placed systematically, is generally better for CFD/DSMC simulations [42]. With unstructured meshes, the elements are usually triangular as compared to quadrilaterals for structured meshes since the detail of element placing is usually more flexible with triangular elements.

For structured meshes, problems can occur at the vertices of each element causing skewed or inaccurate results. For unstructured meshes, greater control of mesh sizing can be employed allowing for a better mesh defined by the user; however, there is a trade-off that must be reached: The mesh resolution must be high enough that the geometry and any important features are accurately captured, but must not be so detailed that it overcomplicates the deep neural network. Since each mesh element is an independent output of the neural network, increasing the amount of mesh elements would exponentially increase the amount connections between nodes which would eventually make the model intractable. Furthermore, it is important to note that once an object mesh is created, the mesh cannot change as this would require the deep learning model to be retrained. To extend the concept to shape primitives of different sizes, a mesh scaling technique can be adopted as demonstrated in the results section. 


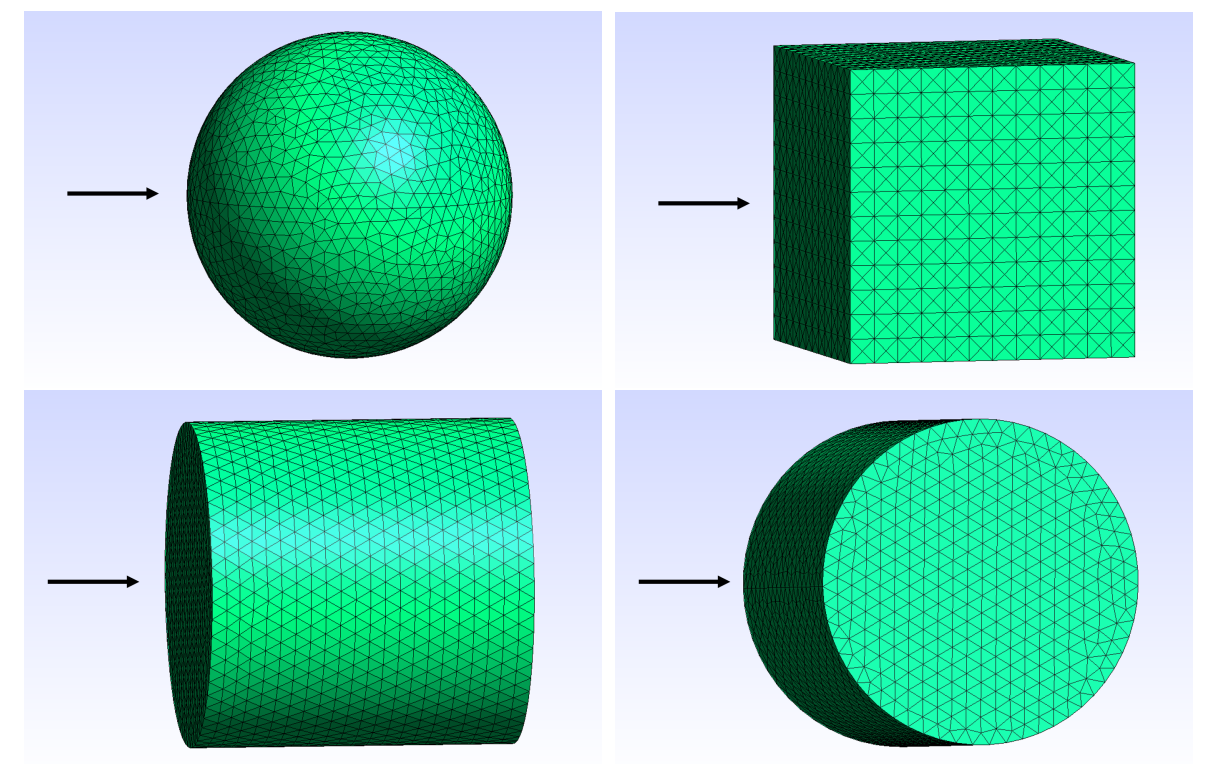

Figure 3.1: Flow Directions for Shape Primitives

In this project, Gmsh, an open-source, finite-element mesh/solver is used [46]. Gmsh allows for different types of meshing algorithms under structured, unstructured, and hybrid meshes to best suit the needs of the developer and the geometry. As mentioned above, unstructured meshes are preferable for CFD/DSMC simulations and Gmsh provides modules that allow for versatile creation of unstructured meshes including a module that allows a developer to manually place mesh points for their geometry. For all shapes in the study, a Blossom-Quad algorithm is used [47]. Table 3.1 shows the amount of mesh elements (corresponding directly to the size of the output layer of the Neural Network) generated by Gmsh.

\subsection{Simulation Database}

The ultimate goal of deep learning is to produce a model that given a set of inputs, is able to generate an output with the same level of accuracy as the data it was trained on. To this end, 
Table 3.1: Shape Primitive Composition (\%)

\begin{tabular}{lr}
\hline Shape & \# Elements \\
\hline Sphere & 3196 \\
Cube & 3456 \\
Cylinder & 3954 \\
\hline
\end{tabular}

the data provided to the deep learning model for training must be wholly representative of the phenomena at hand. In this case of atmospheric re-entry, this data must reflect a diverse set of atmospheric flow conditions corresponding to their respective heating distributions. More specifically, the dataset should completely span both the transition regime and covers typical re-entry speeds and freestream conditions seen in the altitudes corresponding to transition flow. For this study, the three flow conditions chosen to characterize the flow were the Knudsen Number, freestream temperature, and freestream velocity. First, a neural network was developed to test the ability of deep learning to accurately learn and reproduce a closed form solution for a sphere in the continuum regime. The stagnation point equation used is Eq. 2.15 and the function used to map the distribution is Eq. 2.17 The range of input values to be used in the neural network is:

$$
\begin{gathered}
K n \in\{0.0005,0.05\} \\
T \in\{500,2000\} \mathrm{K} \\
V \in\{2,8\} \mathrm{km} / \mathrm{s}
\end{gathered}
$$

With these ranges, 75 training and 50 validation flow cases were generated via Latin hypercube sampling which aims to statistically generate a perfectly near-random sample given a number of dimensions and the minimum and maximum values that each dimension can 
take [43]. This sampling methodology is typically used to generate samples for monte-carlo simulations as this has been shown to reduce computational effort converge on a solution [44]. In a two dimensional input space, the sampling algorithm is based on the Latin square design which aims to generate a sample in each row and column of a used defined space. In three dimensions and above, the Latin square becomes a Latin hypercube. Using this sampling methodology to generate flow cases ensures that each case not only has practically identical weighting to one another and that no flow cases are favored in training or that no additional biases are introduced, but also that the entire input space is adequately represented. Figure 3.2 shows the distribution of training and validation input samples as well as an example of a Latin Hypercube Sample.

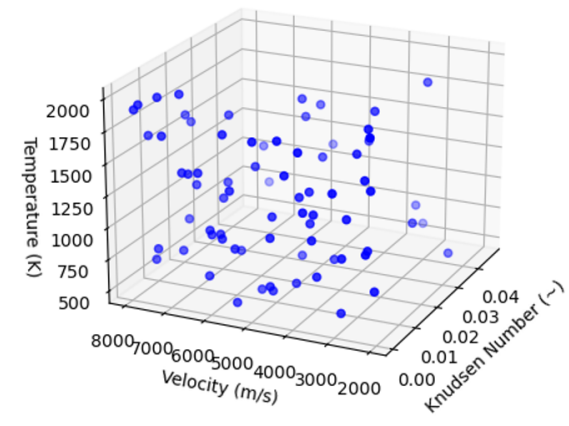

Training

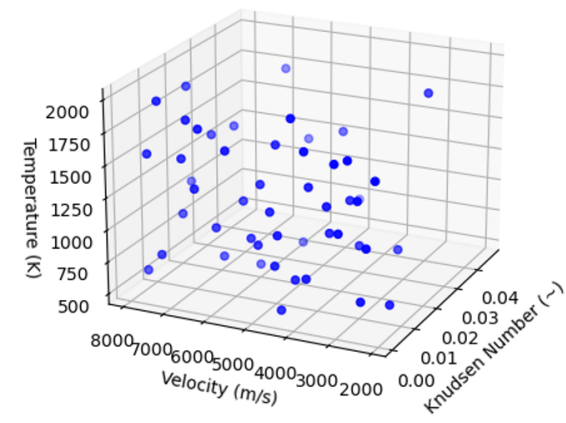

Validation

Figure 3.2: Feasibility Study Input Distribution

Once the feasibility of using neural networks to learn a closed form solution is demonstrated, a similar approach was taken to implement DSMC data into the neural network. To determine suitable values for the Knudsen Number, a range of altitudes that spans the transition regime is determined:

Alt $\in\{85,120\} \mathrm{km}$ 
It is within this altitude range that density and temperature can be extracted from NASA's MSIS-E-90 model to determine the mean free path of the molecules in the atmosphere, and subsequently, the Knudsen Number at a given altitude [45]. The range of Knudsen Number, temperature, and velocity determined for the flow condition dataset are as follows:

$$
\begin{gathered}
K n \in\{0.01,1\} \\
T \in\{200,2000\} \mathrm{K} \\
V \in\{2,4\} \mathrm{km} / \mathrm{s}
\end{gathered}
$$

For each shape primitive, the same randomly generated flow conditions (100 for training and 25 for validation) were used to initialize the DSMC simulations. It is important to note that these ranges were used to generate samples for an initial study as a new sample was generated to include velocity ranges more similar to those seen in atmospheric re-entry (including orbital speeds). As such, the new ranges for that dataset are as follows:

$$
\begin{gathered}
K n \in\{0.01,1\} \\
T \in\{200,2000\} \mathrm{K} \\
V \in\{6,8\} \mathrm{km} / \mathrm{s}
\end{gathered}
$$

Since re-entering objects do not keep a constant orientation, models must be developed to account for the tumbling of a re-entering spacecraft or space debris. In this study, neural networks to model the tumbling motion of a cube are created for demonstration purposes. Due to the attitude dependence of a cube, the dimensions for the training samples increases from three to six, to include the Euler angles of roll, pitch, and yaw. 


$$
\begin{gathered}
\gamma \in\{-180,180\}^{\circ} \\
\beta \in\{-90,90\}^{\circ} \\
\alpha \in\{-180,180\}^{\circ}
\end{gathered}
$$

As was accomplished above, a similar Latin hypercube sample was generated to obtain a random distribution of values for object attitude. Due to the increased complexity of including the object orientation in the models (a three dimensional input space now becomes a six dimensional input space), it is expected that more samples are needed for training in order to adequately learn the input-output relationships. As such, 200 samples were generated for training. Similar to previous datasets, the models are validated on 25 samples.

Finally to account for objects of different sizes, the neural network created for the cube is validated on the cube geometry whose side length is halved. This is accomplished by randomly choosing a validation case, doubling the Knudsen Number, and comparing the evaluation against the DSMC simulation for the smaller cube. The flow case chosen is:

$$
\begin{gathered}
K n=0.32 \\
T=1544.87 \mathrm{~K} \\
V=3.18 \mathrm{~km} / \mathrm{s}
\end{gathered}
$$

\subsection{Data Processing}

To first improve machine learning performance, input and output normalization is imperative for increased learning and faster convergence. For the input data, the data was $\min / \max$ normalized, defined as: 


$$
z=\frac{x-\min (X)}{\max (X)-\min (X)}
$$

For the output data, the values were standard normalized, defined as:

$$
z=\frac{x-\mu}{\sigma}
$$

To further improve performance on the neural networks, each shape had models that corresponded to each of the faces of the 3D geometry. For the cube, a model was trained for each of the six faces. For the cylinder, a model was trained for each of the top and bottom faces as well as one for the curved surface. This is done to reduce and control the output layer size and to reduce the model complexity, and improve accuracy. For the sphere in the new $6-8 \mathrm{~km} / \mathrm{s}$ dataset, the geometry was split into their quadrants.

\subsection{Machine Learning}

\subsubsection{Gaussian Process Regression Modeling}

The aim of GPR is to create a distribution of potential functions that fit the given dataset according to a Gaussian Normal Distribution. The function that has the highest likelihood of fitting the data (maximum likelihood estimation) is then chosen as the representative model [48]. For Python, the scikit-learn library offers a premade Gaussian process regressor with modular functions to include different kernels and noise estimation tools which can automatically output standard deviation bounds, which is useful for uncertainty quantification [49]. Here, the inherently non-parametric approach only requires the definition of a kernel function. For this study, the Matern covariance function (kernel) is used as it generally 
provides a better approximation of nonlinear functions with the appropriate definition of the smoothness parameter, $\nu[50]$. The matern kernel is defined as

$$
k\left(x_{i}, x_{j}\right)=\frac{1}{\Gamma(\nu) 2^{\nu-1}}\left(\frac{\sqrt{2 \nu}}{l} d\left(x_{i}, x_{j}\right)^{\nu}\left(\frac{\sqrt{2 \nu}}{l} d\left(x_{i}, x_{j}\right)\right)\right.
$$

Where $\nu=1.5$, indicating a once-differentiable function.

To estimate the noise for the model, a further White Kernel was added, where the noise is estimated according to an independent and identically normally-distributed function [51]. The White Kernel is defined as:

$$
k\left(x_{i}, x_{j}\right)=\text { noise level if } x_{i}=x_{j} \text { else } 0
$$

The noise level parameter equals the variance of this noise.

\subsubsection{Artifical Neural Networks}

Deep Learning has become a very popular tool for approximating input-output relationships for highly complex systems. Deep Learning primarily makes use of different neural network architectures to learn and predict complex functions. Ultimately, the goal of any supervised learning problem is to train on a set of given inputs and outputs, and formulate a functional relationship that generalizes well enough so that given a new set of inputs, the model is able to generate an accurate prediction. In this study, a fully-connected neural network is used to model a full heating distribution over the primitive surface. 


\section{Hyperparameter Optimization}

A key challenge for deep learning is the identification of the optimal set of network architectures and hyperparameters due to the large amount of controllable parameters in a neural network. Due to the simplicity of the closed-form function used for the initial feasibility study, a simple model was created where the architecture was manually defined; however, in DSMC simulations, statistical noise is introduced due to the stochastic processes used to solve the particle flow and motion, which increases the complexity of the data to be modeled. For this reason, manually creating a suitable architecture is quite difficult. A solution to this is based in NAS and hyperparameters optimization algorithms. In recent years, various NAS libraries (AutoKeras [53], Keras Tuner[55], AutoML[52] ,etc.) have been developed that provide an easy-to-use tool for identifying the optimal set of hyperparameters for a given dataset.

Within NAS, there are three components: the search space, the search algorithm, and the performance estimation strategy [38]. The search space determines which designs can theoretically be represented. Prior knowledge of typical attributes of well-suited architectures can be used to limit the size of the search space and simplify the search. This, however, introduces a human bias which may limit the discovery of unique architectural building blocks that go beyond human expertise. The search strategy explains how to go about exploring the search space (which is often exponentially large or even unbounded). It contains the basic exploration-exploitation trade-off in which it is desirable to locate well-performing designs quickly while avoiding early convergence to a region of inferior structures. The goal of NAS is usually to find designs that can perform well on data that hasn't been seen before. The easiest way is to train and validate the architecture on data, however this is computationally expensive and restricts the number of architectures that may be investigated. As a result, recent research has focused on devising strategies for lowering the cost of these performance 
estimates (performance estimation strategy). Figure 3.3 shows how these components work with each other during the search process.

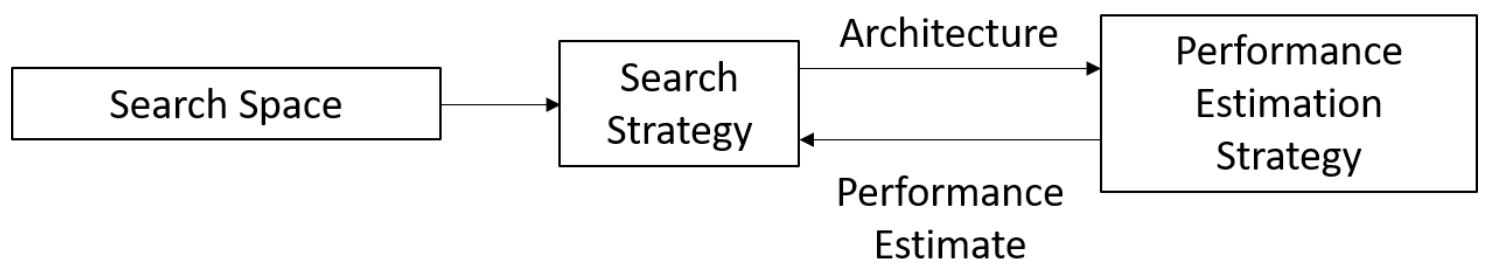

Figure 3.3: Neural Architecture Search Process

There are many search algorithms used for NAS libraries, but two popular ones (also used for this study) are Bayesian Optimization [39] and Hyperband [40]. The Bayesian optimization algorithm is popular compared to others because it takes a more elegant approach to converging on the best deep learning model parameters [39]. Specifically, Bayesian Optimization leverages results from previous search trials to determine better parameters (prior information) for future trials. First, an evaluation period is initialized where hyperparameters are randomly sampled for a specified amount of training trials. After the initial trial, the algorithm converges on the best hyperparameters based on a Bayesian algorithm. This generally will make more efficient use of time as other methods (random or grid search) are more "brute force" methods that do not account for past trials; making convergence in a practical amount of trials less likely. Conversely, this algorithm is sometimes prone to exploding gradients which may cause the algorithm to crash and not function properly. Such an issue is still an active bug in Keras Tuner that is undergoing investigation [41]. In the case that these trials experience the exploding gradients issue, Hyperband is a suitable alternative.

Hyperband is a novel technique that conducts the search in a championship bracket style where potentially suitable models compete against one another until the best model remains [40]. First, a large number of models with randomly-sampled hyperparameters are 
created and trained on a small amount of epochs for the "first round". Then, for subsequent rounds, a user-defined percentage of the best performing models are collected and trained for more epochs. The process repeats until a lone model remains as the best performing architecture. Hyperband generally excels at providing models that perform similarly to the Bayesian optimization algorithm that converge much quicker; however, due to the inherent process in which the Hyperband optimizer tunes the hyperparameters, architectures that may require a large amount of epochs to converge to an optimal solution may not be found since initial models are trained for such a small time. Furthermore, the effectiveness of this algorithm can be increased by expanding the amount of maximum epochs to train the model, as well as amount of trial iterations; however, this generally corresponds to a scaling of computational resources that may become impractical. 


\section{Chapter 4}

\section{Results}

\subsection{Initial Feasibility Study}

Shown in Figure 4.1 are the training and validation results for the initial case study where the neural network is trained to reproduce the Sutton-Graves heat flux equation found in Eq. 2.15. The models show extremely good agreement with the analytical data, boasting an $R^{2}$ that is extremely close to and rounds to 1 (nearly all of the variance in the data captured by the model). A high $R^{2}$ value is to be expected due to the fact that the analytical formulation is noise-free and that there is no expected noise when creating the training and validation data.

To better quantify this performance, Figure 4.2 shows the mean percentage error for each sample in both the validation and training datasets. The errors shown are extremely low, as the model can evaluate both training and validation inputs within $0.6 \%$ error for the entire distribution for values over $Q=100 \frac{w}{m^{2}}$. This threshold is set because minute differences in absolute error may significantly increase the percentage error in a misleading way. The values under this threshold are so low that they are nowhere near critical heating values for re-entering space debris.

Furthermore, the heating distributions are shown Figure 4.3 and 4.4 to demonstrate the ability of the neural network to accurately map the full heating distribution on the sphere. 
The deep learning model is able to capture the stagnation point heating on the center as well as the symmetrical and uniform distribution of heating on the entire surface.
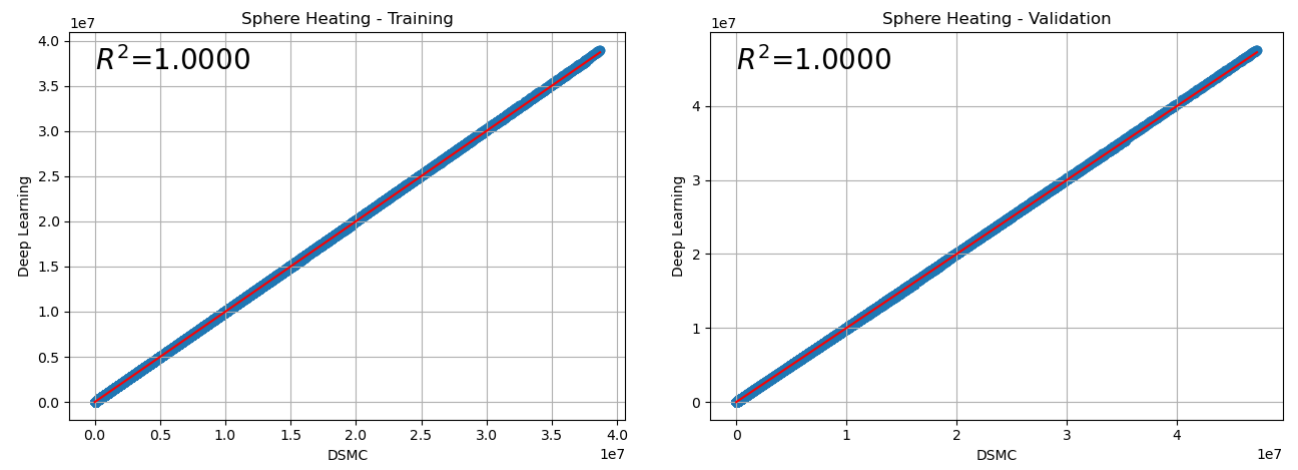

Figure 4.1: Sphere Heating Distribution
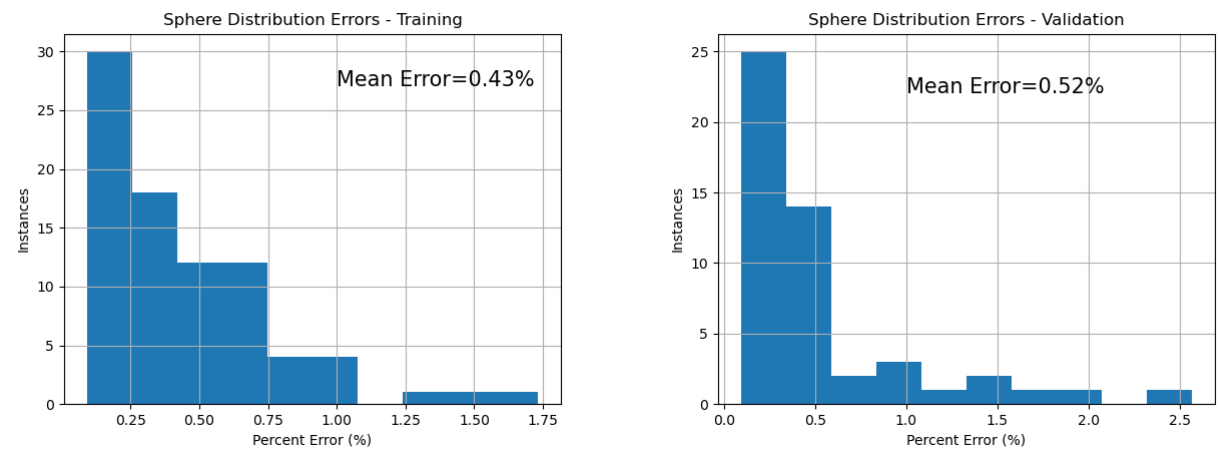

Figure 4.2: Sphere Error

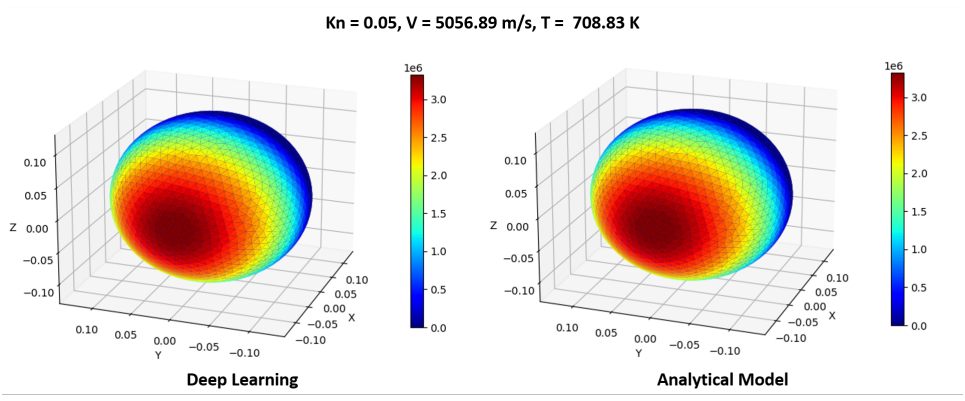

Figure 4.3: Sphere Low Heating Case 


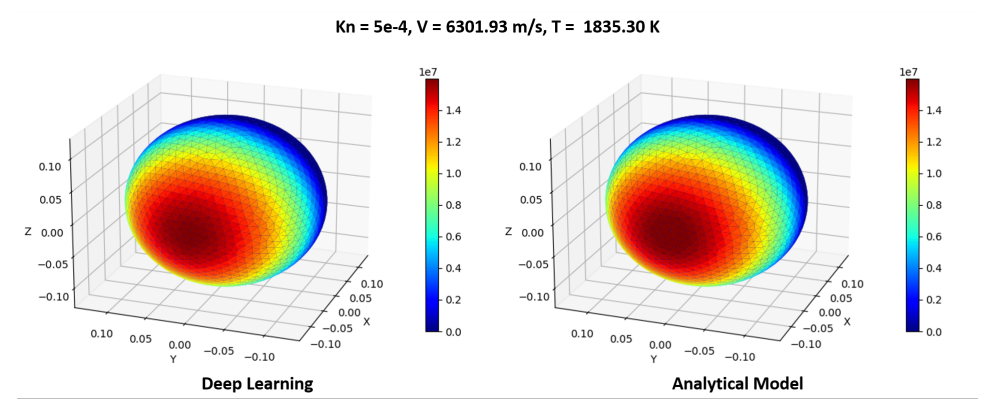

Figure 4.4: Sphere High Heating Case

\subsection{Drag Coefficient - 2-4km/s Velocity Range}

The results of the Drag Coefficient predictions from the Gaussian Process Regression Model is shown in Figure 4.5. As expected, the training data performs extremely well with very low uncertainty bounds; however, much of the same can be said for the validation data as well. Here, a good replication of DSMC data is seen for all shapes as the mean error for each primitive is under $1 \%$. The uncertainty bounds are larger compared to the training data due to the location of the validation inputs within the input space compared to the training inputs. Cases where predictions have higher uncertainty bounds generally correspond to input values that lie close to one of the training inputs, whereas predictions with low uncertainties indicate that the inputs for that specific case lie very close or directly coincide with one of the training inputs. Nonetheless, Gaussian Process Regression Modeling offers a good prediction tool for drag coefficients in re-entry flow. A table summarizing the performance of the GPR is shown in Table 4.1. 
Table 4.1: Drag Coefficient Errors (\%)

\begin{tabular}{lrc}
\hline Shape & Training & Validation \\
\hline Sphere & $0.001 \%$ & $0.75 \%$ \\
Cube & $0.002 \%$ & $0.19 \%$ \\
Cyl. Side Face & $0.002 \%$ & $0.26 \%$ \\
Cyl. Ram Face & $0.002 \%$ & $0.39 \%$ \\
\hline
\end{tabular}
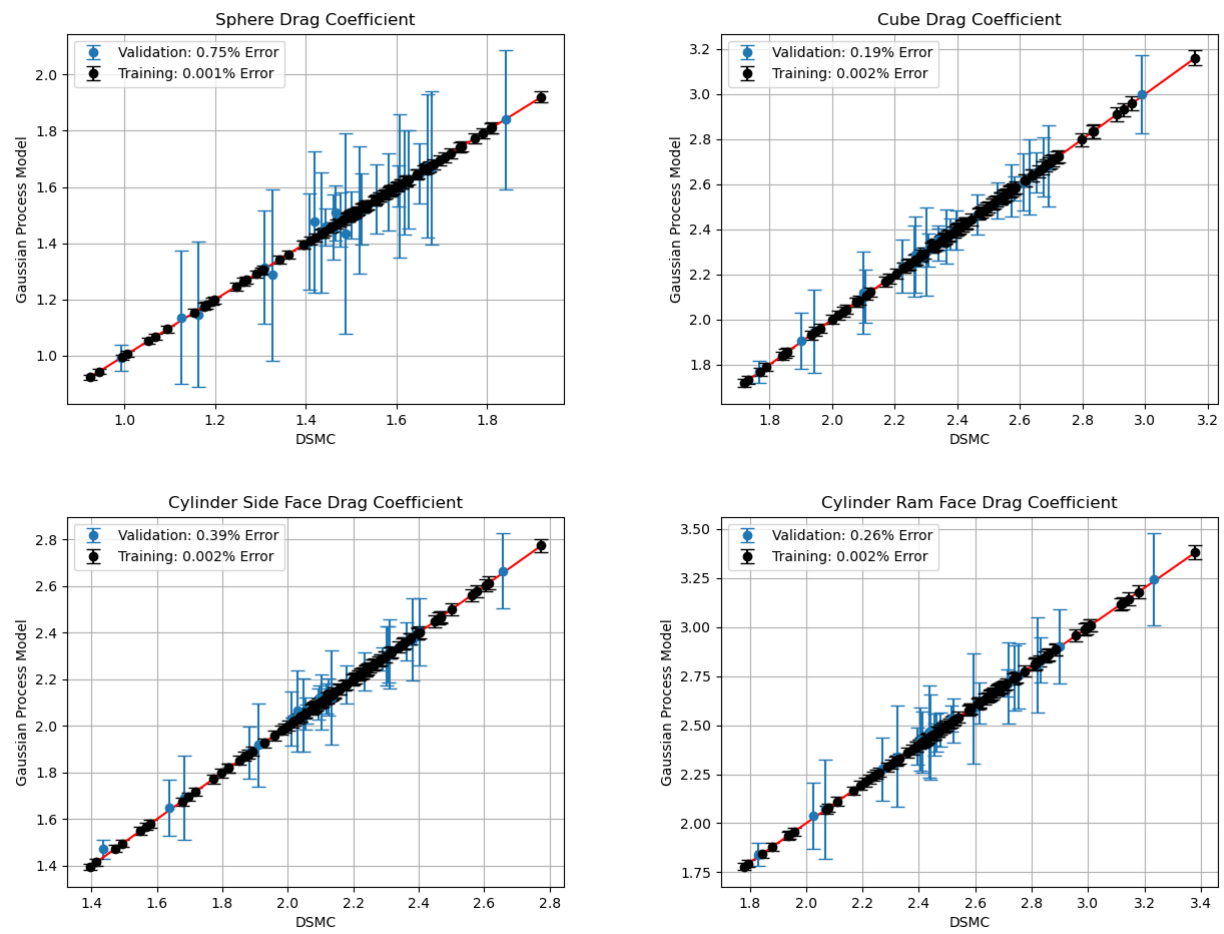

Figure 4.5: Drag Coefficient Results for Each Shape 


\subsection{Drag Coefficient - 6-8km/s Velocity Range}

The Gaussian Process Model performance on the new dataset is summarized in Table 4.2 and shown in Figure 4.6. Similarly to the lower velocity range study, the models perform extremely well on the training dataset and with low uncertainties. On the validation dataset, the performance is similar to that seen in the previous section, with all models predicting within $0.5 \%$ error with similar uncertainty bounds.

Table 4.2: Drag Coefficient Errors (\%)

\begin{tabular}{lrc}
\hline Shape & Training & Validation \\
\hline Sphere & $0.002 \%$ & $0.22 \%$ \\
Cube & $0.008 \%$ & $0.26 \%$ \\
Cyl. Side Face & $0.003 \%$ & $0.40 \%$ \\
Cyl. Ram Face & $0.23 \%$ & $0.009 \%$ \\
\hline
\end{tabular}

\subsection{Heating Distributions - 2-4km/s Velocity Range}

For the full heating distributions, much of the same can be seen as compared to the drag coefficient. For all shapes, the deep learning models offer a good replication of DSMC data in both training and validation data, shown in Figures 4.7, 4.13, 4.17, and 4.21. In each figure, the models trained for each face are combined into the same figure to show the overall performance. For the sphere and the cube specifically, there is very little over- or underfitting to speak of, as most of the deep learning predictions coinciding nearly perfectly with the DSMC data. With the cylinder cases, the side face validation indicates there is some overprediction at higher heating values due to a bias to lower Knudsen Number cases; however, the training data shows a good replication of DSMC results. For the ram face, 

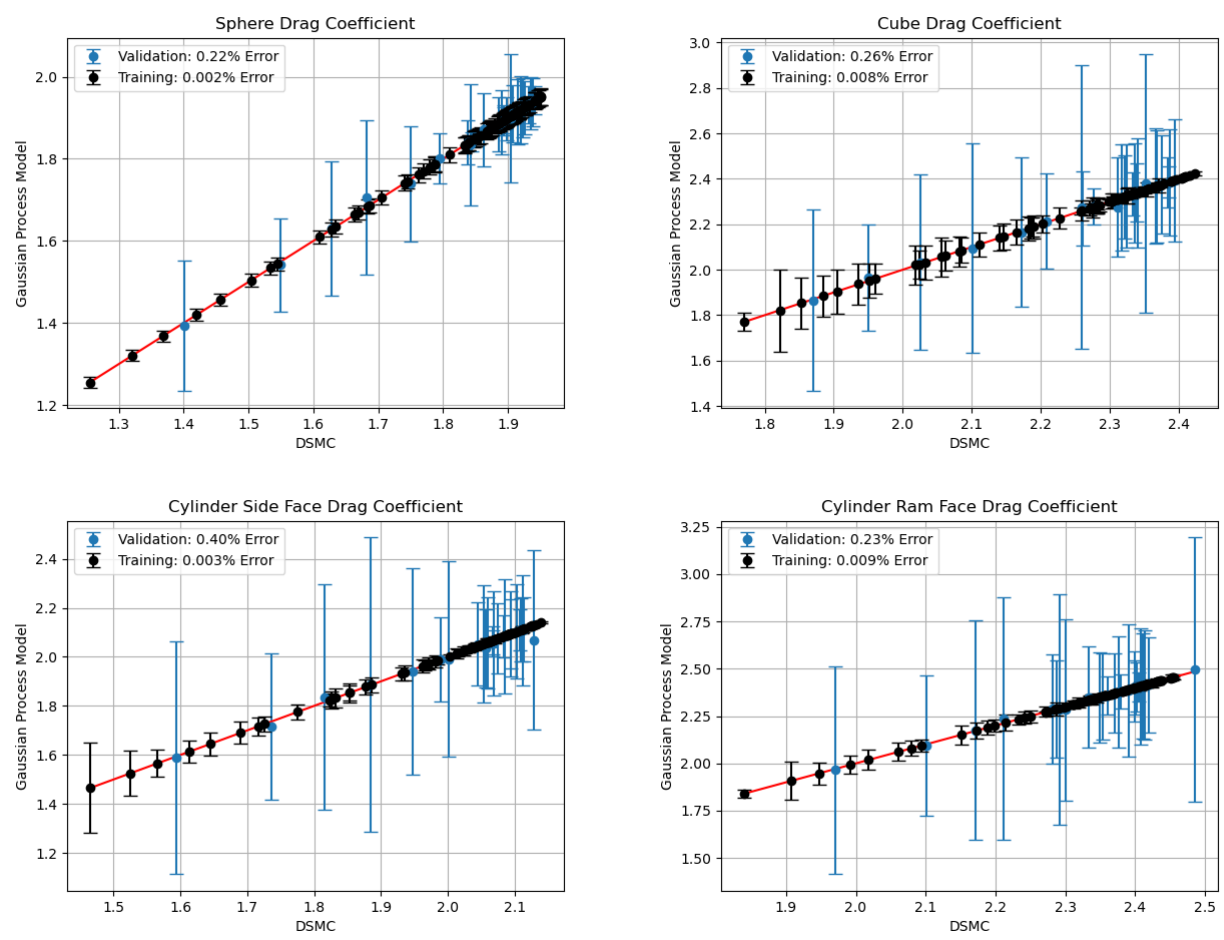

Figure 4.6: Drag Coefficient Results for Each Shape

there seems to be a less tight fit around the 45 degree line than in previous cases; however, the model still performs well nonetheless with all $R^{2}$ exceeding 0.995 (indicating that over $99.5 \%$ of variance is captured in the model).

To quantify the error associated with each distribution, the mean percentage error is shown in Figures 4.8, 4.14, 4.18, and 4.22. For each shape, the mean error for each distribution does not exceed $3 \%$. This is higher than the initial feasibility study due to the introduction of statistical noise in the DSMC simulations. Regardless, the models still perform well and are able to make reasonable predictions for each flow case. The errors and associated $R^{2}$ scores are shown below in Tables 4.3 and 4.4

The visualizations for the low and high heating cases predicted by deep learning as well as the difference between the visualizations is shown in Figures 4.9, 4.11, 4.15, 4.16, $4.19,4.20,4.23$, and 4.24. For the sphere, a comparison is shown between the deep learning 
Table 4.3: Neural Network Training Performance - 2-4km/s Velocity Range

\begin{tabular}{lrc}
\hline Shape & \% Error & $R^{2}$ \\
\hline Sphere & $2.92 \%$ & 0.9987 \\
Cube & $1.06 \%$ & 0.9997 \\
Cyl. Side Face & $1.77 \%$ & 0.9985 \\
Cyl. Ram Face & $1.47 \%$ & 0.9996 \\
\hline
\end{tabular}

Table 4.4: Neural Network Validation Performance - 2-4km/s Velocity Range

\begin{tabular}{lrc}
\hline Shape & \% Error & $R^{2}$ \\
\hline Sphere & $2.94 \%$ & 0.9987 \\
Cube & $1.58 \%$ & 0.9996 \\
Cyl. Side Face & $2.29 \%$ & 0.9954 \\
Cyl. Ram Face & $1.73 \%$ & 0.9996 \\
\hline
\end{tabular}

model and the transition regime bridging function is shown in Figures 4.10 and 4.12. The transition regime model used is found in Eq. 2.16 with the free-molecular and continuum regime contributions coming from Eq. 2.9 and 2.14, respectively. Here, it can be seen that analytical solutions for the whole distribution is modeled as a function of the stagnation point heat flux as shown in previous sections. Furthermore, it is assumed that regardless of flow regime, the distribution would be identically mapped across the entire object; however, this is seen to not be true. It is evident that for higher Knudsen Number flows, the highest heating is much more distributed over the face exposed to the flow. For the high heating case, the heating is much more concentrated in the center, which indicates that as Knudsen Number decreases, the stagnation point heat flux increases exponentially compared to the remainder of the distribution. For the cube and cylinder, the most important feature to be captured that has largely been impossible to model as of date is the areas of higher heating 
around edges and corners due to flow expansion around these edges. For these three shapes, the deep learning model is able to capture this phenomena quite well. Similarly to the sphere, the model is able to predict more evenly distributed values of high heating at the higher Knudsen Number cases and more concentrated points of heat flux at lower Knudsen Numbers. In terms of the difference between the numerical and deep learning models, the difference is largely caused by stochastic noise in the models. Predictably, higher absolute differences are seen in areas of higher heating; but the percentage error is still small.
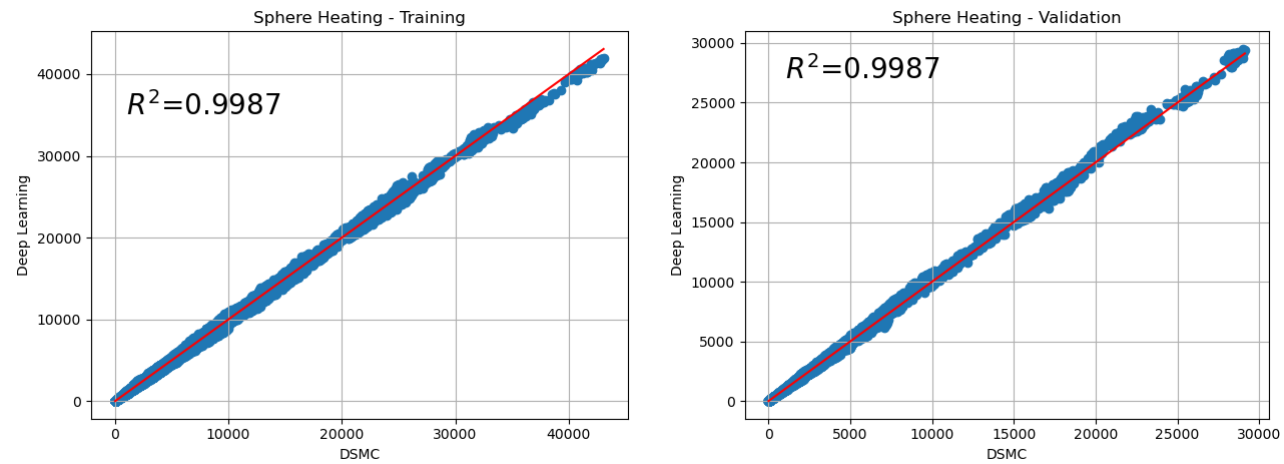

Figure 4.7: Sphere Heating Distribution
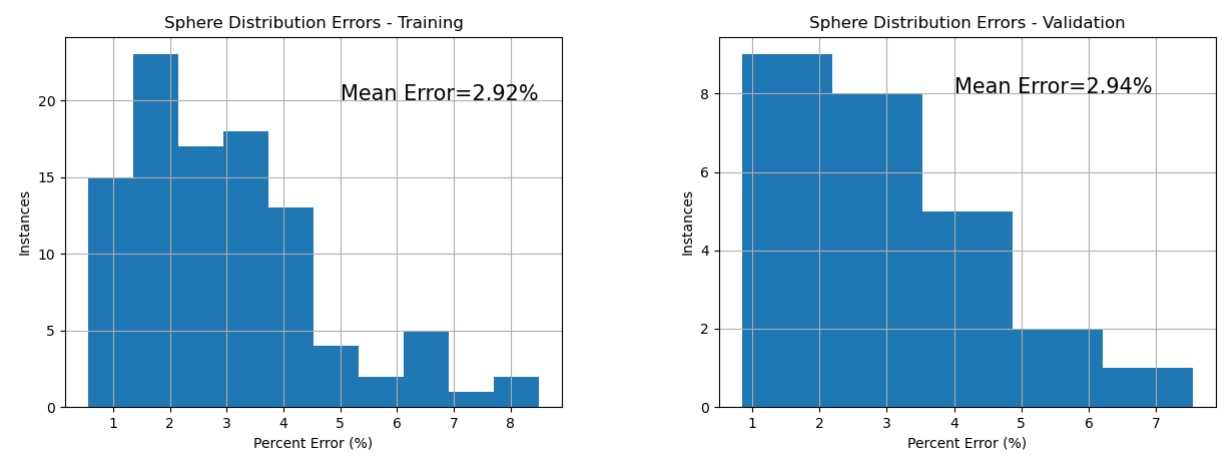

Figure 4.8: Sphere Error 

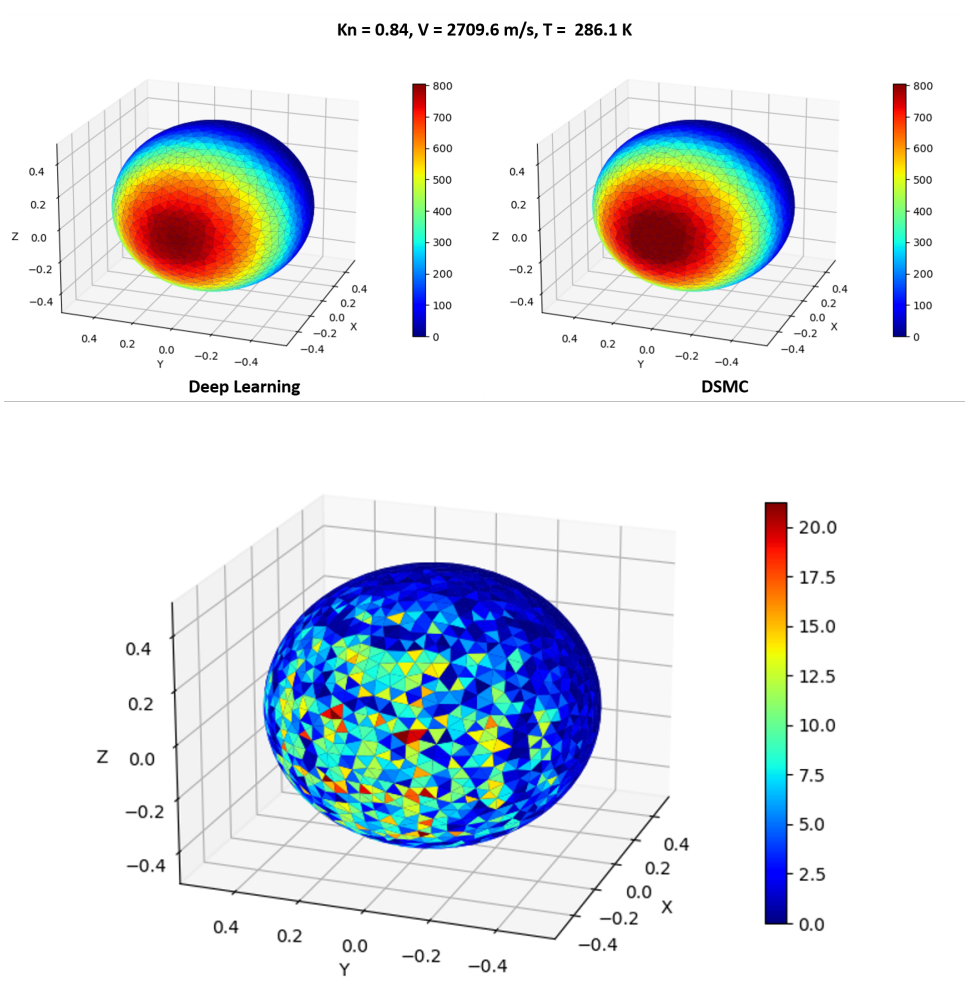

Difference

Figure 4.9: Sphere Low Heating Case

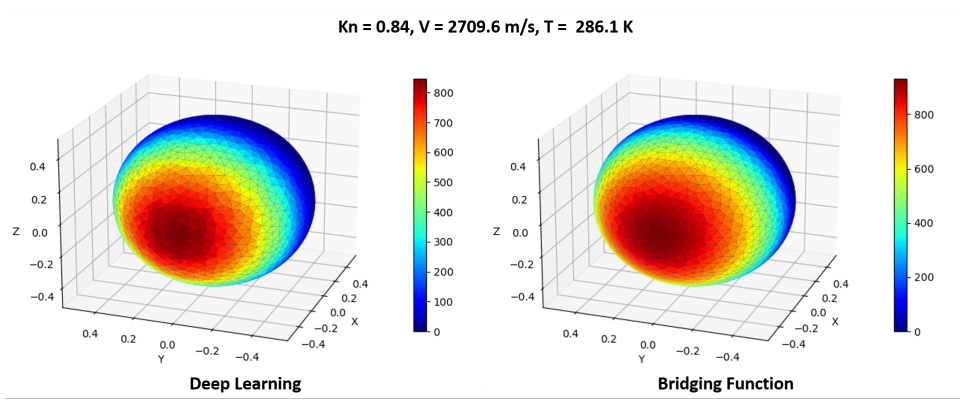

Figure 4.10: Sphere Bridging Function Comparison - Low Heating Case 

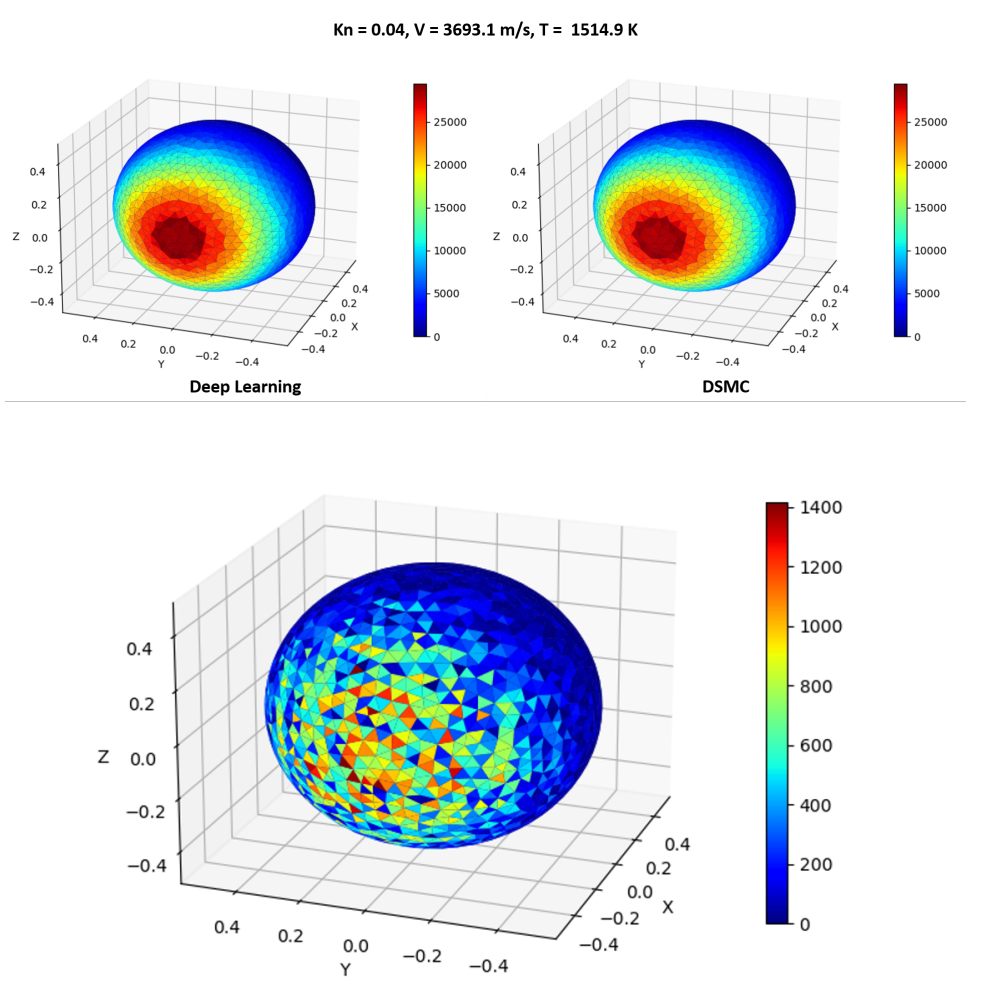

Difference

Figure 4.11: Sphere High Heating Case

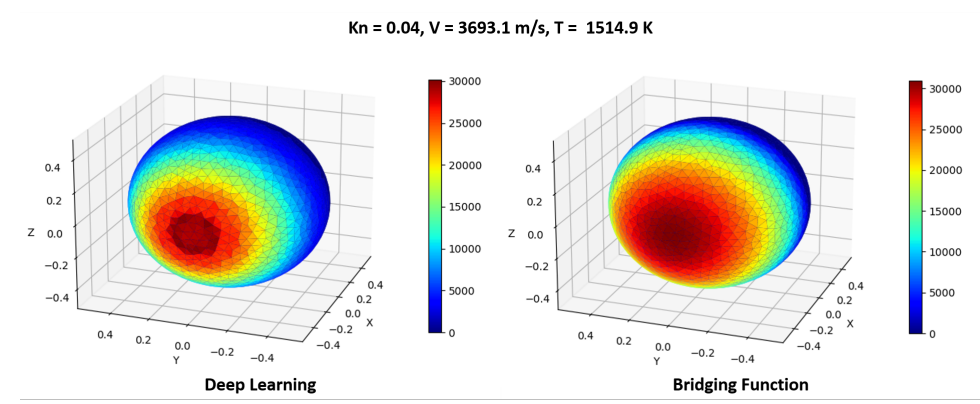

Figure 4.12: Sphere Bridging Function Comparison - High Heating Case 

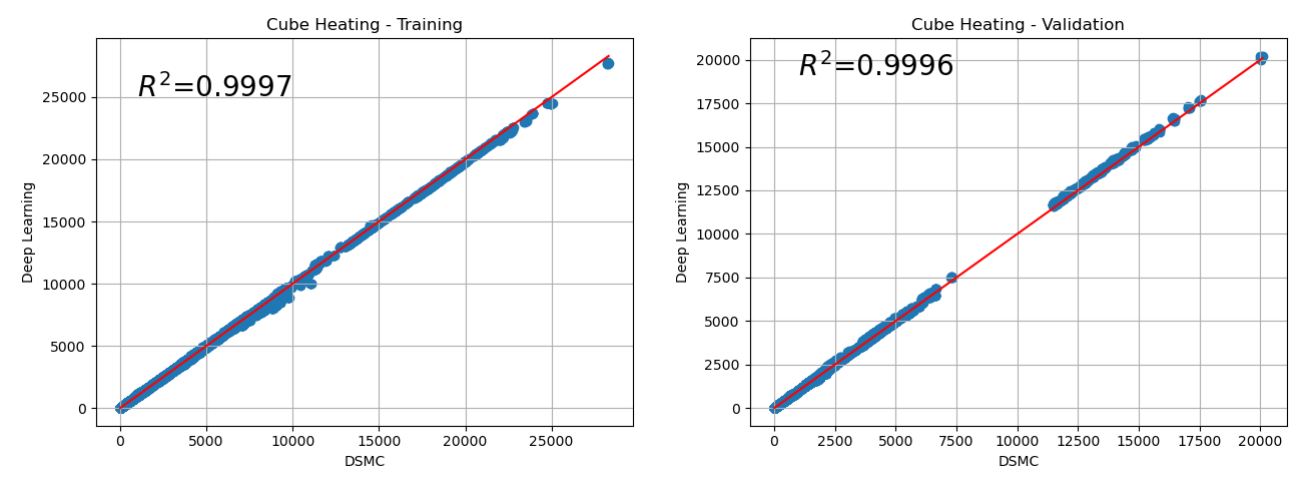

Figure 4.13: Cube Heating Distribution
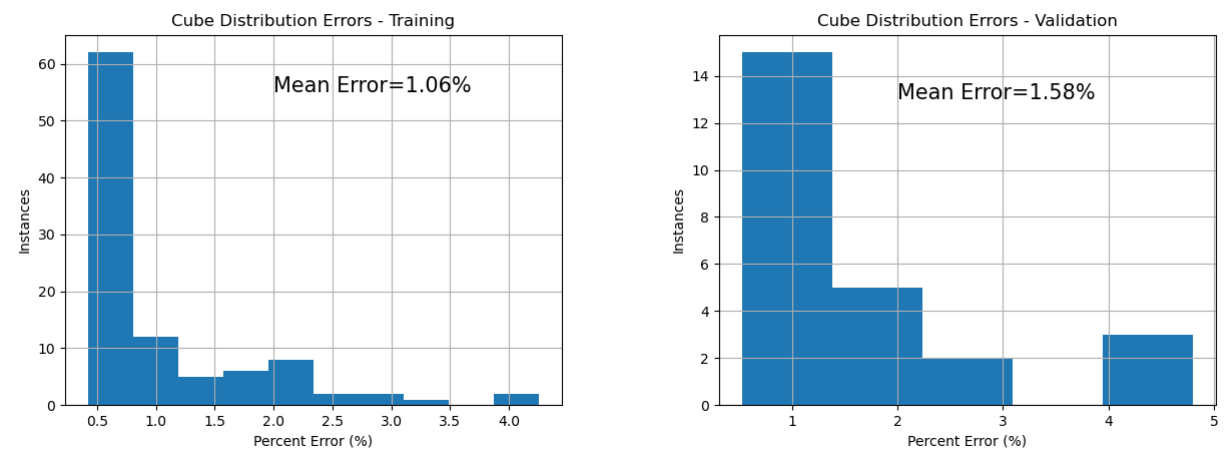

Figure 4.14: Cube Error 

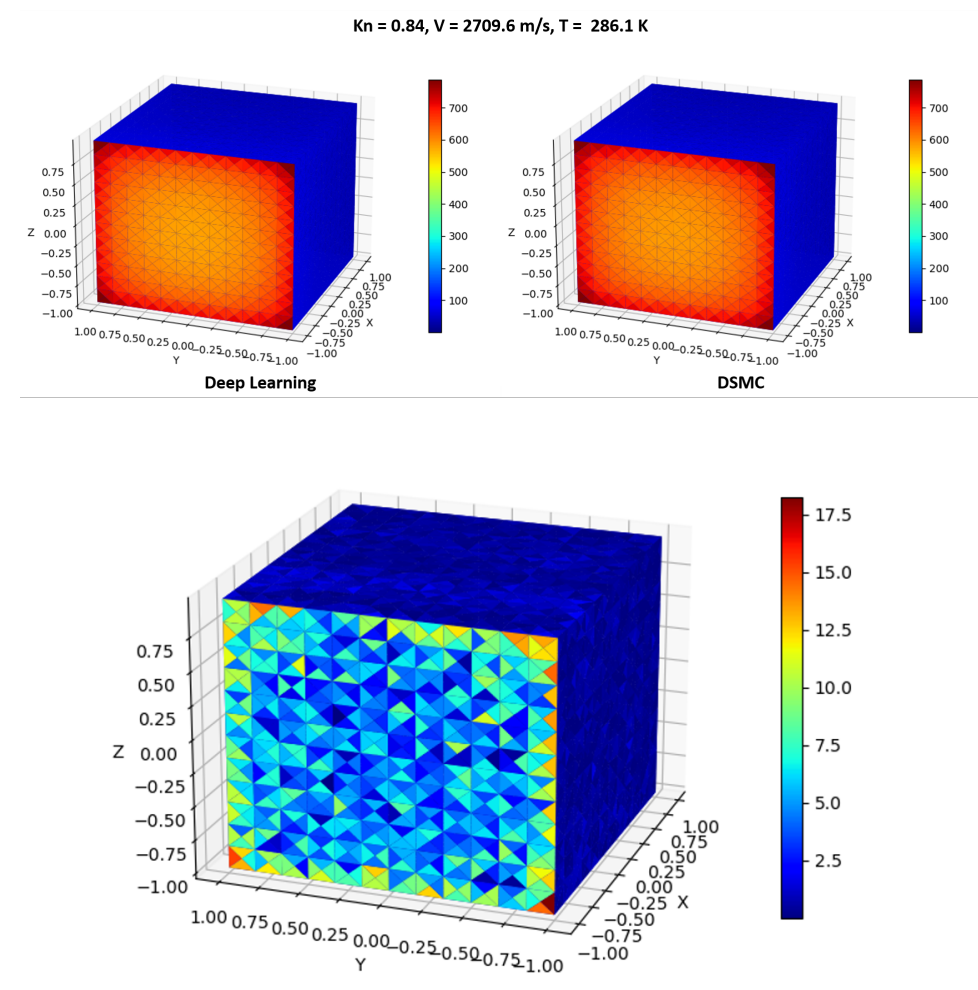

Difference

Figure 4.15: Cube Low Heating Case 

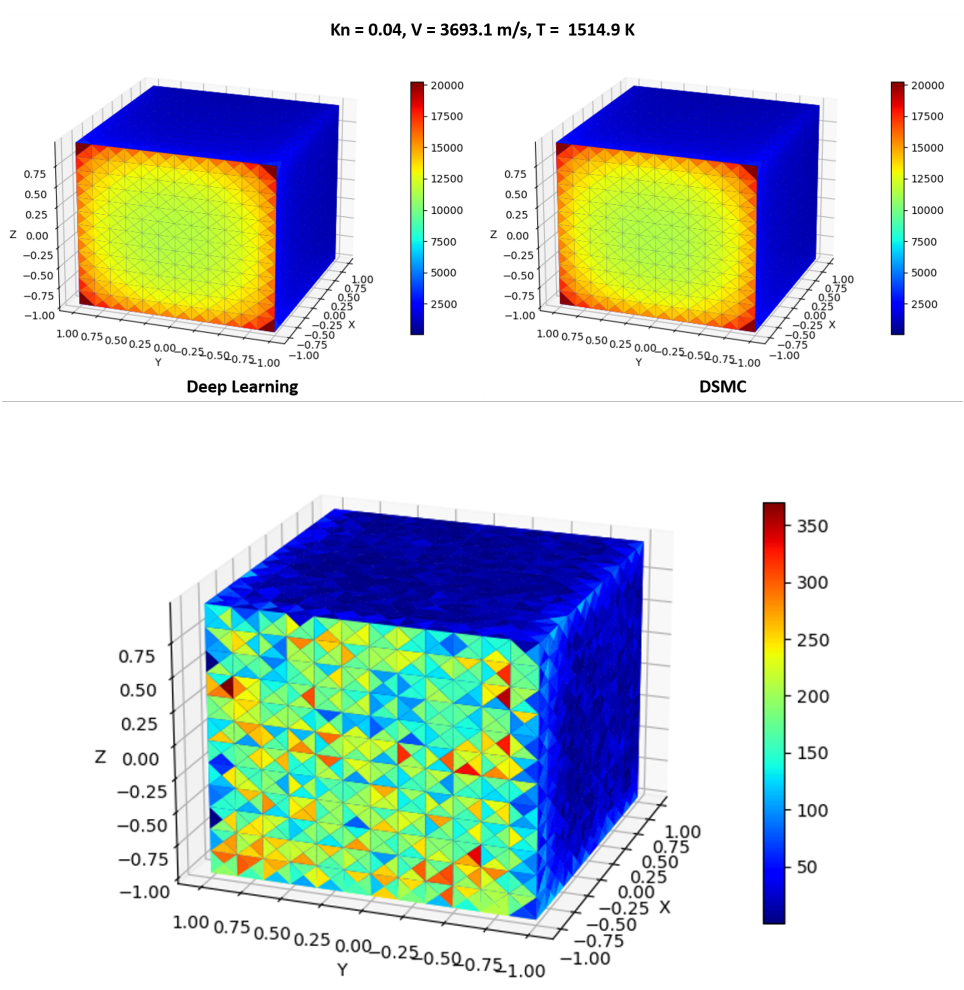

Difference

Figure 4.16: Cube High Heating Case
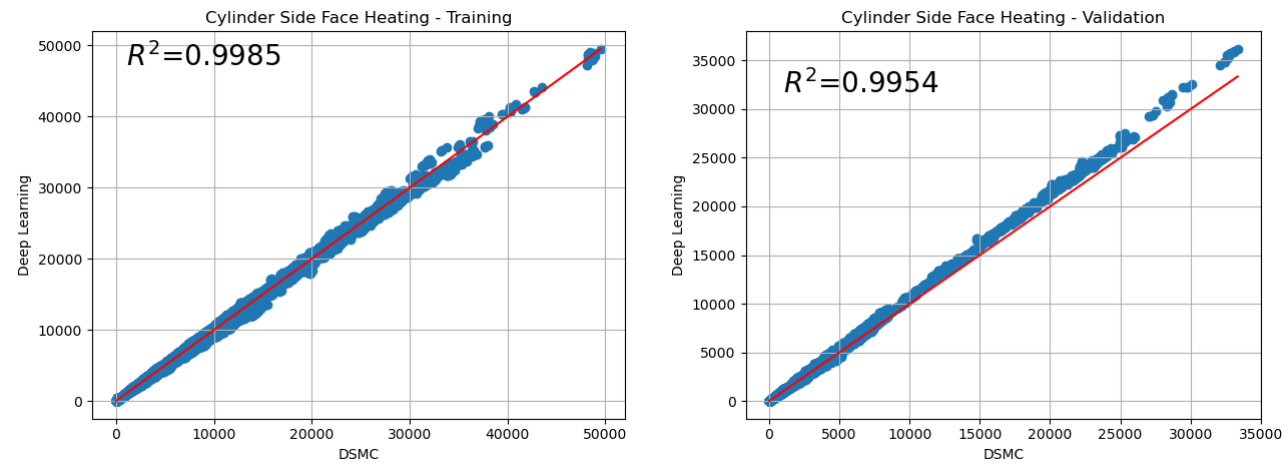

Figure 4.17: Cylinder Side Face Heating Distribution 

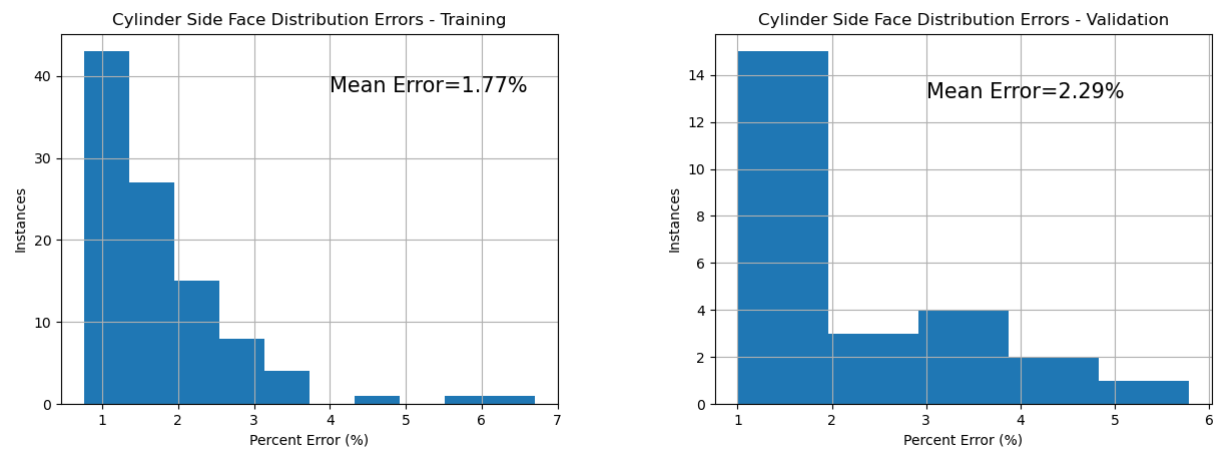

Figure 4.18: Cylinder Side Face Error
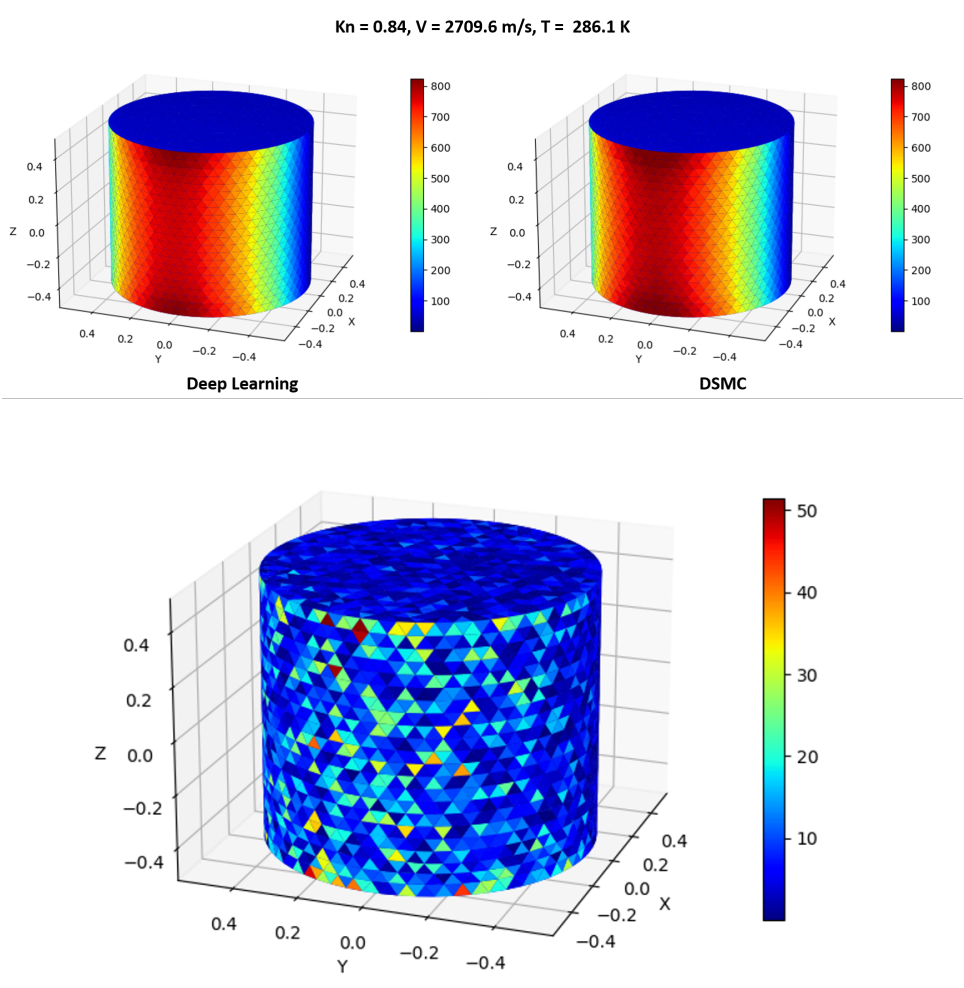

Difference

Figure 4.19: Cylinder Side Face Low Heating Case 

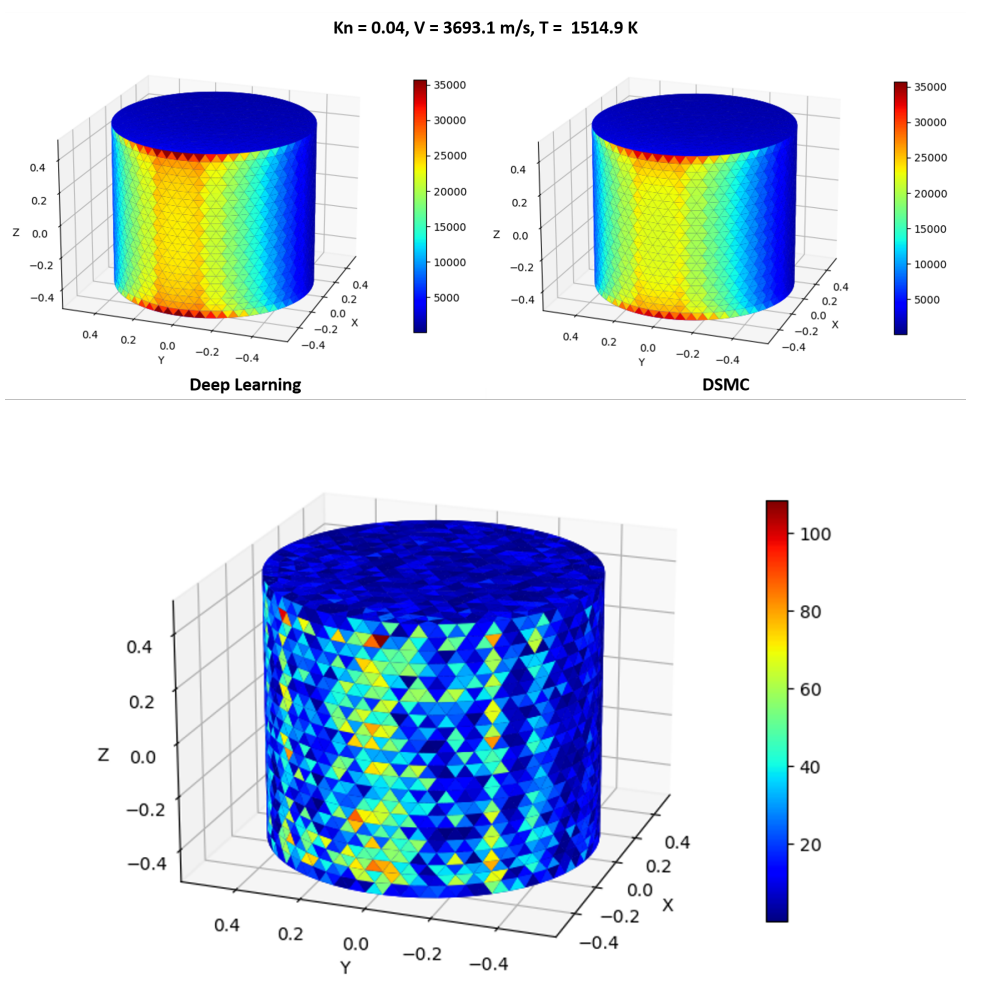

Difference

Figure 4.20: Cylinder Side Face High Heating Case
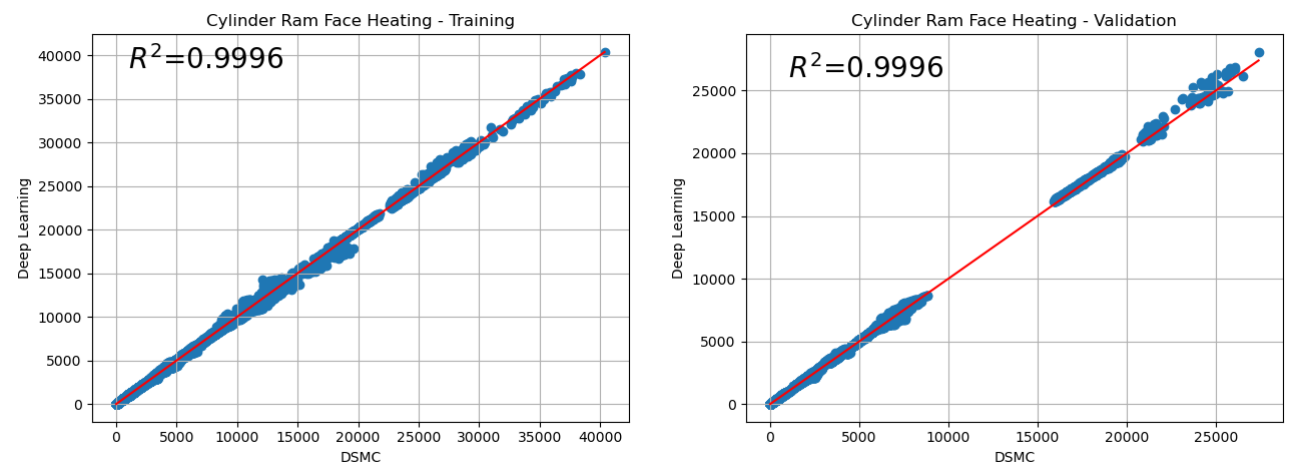

Figure 4.21: Cylinder Ram Face Heating Distribution 

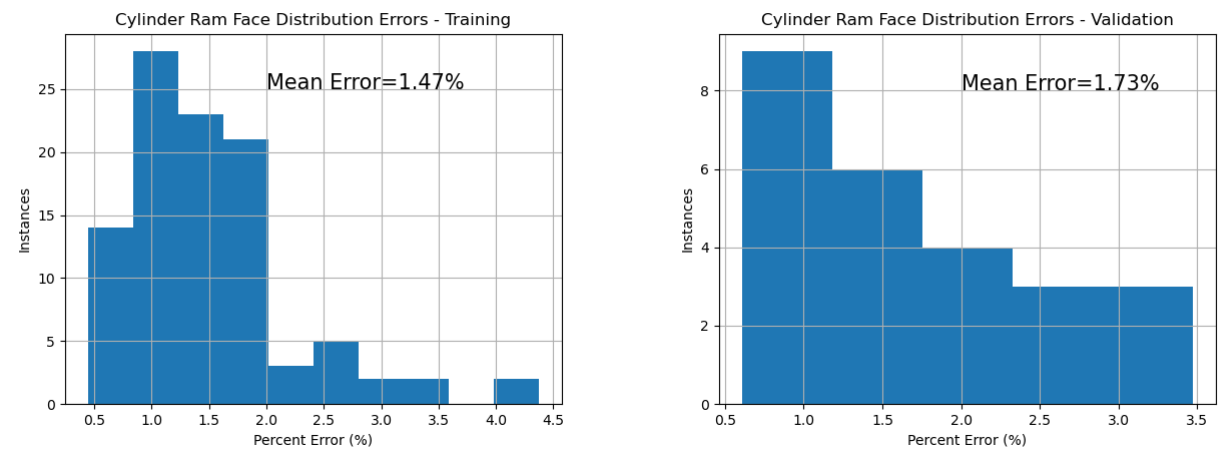

Figure 4.22: Cylinder Ram Face Error
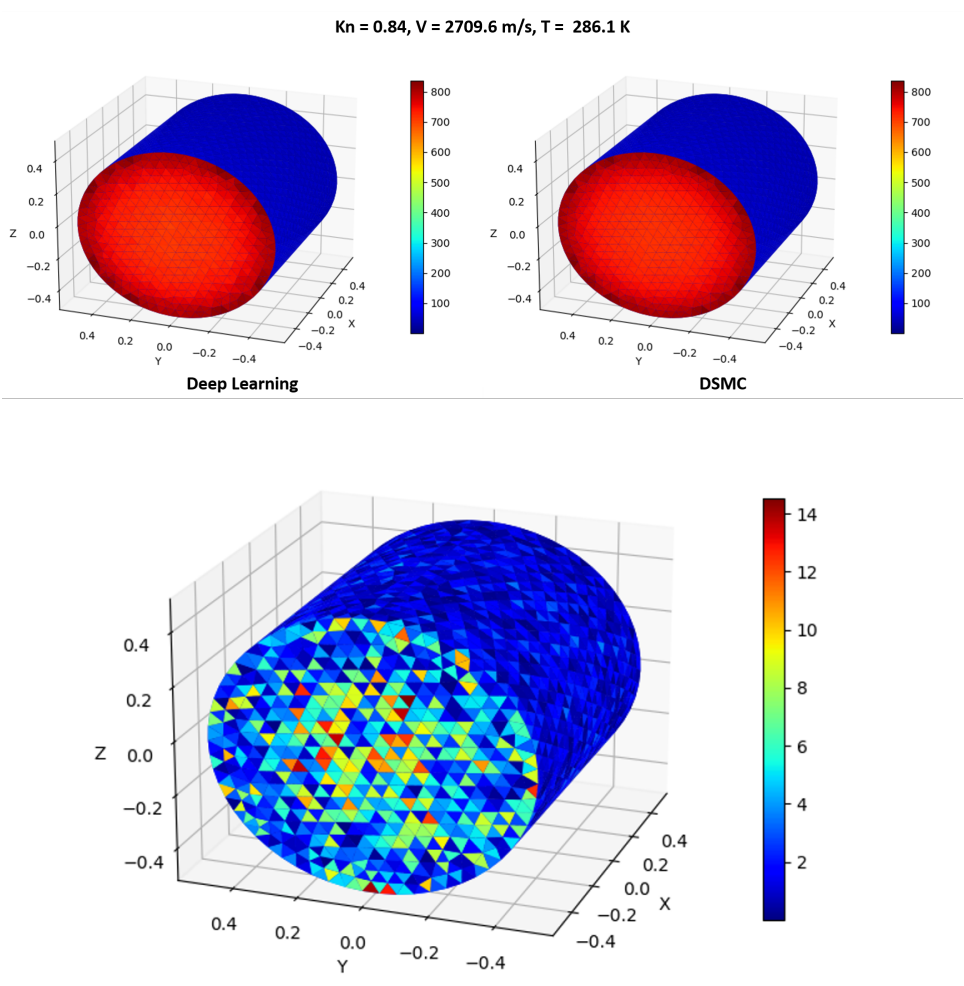

Difference

Figure 4.23: Cylinder Ram Face Low Heating Case 

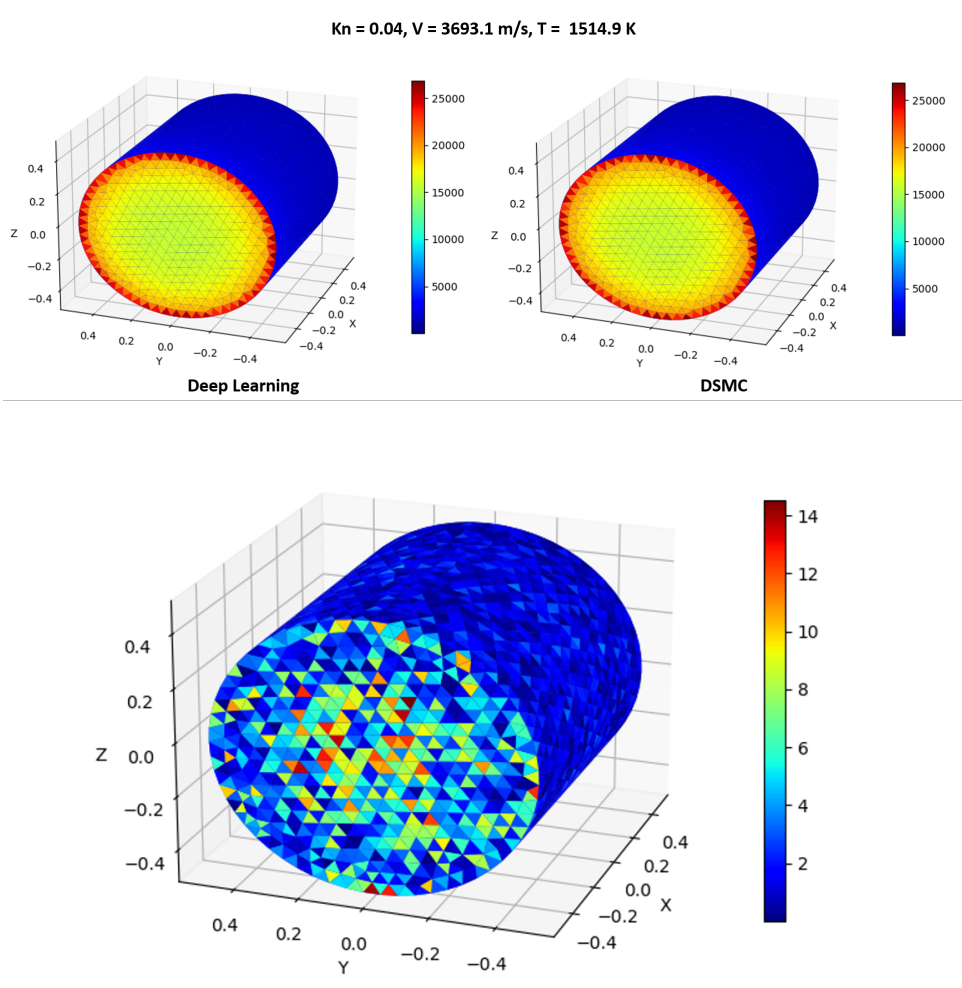

Difference

Figure 4.24: Cylinder Ram Face High Heating Case 


\subsection{Heating Distributions - 6-8km/s Velocity Range}

Similar performance can be seen with the datasets where the velocity ranges are increased to those seen in re-entry and orbital speeds. Like the last section, the $R^{2}$ scores all exceed 0.995 , with the mean errors all falling within 3.5\%. These results are summarized in Tables 4.5 and 4.6. A slightly greater variance can be seen in the predictions, which can be attributed to higher noise seen in the simulations due to the increased velocity of particles modeled. The model performance, along with the associated errors and visualizations can be seen in Figures 4.25 through 4.42

Table 4.5: Neural Network Training Performance - 6-8km/s Velocity Range

\begin{tabular}{lrc}
\hline Shape & \% Error & $R^{2}$ \\
\hline Sphere & $3.22 \%$ & 0.9958 \\
Cube & $1.73 \%$ & 0.9995 \\
Cyl. Side Face & $3.23 \%$ & 0.9977 \\
Cyl. Ram Face & $2.83 \%$ & 0.9983 \\
\hline
\end{tabular}

Table 4.6: Neural Network Validation Performance - 6-8km/s Velocity Range

\begin{tabular}{lrc}
\hline Shape & \% Error & $R^{2}$ \\
\hline Sphere & $3.34 \%$ & 0.9958 \\
Cube & $1.87 \%$ & 0.9986 \\
Cyl. Side Face & $3.38 \%$ & 0.9970 \\
Cyl. Ram Face & $2.83 \%$ & 0.9982 \\
\hline
\end{tabular}



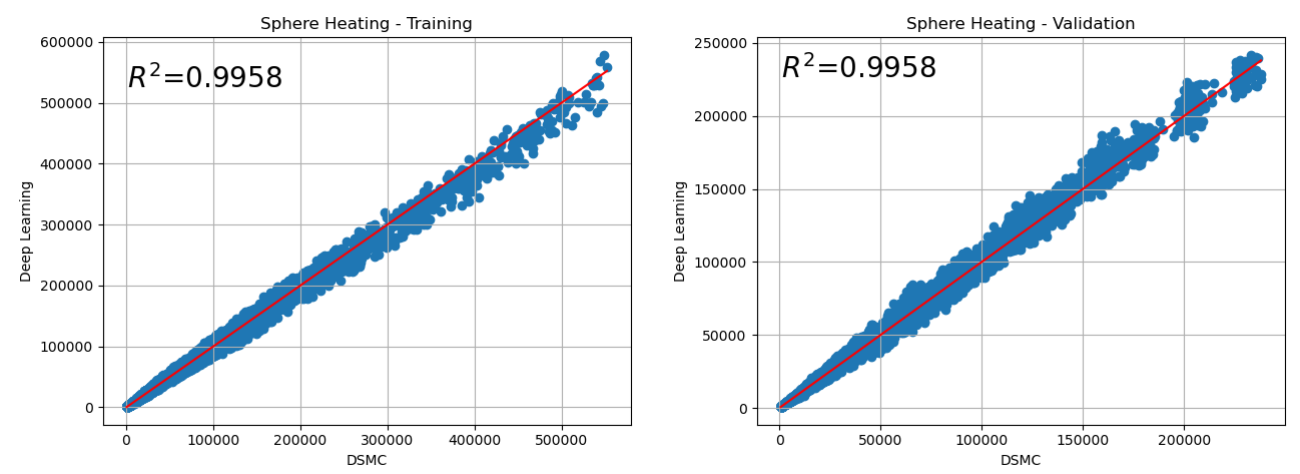

Figure 4.25: Sphere Heating Distribution
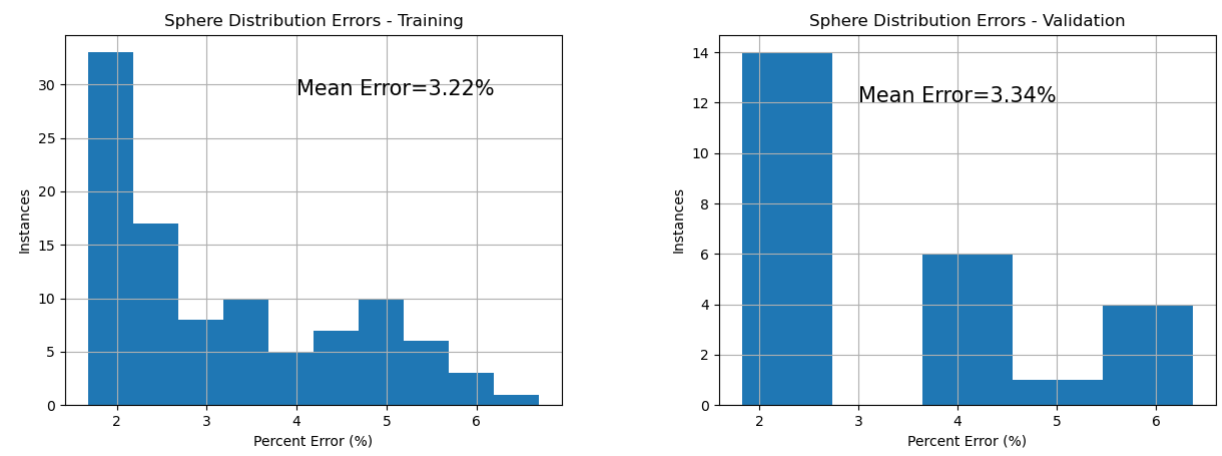

Figure 4.26: Sphere Error 

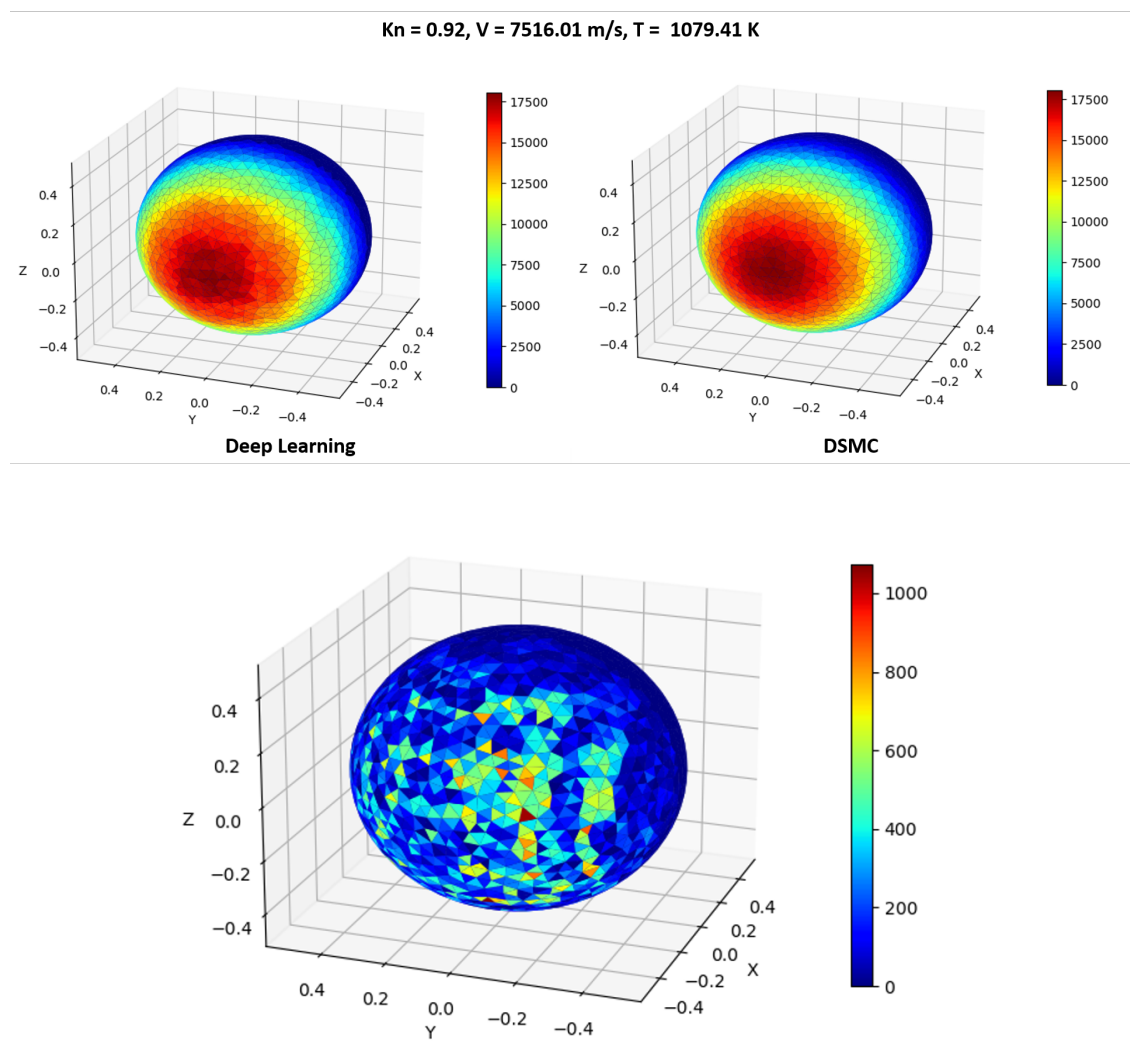

Difference

Figure 4.27: Sphere Low Heating Case

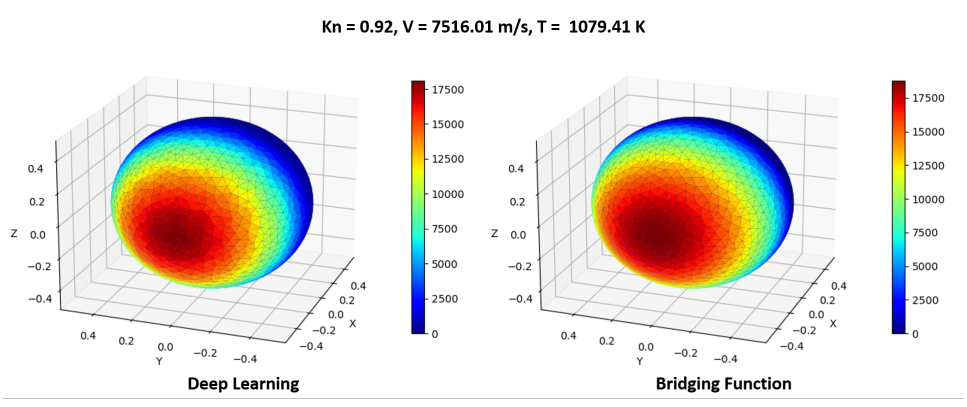

Figure 4.28: Sphere Bridging Function Comparison - Low Heating Case 

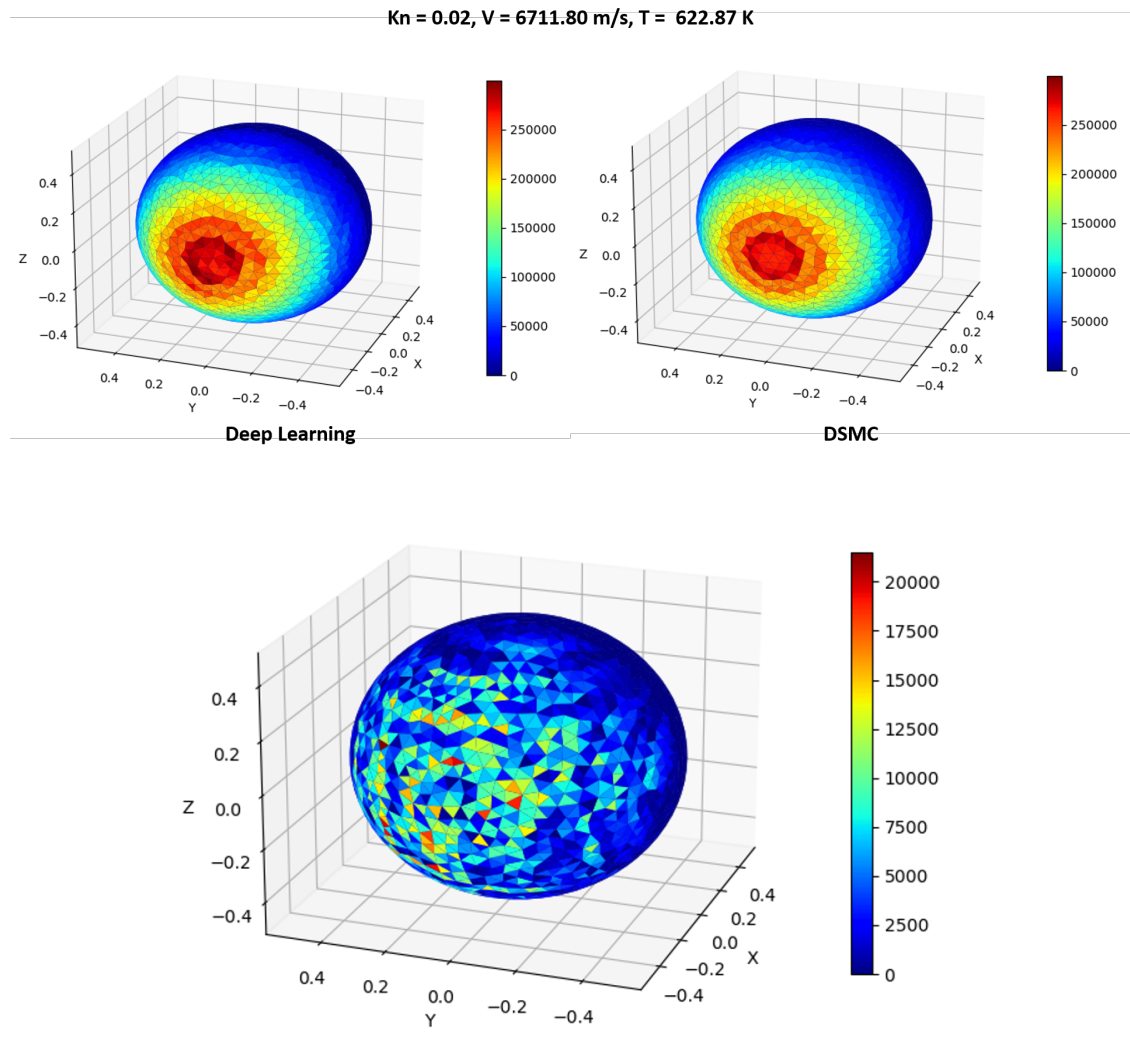

Difference

Figure 4.29: Sphere High Heating Case

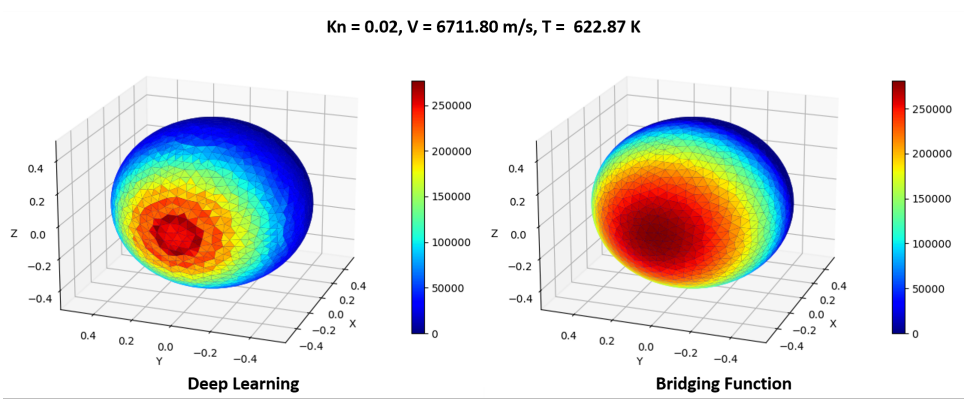

Figure 4.30: Sphere Bridging Function Comparison - High Heating Case 

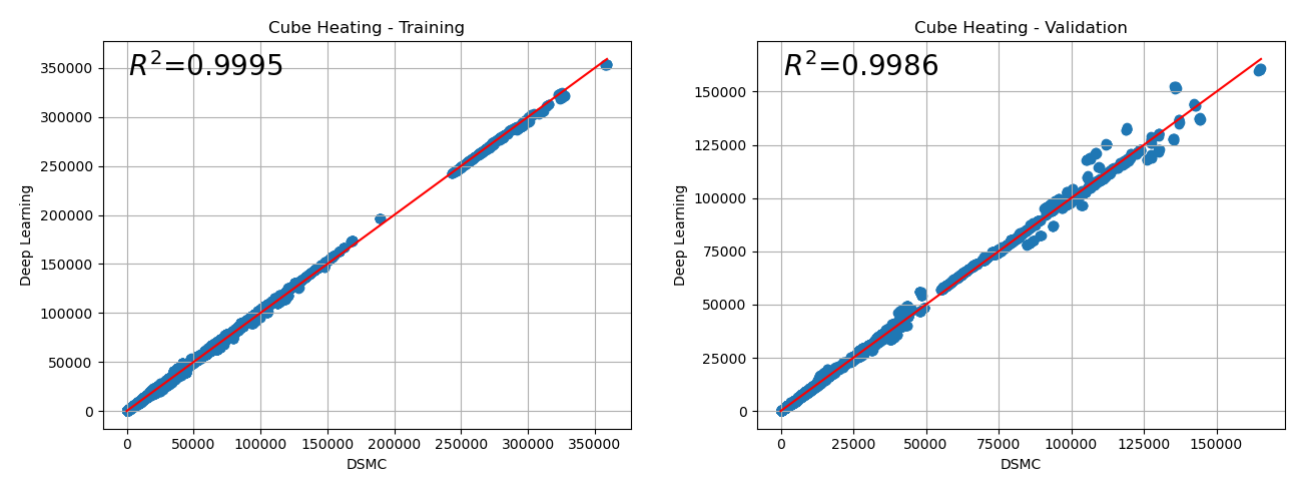

Figure 4.31: Cube Heating Distribution
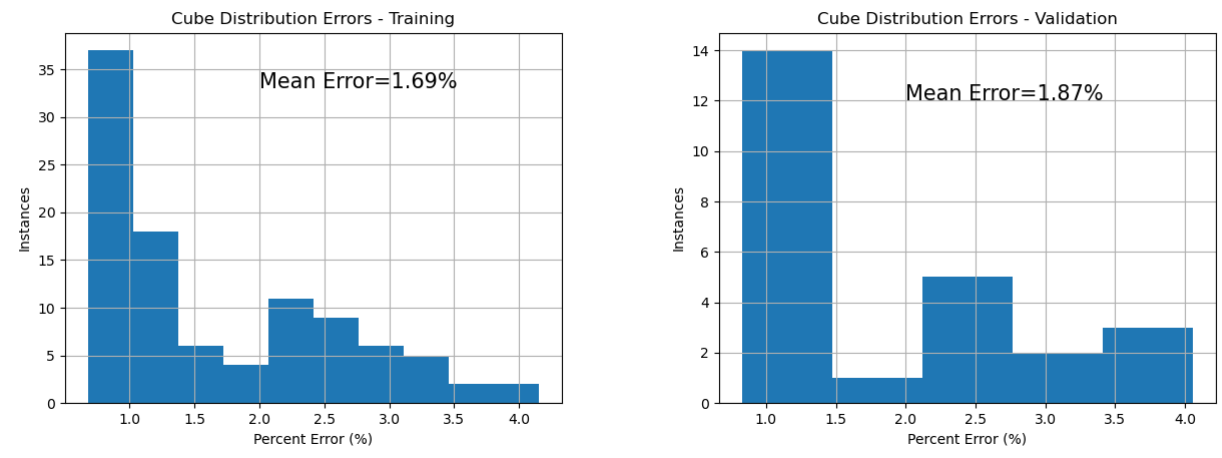

Figure 4.32: Cube Error 
$K n=0.92, V=7516.01 \mathrm{~m} / \mathrm{s}, T=1079.41 \mathrm{~K}$
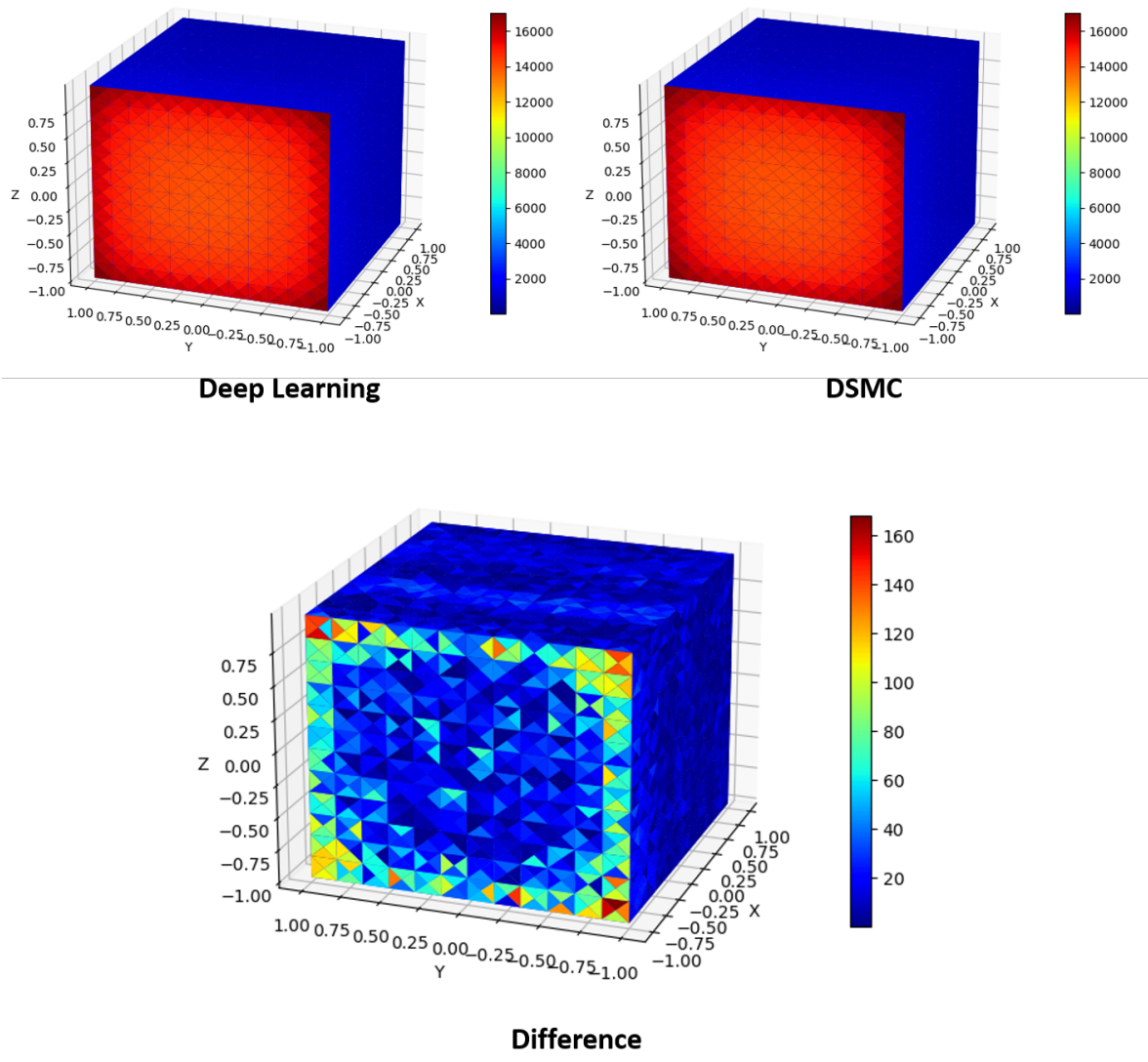

Figure 4.33: Cube Low Heating Case 

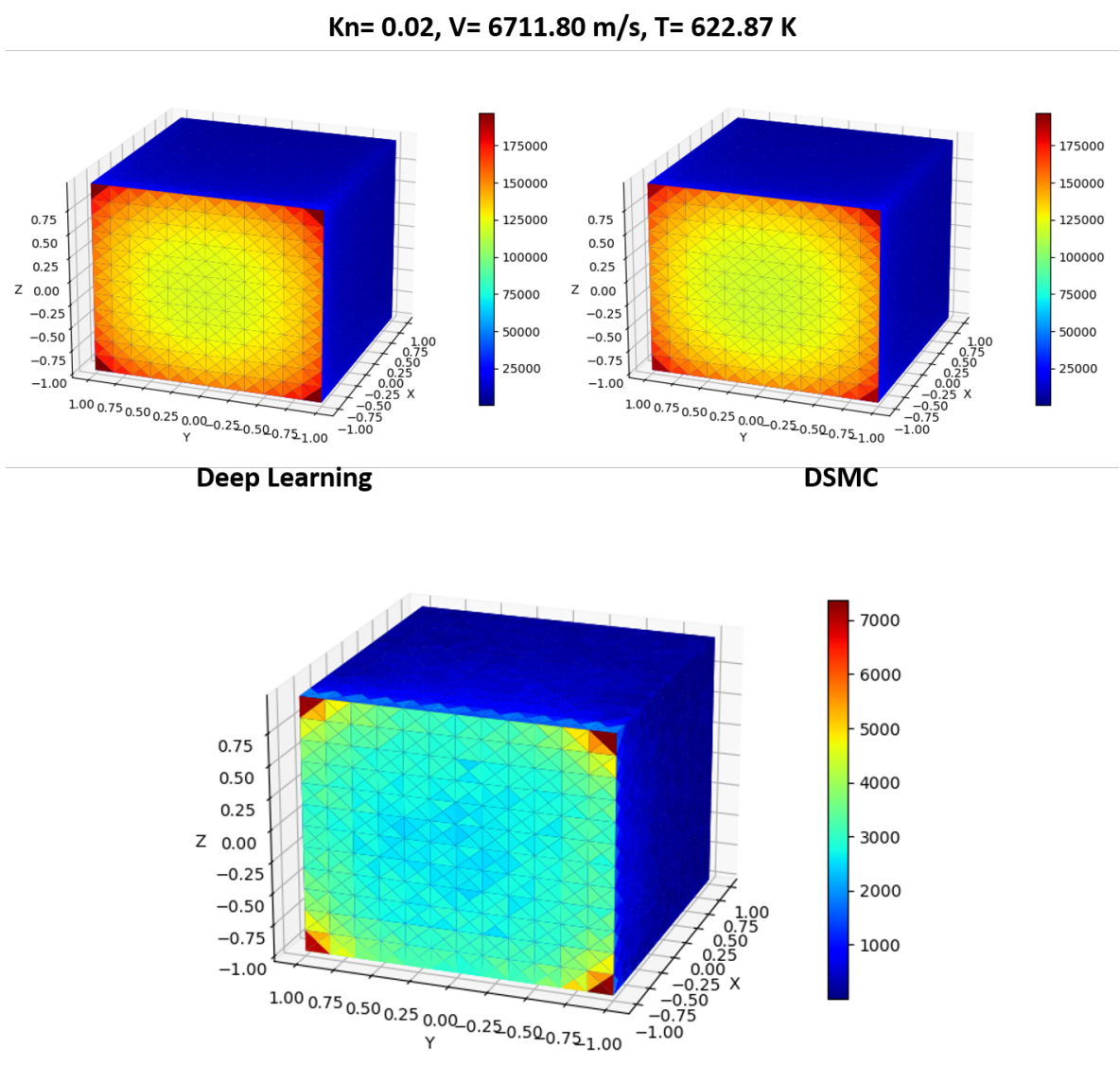

Difference

Figure 4.34: Cube Low Heating Case
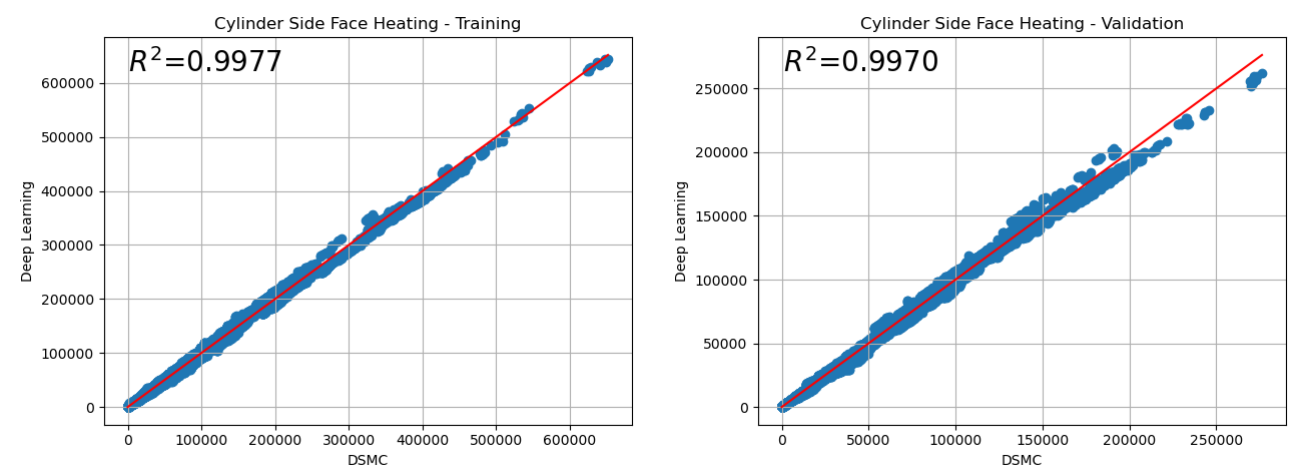

Figure 4.35: Cylinder Side Face Heating Distribution 

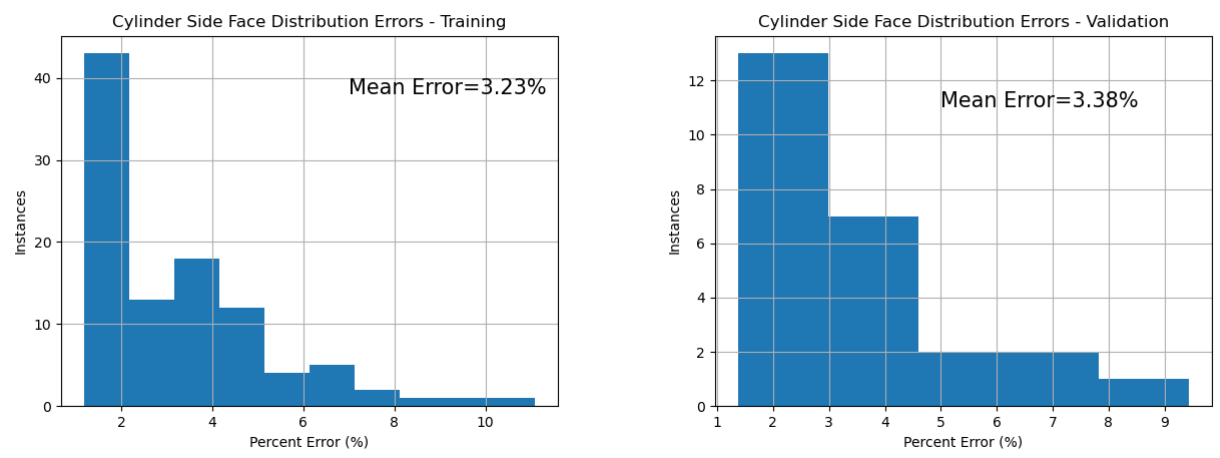

Figure 4.36: Cylinder Side Face Error
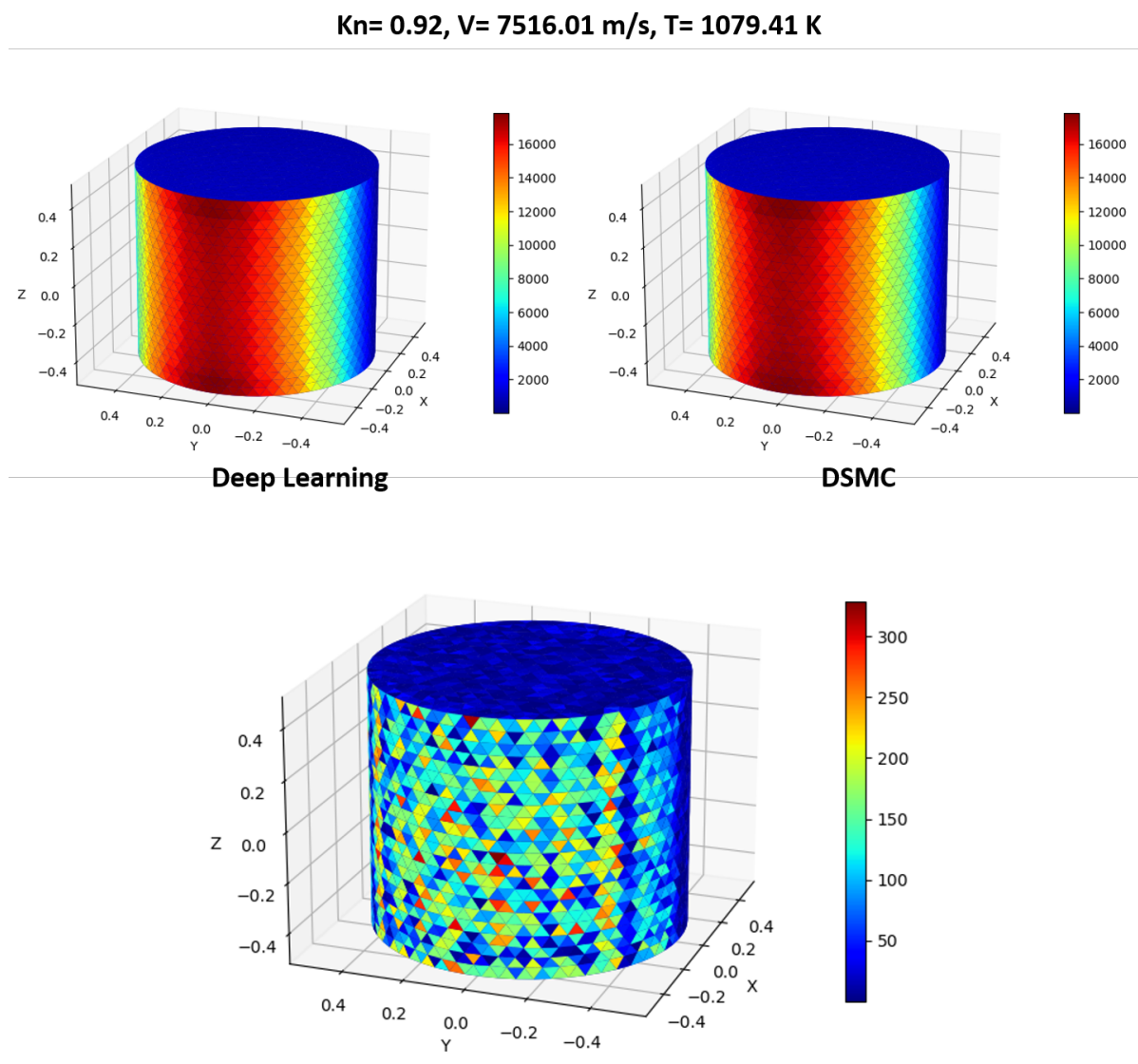

Difference

Figure 4.37: Cylinder Side Face Low Heating Case 

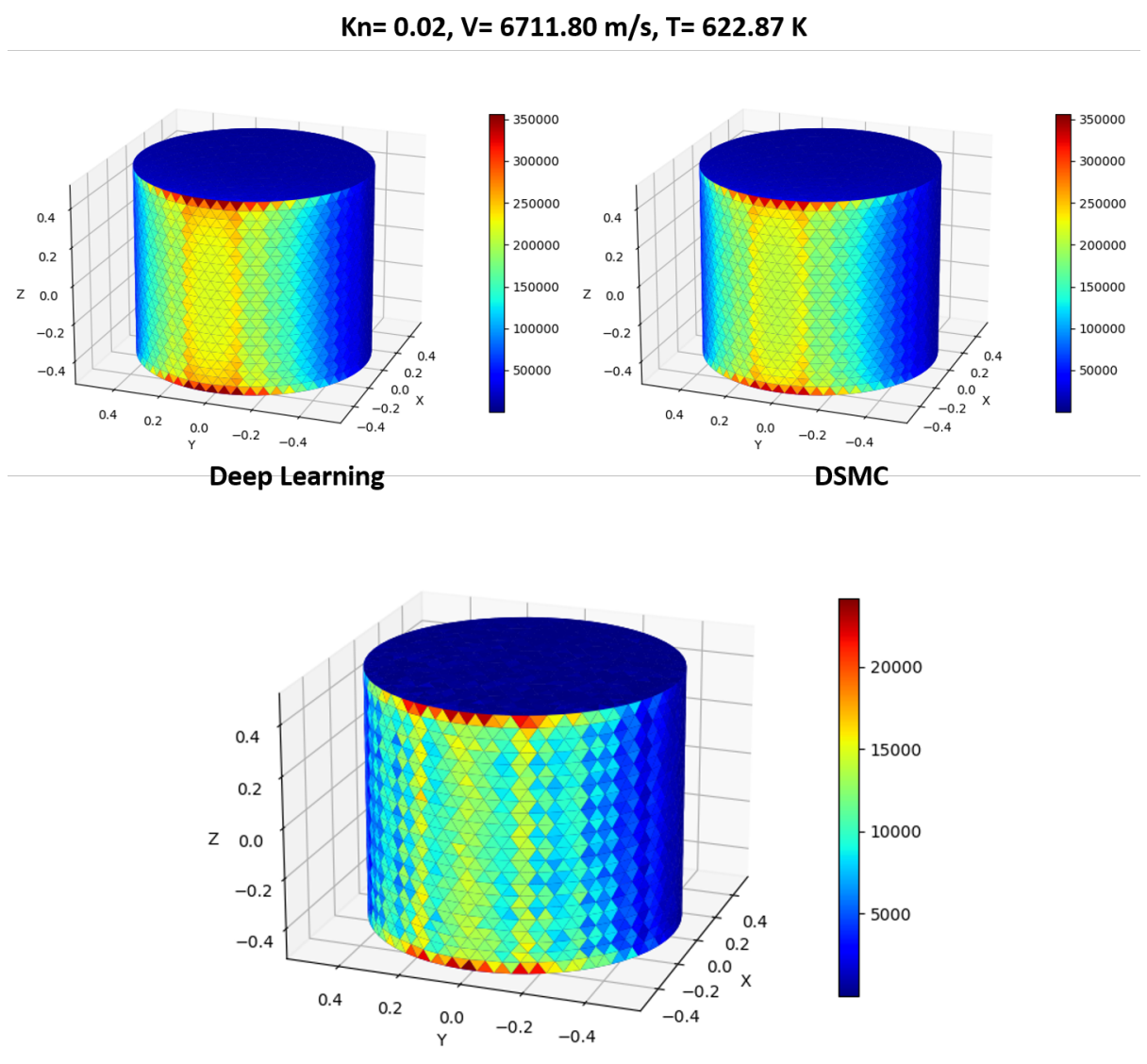

Difference

Figure 4.38: Cylinder Side Face High Heating Case
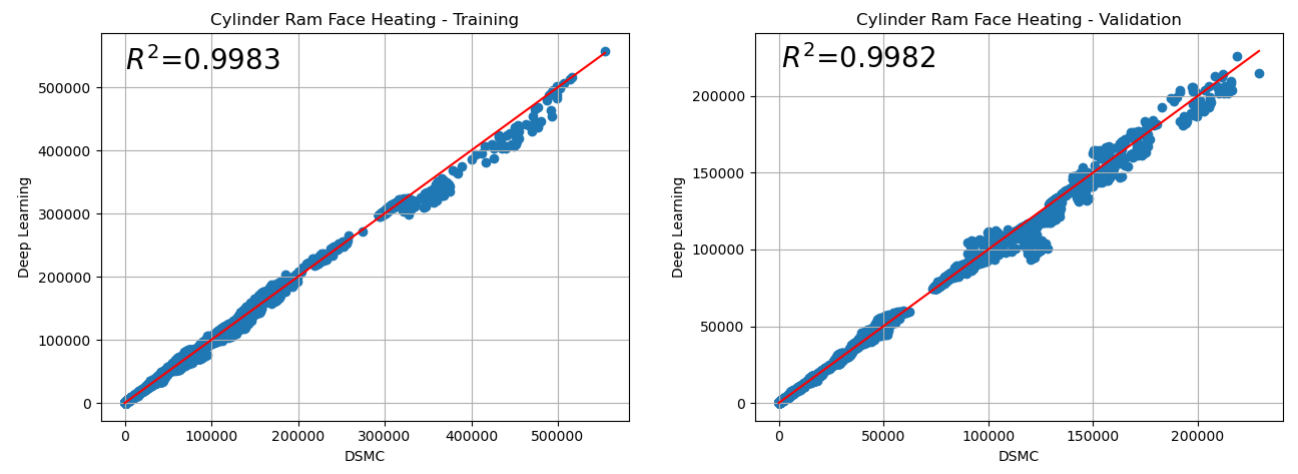

Figure 4.39: Cylinder Ram Face Heating Distribution 

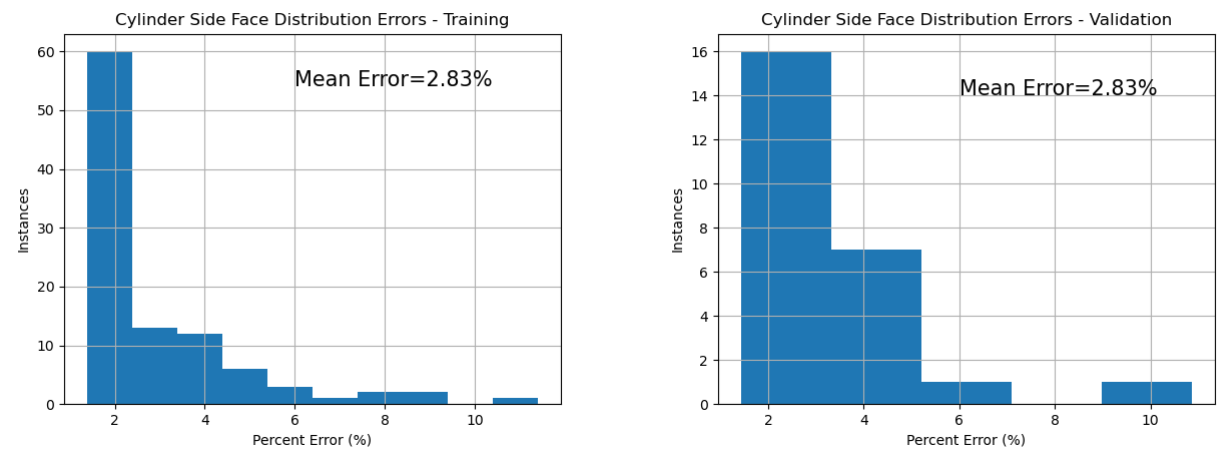

Figure 4.40: Cylinder Ram Face Error

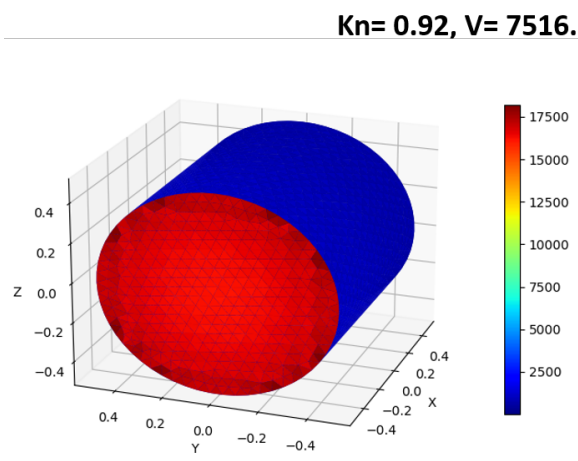

Deep Learning

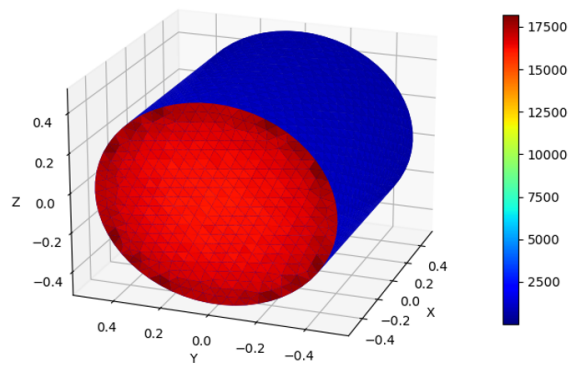

DSMC

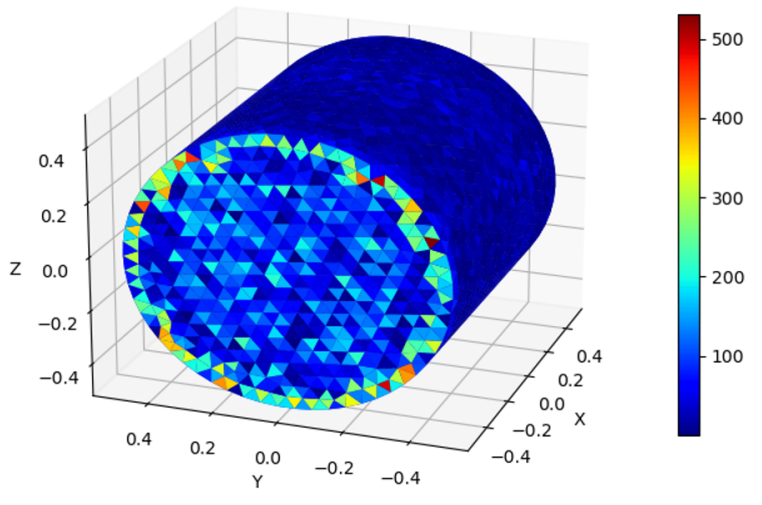

Difference

Figure 4.41: Cylinder Ram Face Low Heating Case 


\section{$K n=0.02, V=6711.80 \mathrm{~m} / \mathrm{s}, T=622.87 \mathrm{~K}$}
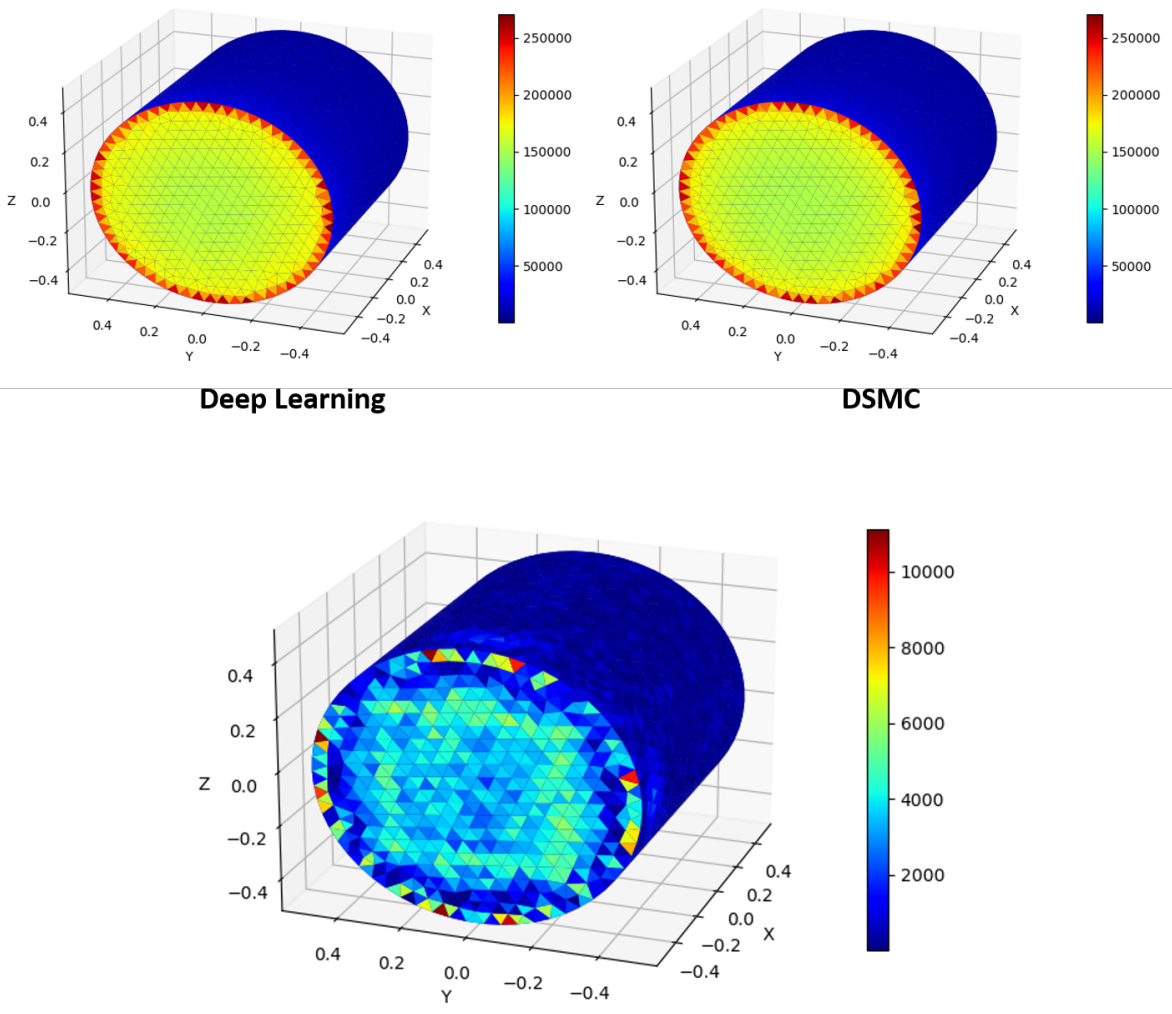

Difference

Figure 4.42: Cylinder Ram Face High Heating Case 


\subsection{Scaling Demonstration}

As previously stated, once the object is meshed, the distribution of mesh elements must not change. To allow the model to make valid predictions for objects of different characteristic lengths, the mesh can be scaled as long as the input Knudsen Number is scaled appropriately as well. Shown in Figure 4.43 is a demonstration of this ability, where the characteristic length is halved from its original value to one. The deep learning model, like the original cube validation cases, is still able to detect the areas of concentrated heating due to flow expansion. For the overall distribution, the model is able to replicate the DSMC data reasonably well. At lower, non-critical points of heating, there is slight over-prediction. Conversely, at higher values, there is slight underprediction, but the model still produces reasonable results.

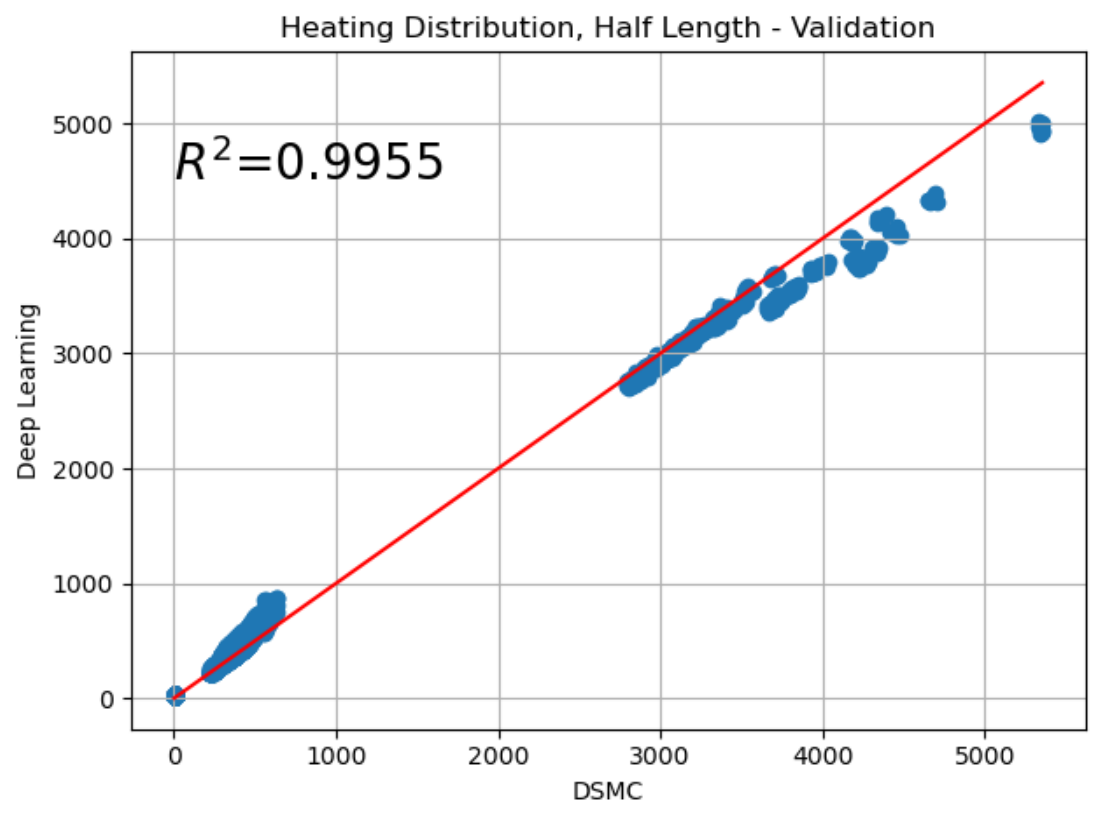

Figure 4.43: Scaling Heating 


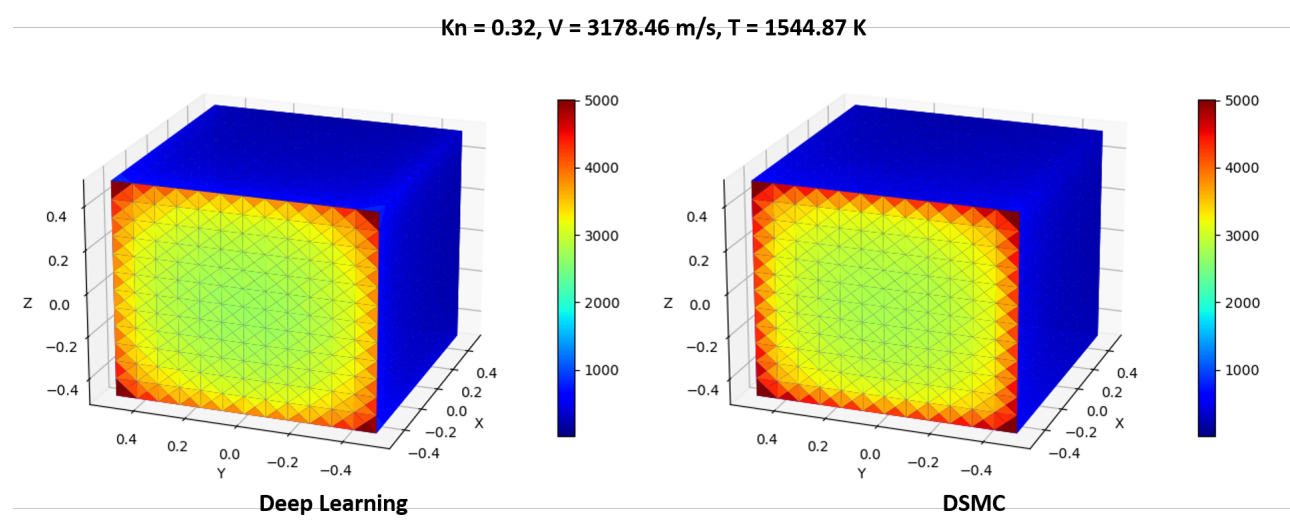

Figure 4.44: Scaling Heating Distribution

\subsection{Tumbling Object Demonstration}

Since objects in uncontrolled re-entry are usually tumbling or subject to attitude variations, a neural network was created to demonstrate the feasibility of modeling attitude variations. As seen in Figure 4.45, the performance of the model is reasonable, offering $R^{2}$ scores of over 0.98 for training and validation; however, when looking at the mean percentage error, the training dataset shows a decent percentage error with $6.82 \%$; however, the validation error is considerably higher with $14.99 \%$. The results are summarized below in Table 4.7 .

Table 4.7: Neural Network Performance - Attitude Variations

\begin{tabular}{lrc}
\hline & \% Error & $R^{2}$ \\
\hline Training & $6.82 \%$ & 0.9904 \\
Validation & $14.99 \%$ & 0.9835 \\
\hline
\end{tabular}

When visualizing the performance in Figures 4.47, 4.48, 4.49, and 4.50, it becomes apparent where the model excels and where it underperforms. It can be seen that cases with little attitude variations perform significantly better than those with high attitude variations. The underperformance may be attributed to the lack of adequate training samples to fully 
map the underlying function to include attitude variations. In the future, it is suggested that more simulations are completed to more adequately capture the entire input space.
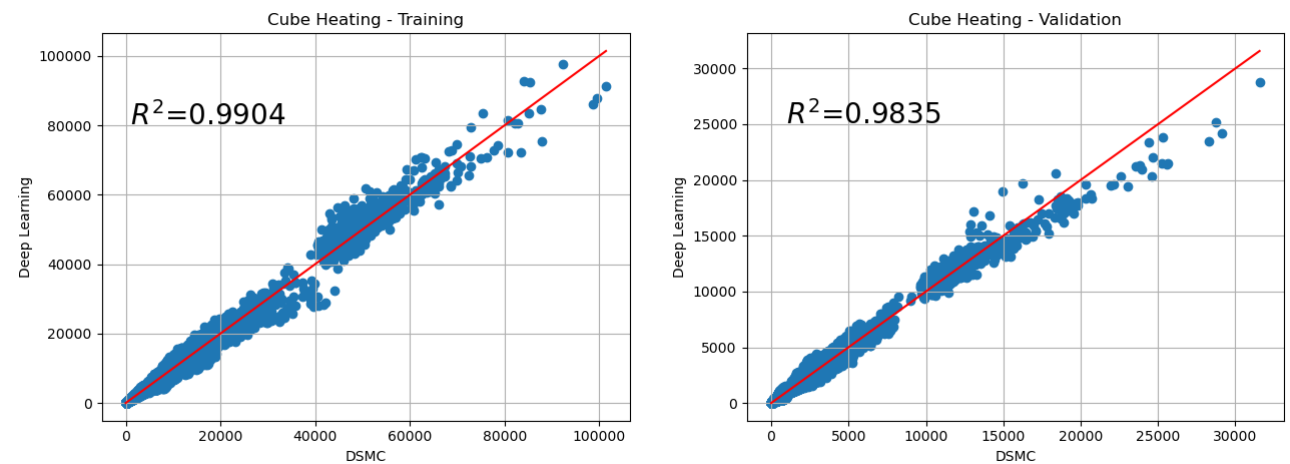

Figure 4.45: Cube Heating
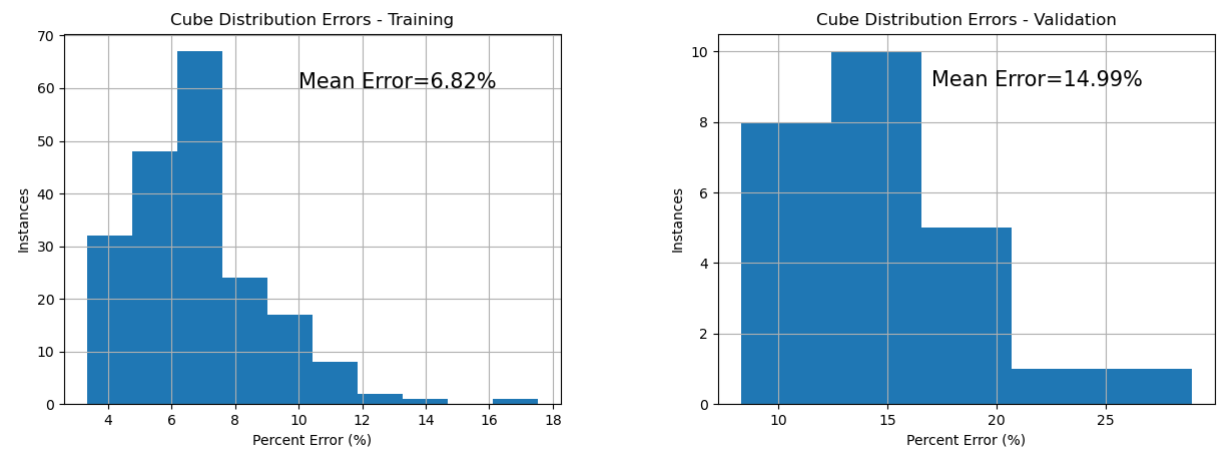

Figure 4.46: Cube Attitude Error 

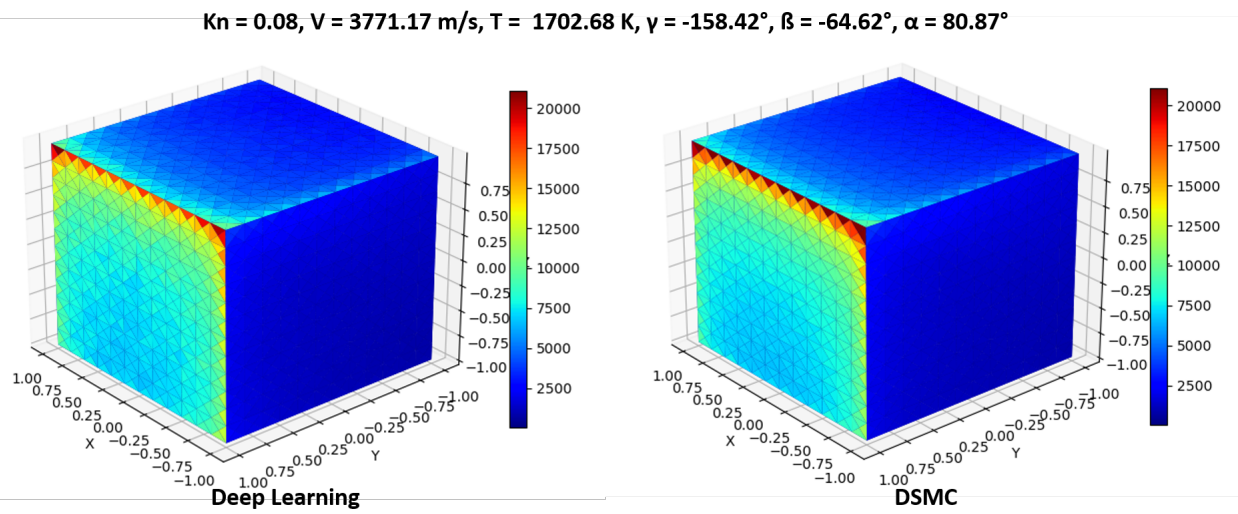

Figure 4.47: Best Training Case
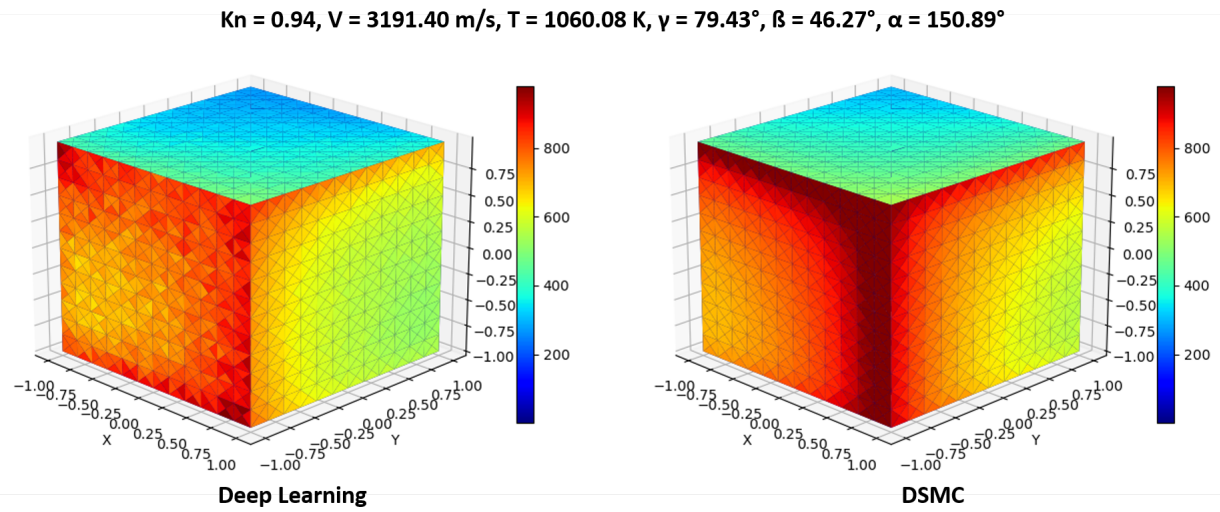

Figure 4.48: Worst Training Case

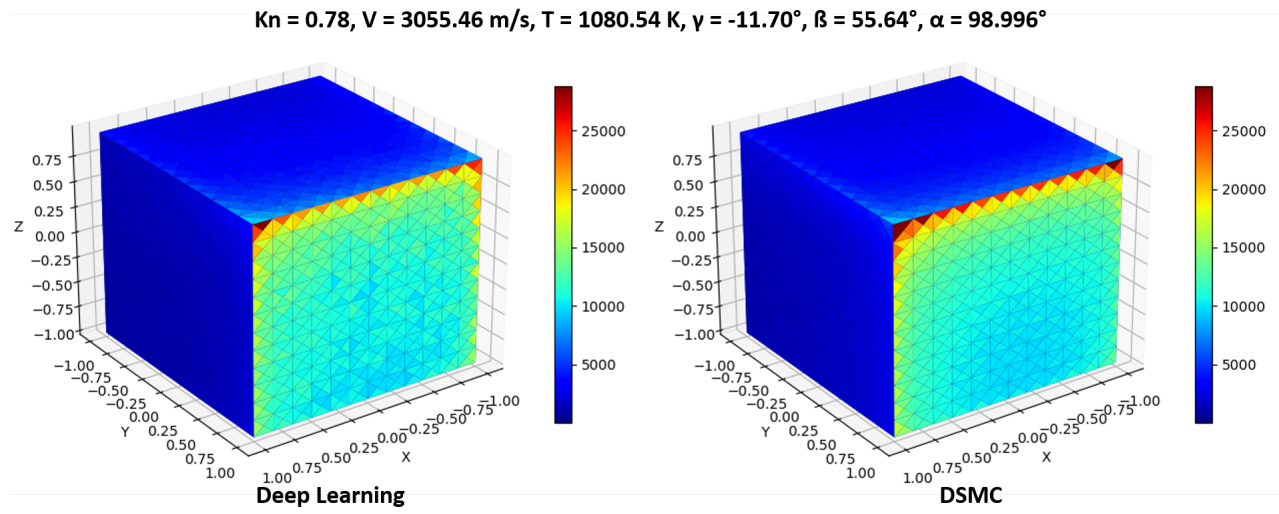

Figure 4.49: Best Validation Case 


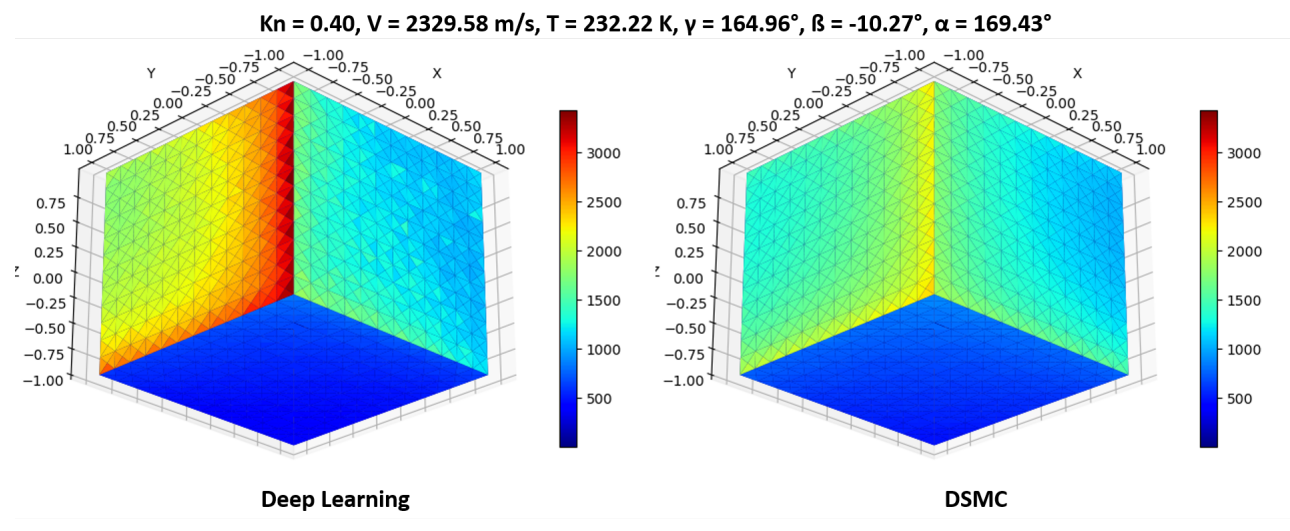

Figure 4.50: Worst Validation Case 


\section{Chapter 5}

\section{Summary, Conclusions, and Suggested Future Work}

The threat of polluting the area in LEO is significantly increased as the amount of objects in space is expected to dramatically rise over the next decade. With the increase of space assets, greater stress is placed on compliance of NASA predetermined policy that satellites and other spacecraft should re-enter Earth's atmosphere within 25 years of the end of its mission. As spacecraft or orbital debris re-enter Earth's atmosphere, risk assessment becomes the ultimate priority as to not cause any ground casualty. While fragments of spacecraft or other debris will demise due to the high heating experienced in the re-entry process, components that survive will hold significant amounts of kinetic energy as it impacts the ground which can cause serious damage to buildings and other assets.

To date, the most popular tool for re-entry risk assessment is object-oriented codes, where space debris and surviving spacecraft components are modeled as shape primitives. Analytical formulations are then used to conduct an aerothermodynamic analysis to determine if any fragments survive and where they may land. Object oriented tools are relatively inexpensive computationally, but do not offer the ability to model full spacecraft and are generally lower-fidelity compared to other tools. Furthermore, closed-form analytical formulations only exist for the free-molecular and continuum flow regimes for finite nose radius objects. For other shapes and the transition flow regime, bridging functions and shape fac- 
tors are used. Spacecraft oriented tools offer the ability to model the whole spacecraft as a composition of shape primitives. Here, the entire re-entry sequence can be modeled from initial entry into Earth's atmosphere to the breakup event and subsequent ground risk. While this is higher-fidelity, the computational cost also increases significantly. Numerical methods such as CFD (continuum flow regime) or DSMC (transition and free-molecular flow regime) offer the highest fidelity of all modeling tools, but also come at a significant computational expense. To create a tool that matches the fidelity of numerical methods but has the same level of computational demand as analytical models, machine learning is employed.

The feasibility of using machine learning to replicating full, high-fidelity heating distributions and drag coefficients on shape primitives in re-entry flow has been demonstrated. Using Gaussian Process Regression Modeling, the drag coefficient is accurately modeled, with a mean error across all shape primitives of under 1\%. For the full heating distribution, fully-connected neural networks are able to detect features such as concentrated values of high heating at the edges and corners for non-convex objects such as a cube and cylinder. For all shapes, there is a very good replication of DSMC data, thus demonstrating the ability of deep learning to replicate high-fidelity data in a fraction of a second. To extend the concept to shape primitives of all sizes and at different orientations, a scaling and attitude demonstration is shown. Future studies to further advance the development presented in this project include:

- Complete a sufficient amount of simulations to accurately capture attitude variations

- Implement pixelator from Computer Graphics for Space Debris [56]

- Uncertainty quantification for predictions

- Eventually extend concept to hollow shape primitives 
- Complete CFD Simulations for Continuum Flow Regime 


\section{Bibliography}

[1] NASA, (2019). Process for Limiting Orbital Debris

[2] "ARES | Orbital Debris Program Office | Debris Reentry | ORSAT." NASA, 2010, orbitaldebris.jsc.nasa.gov/reentry/orsat.html.

[3] Annaloro, J., Galera, S., Thiebaut, C., Spel, M., Van Hauwaert, P., Grossir, G., ... Omaly, P. (2020). Aerothermodynamics modelling of complex shapes in the DEBRISK atmospheric reentry tool: Methodology and validation. Acta Astronautica. doi:10.1016/j.actaastro.2020.03.00

[4] J. Gelhaus, C. Kebschull, V. Braun, N. Sanchez-Ortiz, E. Parilla Endrino, J. C. de Oliveira, andR. Dominguez-Gonzalez. Upgrade of ESA's Space Debris Mitigation Analysis Tool Suite. Technical Re-port ESA contract 4000104977/11/D/SR, European Space Agency, 2014

[5] Alessandro Falchi, Viola Renato, Edmondo Minisci, and Massimiliano Vasile. Fostrad: An advanced open sourcetool for re-entry analysis. In15th Reinventing Space Conference, 2017.

[6] Piyush M. Mehta, Edmondo Minisci, Massimiliano Vasile, Andrew Walker and Melrose Brown, "An Open-Source Hypersonic Aerodynamic and Aerothermodynamic Modeling Tool," 8th ESA symposium for Aerothermodynamics of Space Vehicles, Lisbon, Portugal, March 2-6, 2015.

[7] WU, Z., HU, R., QU, X., WANG, X., and WU, Z. (2011). Space Debris Reentry Analysis 
Methods and Tools. Chinese Journal of Aeronautics, 24(4), 387-395. doi:10.1016/s10009361(11)60046-0

[8] G. Koppenwallner, B. Fritsche, T. Lips, H. Klinkrad, SCARAB - A multidisciplinary code600for destruction analysis of space-craft during re-entry, in: Proceedings of the Fifth EuropeanSymposium on Aerothermodynamics for Space Vehicles, Cologne, Germany, 2005.

[9] J. Annaloro, P. Omaly, V. Rivola, M. Spel (2014) ELABORATION OF A NEW SPACECRAFT-ORIENTED TOOL: PAMPERO, European Symposium on Aerothermodynamics for Space Vehicles, Lisbon

[10] Y. Prévereaud, J.-L.Vérant,M.Balat-Pichelin, J.-M. Moschetta, (2015) Numerical and experimental study of the thermal degradation process during the atmospheric re-entry of a TiAl6V4 tank. Acta Astronautica, Vol. 122, pp. 258-286.

[11] Julien Annaloro, Stéphane Galera, P Karrang, Y Preveraud, J-L Verant, M Spel, P VanHauwaert, V Rivola, and P Omaly. Space Debris Atmospheric Entry Prediction wit hSpacecraft-Oriented Tools. In AeroThermoDynamics for Design for Demise (ATD3)Working Group, Noordwijk, The Netherlands, 2017.

[12] Piyush M. Mehta, Edmondo Minisci, Massimiliano Vasile, Andrew Walker and Melrose Brown, "Sensitivity Analysis towards Probabilistic Re-Entry Modeling of Spacecraft and Space Debris," AIAA Modeling and Simulation Technologies Conference, Aviation 2015, Dallas, Texas, June 22-26, 2015.

[13] Fay, J. A., and Riddell, F. R., Theory of Stagnation Point Heat Transfer in Dissociated Air, Journal of the Aeronautical Sciences, Vol. 25, No. 2, 1958, pp. 7385 
[14] Van Driest, E.R., The problem of aerodynamic heating, Aero. Eng. Rev., Vol. 15, No. 10, Oct 1956, pp. 26-41.

[15] S. J. Plimpton, S. G. Moore, et.al., (2019) Direct Simulation Monte Carlo on petaflop supercomputers and beyond Physics of Fluids, 31(086101)

[16] Bengt Sundén, Juan Fu, in Heat Transfer in Aerospace Applications, 2017

[17] Bastian E. Rapp, in Microfluidics: Modelling, Mechanics and Mathematics, 2017

[18] Cristina Parigini, Irene Pontijas Fuentes, Rodrigo Haya Ramos, and Stefania Cornara, "Debris tool and its use in mission analysis activities," in 8th ESA symposium on aerothermodynamics of space vehicles, Lisbon, Portugal, March 2015.

[19] Piyush M. Mehta, Andrew Walker, Earl Lawrence, Richard Linares, Dave Higdon and Josef Koller, "Modeling Satellite Drag Coefficients with Response Surfaces," Advances in Space Research, Vol. 54, No. 8, 2014, pp.1590-1607.

[20] Lips T, Fritsche B. A comparison of commonly used re-entry analysis tools. Acta Astronautica $2005 ; 57(2-8): 312-323$

[21] Klett R D. Drag coefficients and heating ratios for right circular cylinders in freemolecular and continuum flow from Mach 10 to 30. Sandia Corporation, SC-RR- 642141, 1964.

[22] P. Omaly, M. Spel, DEBRISK, a tool for Re-entry risk analysis, Proc. 5th IAASS Conf.Safer Space Safer World, Versailles, France, 2012, p. 70.

[23] Jouhaud, F.(2011).Mécanique du Vol Atmosphérique et Spatial: Recueil de modélisations, Rapport Technique NT4/17163DCSD, ONERA 
[24] Portelli, C., Salotti, L., Anselmo, L., Lips, T., and Tramutola, A. (2004). BeppoSAX equatorial uncontrolled re-entry. Advances in Space Research, 34(5), 1029-1037. doi:10.1016/j.asr.2003.11.011

[25] J.Annaloro, S.Galera, P.Kärräng, Y.Prévereaud, J.-L.Vérant, M.Spel, P.VanHauwaert, P.Omaly,Space debris atmospheric entry prediction with spacecraft-oriented tools, Conference Paper, 7th European Conference on Space Debris, Darmstadt (Germany), 2017.

[26] Schaaf, S.A., and Chambre, P.L., Flow of Rarefied Gases, High Speed Aerodynamics and Jet Propulsion, Princeton Univ. Press, Princeton, NJ, 1958, pp. 155.

[27] Detra, R. W., Kemp, N. H., and Riddell, F. R., Addendum to Heat Transfer to Satellite Vehicles Reentering the Atmosphere, Jet Propulsion, Vol. 27, No. 12, 1957, pp. 12561257.

[28] "Aerothermodynamics Course." NASA, Aerothermodynamics Course.

[29] Newton, I., Principia Motes Translation Revised, University of California Press, 1946.

[30] Legge, H., Hypersonic approximations for heat transfer and shear stress applied to continuum and rarefied plume impingement, DFVLR-IB 222-87 A23, 1987.

[31] Wilmoth, R. G., Mitcheltree, R. A., and Moss, J. N., Low Density Aerothermo dynamics of the Stardust Sample Return Capsule, Journal of Spacecraft and Rockets, Vol. 36, No. 3, 1999, pp. 436-441.

[32] Bird GA (1994) Molecular gas dynamics and the direct simulation of gas flows. Oxford University Press, Oxford

[33] Alexander F., Garcia A., (1997). The Direct Simulation Monte Carlo Method Computers in Physics, 11(588) 
[34] "Direct Simulation Monte Carlo (DSMC) Method." PIC, 2012, www.particleincell.com/2012/dsmc0.

[35] Saidi, M. S., et al. "Comparison between Lagrangian and Eulerian Approaches in Predicting Motion of Micron-Sized Particles in Laminar Flows." Atmospheric Environment, vol. 89, 2014, pp. 199-206. Crossref, doi:10.1016/j.atmosenv.2014.01.069.

[36] Denier, James P. "Anil W. Date: Introduction to Computational Fluid Dynamics." Theoretical and Computational Fluid Dynamics, vol. 23, no. 5, 2009, pp. 401-02. Crossref, doi:10.1007/s00162-009-0157-y.

[37] "DSMC Code Simulates Rarefied Gas Dynamic Environments." NASA, www.nasa.gov/centers/johnson/techtransfer/technology/ MSC-23445-1-dsmcdac.html.

[38] T. Elsken, J. Metzen, F. Hutter, (2019) Neural Architecture Search: A Survey. Journal of Machine Learning Research 20 (2019) 1-21

[39] Mohan, Aditya. "Bayesian Optimization and Hyperparameter Tuning - Towards Data Science." Medium, 15 Dec. 2020, towardsdatascience.com/bayesian-optimization-andhyperparameter-tuning-6a22f14cb9fa.

[40] Li, Lisha, and Kevin Jamieson. "Hyperband: A Novel Bandit-Based Approach to Hyperparameter Optimization." Journal of Machine Learning Research 18 (2018): 1-52.

[41] Keras-Team. "Bayesian Tuner Cannot Handle NaNs as Eval Results · Issue 509 · Keras-Team/Keras-Tuner.' GitHub, 2021, github.com/keras-team/kerastuner/issues/509.

[42] Tu, J., Liu,C., et.al., (2013) Practical Guidelines for CFD Simulation and Analysis Computational Fluid Dynamics 
[43] Helton, J., Davis, F., (2002). Latin Hypercube Sampling and the Propagation of Uncertainty in Analyses of Complex Systems.

[44] Olsson, A. et al. (2003). On Latin hypercube sampling for structural reliability analysis. Structural Safety: Volume 25, Issue 1, January, Pages 47-68. Retrieved January 5, 2018 from: https://doi.org/10.1016/S0167-4730(02)00039-5 Xin, L. (2014). Numerical Methods for Engineering Design and Optimization: Latin Hypercube Sampling (LHS)

[45] "MSIS-E-90 Atmosphere Model." MSIS-E-90 Atmosphere Model, 2010, ccmc.gsfc.nasa.gov/modelweb/models/msisvitmo.php.

[46] C. Geuzaine and J.-F. Remacle. Gmsh: a three-dimensional finite element mesh generator with built-in pre- and post-processing facilities. International Journal for Numerical Methods in Engineering 79(11), pp. 1309-1331, 2009.

[47] J.-F. Remacle, J. Lambrechts, B. Seny, E. Marchandise, A. Johnen and C. Geuzaine. Blossom-Quad: a non-uniform quadrilateral mesh generator using a minimum cost perfect matching algorithm. International Journal for Numerical Methods in Engineering 89, pp. 1102-1119, 2012.

[48] Sit, Hilarie. "Quick Start to Gaussian Process Regression - Towards Data Science." Medium, 12 June 2020, towardsdatascience.com/quick-start-to-gaussian-processregression-36d838810319.

[49] "Gaussian Processes — Scikit-Learn 0.24.1 Documentation." Scikit-Learn.Org, scikitlearn.org/stable/modules/gaussianprocess.html. Accessed 7 Aug. 2020.

[50] "Matern kernel — Scikit-Learn 0.24.1 Documentation." Scikit-Learn.Org, scikitlearn.org/stable/modules/generated/sklearn. gaussianprocess.kernels.Matern.html. Accessed 7 Aug. 2020. 
[51] "Sklearn.Gaussian-process.Kernels.WhiteKernel — Scikit-Learn 0.24.2 Documentation." Sci-Kit Learn, 2018, scikit-learn.org/stable/modules/generated/sklearn.gaussianprocess.kernels.WhiteKernel.html.

[52] "AutoML.Org." AutoML, www.automl.org. Accessed 8 Mar. 2021.

[53] "AutoKeras." AutoKeras, autokeras.com. Accessed 8 Mar. 2021.

[54] "Keras Tuner." Keras Tuner, keras-team.github.io/keras-tuner/documentation/. Accessed 7 Dec. 2020.

[55] "Tuners - Keras Tuner." Keras Tuner, keras-team.github.io/kerastuner/documentation/tuners. Accessed 7 Dec. 2020.

[56] Piyush M. Mehta, Gonzalo Blanco-Arnao, Davide Bonetti, Edmondo Minisci, Massimiliano Vasile, "Computer Graphics for Space Debris", 6th International Conference on Astrodynamics Tools and Techniques, Darmstadt, Germany, March 14-17, 2016.

$[57]$ 